\title{
Studying the temporal dynamics of the gut microbiota using metabolic stable isotope labeling and metaproteomics
}

\author{
by \\ Patrick Smyth \\ A Thesis Submitted in Partial Fulfillment \\ of the Requirements for the Master's of Science Degree of \\ Biochemistry with Specialization in Bioinformatics \\ Department of Biochemistry, Microbiology and Immunology \\ Faculty of Medicine \\ University of Ottawa \\ (C) Patrick Smyth, Ottawa, Canada, 2021
}




\begin{abstract}
The gut microbiome and its metabolic processes are dynamic systems. Surprisingly, our understanding of gut microbiome dynamics is limited. Here we report a metaproteomic workflow that involves protein stable isotope probing (protein-SIP) and identification/quantification of partially labeled peptides. We also developed a package, which we call MetaProfiler, that corrects for false identifications and performs phylogenetic and time series analysis for the study of microbiome dynamics. From the stool sample of five mice that were fed with ${ }^{15} \mathrm{~N}$ hydrolysate from Ralstonia eutropha, we identified 15,297 nonredundant unlabeled peptides of which 10,839 of their heavy counterparts were quantified. These peptides revealed incorporation profiles over time that were different between and within taxa, as well as between and within clusters of orthologous groups (COGs). Our study helps unravel the complex dynamics of protein synthesis and bacterial dynamics in the mouse microbiome.
\end{abstract}




\section{Acknowledgements}

I would like to thank Xu Zhang, Zhibin Ning, Janice Mayne, Mathieu Lavallée-Adam, and Daniel Figeys for providing critical feedback on my research. I would also like to give further thanks to Mathieu Lavallée-Adam for his supervision on writing the MetaProfiler software; Daniel Figeys for providing supervision on data analysis; Zhibin Ning for his help with the bioinformatic workflow; and Xu Zhang for designing the experimental protocol.

I would also like to thank my significant other, Jasmine Séguin for her love and support throughout my research work, as well for designing the MetaProfiler logo.

This work was supported by the Government of Canada through Genome Canada and the Ontario Genomics Institute (OGI-156), the Natural Sciences and Engineering Research Council of Canada (NSERC, grant no. 210034), and the Ontario Ministry of Economic Development and Innovation (ORF-DIG-14405), as well as a NSERC Discovery Grant to M. Lavallée-Adam and the stipends provided by TECHNOMISE and the Ontario Graduate Scholarship (OGS). 


\section{Statement of Contributions}

MetaProfiler was written by P. Smyth, with supervision from M. Lavallée-Adam and Z. Ning. The KNIME pipeline was developed by the OpenMS team, and modified by P. Smyth and Z. Ning. Data analysis was performed by P. Smyth with supervision from D. Figeys, Z. Ning, and X. Zhang. Experimental protocol was designed by X. Zhang. Mouse experiment, stool sample collection, protein extraction, trypsin digestion, and mass spec analysis were performed by X. Zhang, J. Mayne, J. I. Moore, and K. Walker. 


\section{List of Acronyms}

BH Benjamini-Hochberg

CCAC Canadian Council on Animal Care

CI Confidence Interval

CID Collision Induced Dissociation

COG Category of Orthologous Groups

DE Differential Evolutionary

DNA Deoxyibonucleic Acid

DTT Dithiothreitol

ESI Electrospray Ionization 
FFT Fast Fourier Transforms

FPR False Positive Rate

HCD Higher-energy collisional dissociation

HP Heavy Peptides

HPLC High-Pressure Liquid Chromatography

IAA Iodoacetamide

IBD Inflammatory Bowel Disorder

iTRAQ Isobaric Tags for Absolute and relative Quantification

KDE Kernel Density Estimation

LFDR Local False Discovery Rate

LFQ Label Free Quantification

LOOCV Leave One Out Cross Validation 
LR Labeling Ratio

MS Mass Spectrometry

NOI Naturally Occurring Isotopes

PEP Posterior Error Probability

PSM Peptide-Spectrum Match

RIA Relative Isotopic Abundance

RITZ Relative Intensity From Time Zero

RMSD Root Mean Square Deviation

RMSE Root Mean Square Error

RNA Ribonucleic Acid

RT Retention Time

SDS Sodium Dodecyl Sulfate 
SILAC Stable Isotope Labeling with Amino acids in Cell culture

SIP Stable Isotope Probing

TMT Tandem Mass Tags 


\section{Table of Contents}

List of Acronyms $\quad$ v

List of Figures $\quad$ xii

List of Tables $\quad$ xiv

1 Introduction $\quad 1$

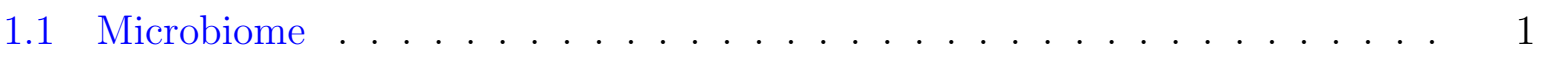

1.2 Gut Microbiome . . . . . . . . . . . . . . . . 2

1.3 Temporal Dynamics of Microbiome . . . . . . . . . . . . . . 9

1.4 Protein-Based Stable Isotope Probing . . . . . . . . . . . . . . . . . . 10

1.5 Available Isotopes for Protein-SIP . . . . . . . . . . . . . . . . . . . 12

1.6 Other Stable Isotope Probing Approaches . . . . . . . . . . . . . 16

1.7 Applications of Stable Isotope Probing . . . . . . . . . . . . . . . . 17

1.8 Challenges in Protein-SIP . . . . . . . . . . . . . . . 18

1.9 Local False Discovery Rate . . . . . . . . . . . . . . . . . . . 23

1.10 Project Aims . . . . . . . . . . . . . . . . . . . . . 24

2 Materials and Methods $\quad 25$

2.1 Mouse experiment and stool sample collection . . . . . . . . . . . . 25 
2.2 Mouse gut microbial protein extraction and trypsin digestion . . . . . . . 26

2.3 Mass Spectrometry Analysis . . . . . . . . . . . . . . . . 27

2.4 Extracting the Heavy Peptide Features from Protein-SIP Experiments . . . 30

2.5 Decomposition algorithm . . . . . . . . . . . . . . . 31

2.6 Filtering Spurious peaks . . . . . . . . . . . . . . . . . 32

2.7 Filtering Heavy Peptide Features using Local False Discovery Rate . . . . . 33

2.8 Data Imputation . . . . . . . . . . . . . . . . . . . . . 35

2.9 Data Analysis and Visualization . . . . . . . . . . . . . . 37

3 Results $\quad 40$

3.1 Taxonomic and functional characterization of the heavy labeled peptides in mouse gut microbiome . . . . . . . . . . . . . 58

3.2 Dynamics of ${ }^{15} \mathrm{~N}$ Incorporation into Peptides of the Mouse Gut Microbiota 64

$3.3{ }^{15} \mathrm{~N}$ incorporation rate differed according to microbial phylogeny . . . . . 72

3.4 Proteome Dynamics of Arabidopsis thaliana Seedling Roots . . . . . . . . . 75

$\begin{array}{llr}4 & \text { Discussion } & 90\end{array}$

$\begin{array}{llr}5 & \text { Conclusions } & 97\end{array}$

6 Future Work $\quad 98$

$\begin{array}{lr}\text { References } & 100\end{array}$

$\begin{array}{ll}\text { APPENDICES } & 113\end{array}$

A.1 Supplemental Figures . . . . . . . . . . . . . . . . . . . 113

A.1.1 Average taxonomic RIA and RITZ over time . . . . . . . . . . . 114

A.2 Supplemental Tables . . . . . . . . . . . . . . . . . . . . 124 
Curriculum Vitae

134 


\section{List of Figures}

1.1 An example of a typical metaprotemics experiment. . . . . . . . . . . 8

1.2 Example of how RIA and RITZ were calculated. . . . . . . . . . . . . . 14

1.3 An example of partial labeling in protein SIP experiments. . . . . . . . . . 20

2.1 Detailed Workflow. . . . . . . . . . . . . . . . . . 39

3.1 Bioinformatics Pipeline for Extracting Relative Isotopic Abundance and Relative Intensity from Time Zero. . . . . . . . . . . . . . . . . . 42

3.2 Overview of Experimental Workflow. . . . . . . . . . . . . . 44

3.3 The lower panels illustrates the Pearson correlation coefficient between corresponding samples. . . . . . . . . . . . . . . . . . 46

3.4 The overall retention time drift before and after alignment. . . . . . . . . . 49

3.5 The retention time drift before and after alignment for mouse 1 in day $43 . \quad 51$

3.6 Relative Intensity from Time Zero of Proteins in Mouse Gut Microbiome from Day 29 to $43 . \ldots \ldots$. . . . . . . . . . . . . . . . . 61

3.7 Functional Distribution of Heavy Labeled Proteins in Mouse Gut Microbiome from day 29 to $43 \ldots \ldots \ldots$. . . . . . . . . . . . 63

3.8 Heatmap before and after imputation. . . . . . . . . . . . . . 66

3.9 Isotope Incorporation Profile. . . . . . . . . . . . . . . . . . . 69

3.10 RIA and RITZ Distribution of Heavy Peptides Below 10\% LFDR Over Time. 71 
3.11 Taxon-Specific RIA and RITZ Profiles Over Time. . . . . . . . . . .

3.12 LR and RIA Distribution of Mixture, False, and True discoveries for the Soluble Fraction Sample. . . . . . . . . . . . . . . . . 78

3.13 LR and RIA Distribution of Mixture, False, and True discoveries for the Microsomal Fraction Sample. . . . . . . . . . . . . . . . 80

3.14 LR and RIA Distribution of Mixture, False, and True discoveries for the Organellar Fraction Sample. . . . . . . . . . . . . . . . . . . . 82

3.15 Isotope Incorporation Profile of Arabidopsis thaliana Seedling Roots. . . . 84

3.16 LR Profiles Over Time. . . . . . . . . . . . . . . . . . . 87

3.17 RITZ and RIA Distribution of Mixture, False, and True discoveries. . . . . 89 


\section{List of Tables}

3.1 Average Intensity of Peptides Found at Time Zero and Average Intensity of Peptides Found at Later Time Points. INT represents the average intensity of peptides found at time zero and peptides found at later time points . . . 53

3.2 Number of Peptides Found at Time Zero and Number of Peptides Found at Later Time Points . . . . . . . . . . . . . . . . . . . 55

3.3 The Average RIA of Peptides Found at Time Zero and Average RIA of Peptides Found at Later Time Points. The p-value is from a Wilcoxon test Correction was performed using BH procedure. . . . . . . . . . 57

A.1 Average taxonomic RITZ from day 29 to $43 \ldots \ldots \ldots \ldots$

A.2 Average taxonomic RITZ from day 29 to $43 \ldots \ldots \ldots$

A.3 Hygeometric tests for over-representation of taxa in RIA hierarchical clusters 132

A.4 Hygeometric tests for over-representation of taxa in RITZ hierarchical clusters133 


\section{Chapter 1}

\section{Introduction}

\subsection{Microbiome}

While there are several definitions given for microbiomes, in this manuscript, we will be using the definition provided by Berg et al. (2020), where they gathered 40 experts in diverse microbiome areas and hundreds more around the globe through online surveys to discuss current gaps in the frame of the European-funded MicrobiomeSupport project. The definition provided from this assembly is a modification from the first definition provided for microbiomes by Whipps et al. (1988).

The microbiome refers to the microorganisms (bacteria, eubacteria, fungi, virus etc.) living in a contiguous habitat, which has distinct physio-chemical properties. It also refers to the microbial, internal, and external structural elements compromising the microbiome, which brings about explicit ecological niches. The inhabitants are constantly interacting with one another creating a micro-ecosystem that changes in time and scale. This network 
is also integrated within a larger macro-ecosystem that is crucial for maintaining health and function within both systems.

Another term that is often used synonymously with microbiome is microbiota. However, this term refers only to the microorganisms that encompass the microbiome and it does not include the structural elements. Researchers will often look for core microbiota within similar environments to look for microorganisms that are crucial for maintaining the microbiome, such as keystone species.

\subsection{Gut Microbiome}

Much like other microbial communities, the human gut microbiome is an interactive and dynamic ecosystem that, when healthy, forms a symbiotic relationship with the host. The microbes are provided a nutrient rich environment and the host benefits from important physiological and metabolic functions, such as nutrient and mineral absorption; synthesis of enzymes, vitamins, amino acids, and short-chain fatty acids; and protection against pathogens, just to name a few(Rowland et al., 2018). The mucosal surfaces of the intestines provide the ecological niche needed for the gut microbiota to flourish.

Whereas composition can drastically change between individuals, core functionalities that are needed to establish a symbiotic relationship are conserved(Heintz-Buschart and Wilmes, 2018). When these functions are disturbed, serious health complications can arise, a state we call dysbiosis. Despite their level of importance, however, the influence of the microbiome on human health is largely understudied. Part of the reason for this is that research in this field is heavily driven by technological advancements. Fortunately, 
significant strides in genomic sequencing have been observed in recent years. Shafin et al. (2020) were able to sequence the entire genome of eleven human cohorts in just nine days, in contrast to the 13 years that it took for the human genome project to finish in 2003. With this technology, we can measure bacterial abundance and gene expression by counting the number of reads that were generated from their corresponding DNA or RNA sequence.(Conesa et al., 2016; McClure et al., 2013) We can then use this information to establish their functional roles within the gut microbiome. Genomic studies of gut microbial composition has increased our awareness of the impact of their alteration on human diseases, which include obesity(Maruvada et al., 2017), type II diabetes(Komaroff, 2017), inflammatory bowel disease (IBD)(Franzosa et al., 2019), cancer(Helmink et al., 2019), neurological disorders(Tremlett et al., 2017) and cardiovascular issues(Tang and Hazen, 2017)(see Figure 1.1), and has revealed that lifestyle, diet, and therapeutics can drastically change the composition and diversity of the gut microbiome(Shafin et al., 2020).

Many models and systems are available to study the gut microbiome(Starr et al., 2018), each providing advantages and limitations. Early research related to microbiome was limited by the fact that many microbiota could not be cultured in nutrient rich media. Today, however, there are techniques available that have been successful in culturing bacteria, which were previously thought to be unculturable(Lagier et al., 2018). In vitro monoculturing methods provide direct assessments of interactions between the host and microbome. In addition, since most of what we know about microorganisms is based on cultures, efforts are being made in uncovering the still remaining unknown species in the human gut. However, monoculturing methods cannot capture the complex interactions occur- 
ring with other neighbouring microbes. Another method is using ex vivo systems, such as gut on a chip(Kim et al., 2016), organoids(Sato and Clevers, 2013), continuous flow culturing(McDonald et al., 2015), RapidAIM(Li et al., 2020), etc. These are cost effective methods that can simulate the gut microbiome environment. However, these methods cannot be used to analyze microbiome flux as they cannot simulate the internal and external factors governing substrate break down. For this purpose, animal models provide an alternative method for analyzing microbiome. Rodent models are a common choice as they have key similarities with human gut microbiota at the phylum and family levels, as well as sharing 99\% genomic similarity(Starr et al., 2018). The disadvantage of using animal models are the cost, facilities, and time needed to accommodate them.

While useful, the bulk of microbiome research is based on genomic sequencing and there is a pressing need to move beyond studying compositional changes. The genetic material cannot tell which pathways are active. It also cannot differentiate between dead, active, and dormant microbes as the presence of a gene does not mean that it is expressed. Researchers have alternatively used transcriptomics to assess the expressed genes of the gut microbiome(Lavelle and Sokol, 2018). The move from metagenomics to metatranscriptomics has been fairly straightforward as many of the software used for metagenomics are applicable to metatranscriptomics as well. In fact, the combination of these two techniques have been shown to improve gene prediction and genome assembly(Heintz-Buschart et al., 2016). More recently, metaproteomics, which uses tandem mass spectrometry (MS/MS) to identify and quantify proteins, has also been used to assess the expressed proteins of the gut microbiome(Zhang et al., 2018b). Compared to metagenomics and metatranscriptomics, 
metaproteomics provides a deeper insight into the functionality of the gut microbiome as not all genes and transcripts are translated into proteins. However, the use metaproteomics in microbiome research has not been as straightforward since early research was encumbered by the lack of bioinformatics tools capable of analyzing mass spectrometry data from microbiomes. Fortunately, several software packages have recently emerged to address this issue, such as MetaLab(Cheng et al., 2017), MetaProteomeAnalyzer(Muth et al., 2018), Galaxy-P(Jagtap et al., 2015), trans-proteomic pipeline(Pedrioli, 2010), and OpenMS(Röst et al., 2016). These platforms provide tools that can be easily integrated into a pipeline for pre-processing and post-processing mass spectrometry data. By studying the proteome in addition to the genome, it allows to get a clearer picture of which proteins are behind key processes and to determine the active microbes and their functional roles within the gut microbiome. Moreover, the dynamicity of the microbiome also needs to be characterized as it is an important determinant of the structure of the microbiota. Metaproteomics in Microbiome Research

Metaproteomics involves analyzing proteins from microbial communities. By studying the microbes in their natural environment, we can capture the complex interactions occurring between host and microbes. The typical procedures for proteomics involve bacterial harvesting, protein extraction, denaturation, and digestion, followed by liquid chromatography (separation). The peptides is then separated by High Pressure Liquid Chromatography (HPLC), ionized by Electrospray Ionization (ESI), and analyzed by MS/MS. As peptides elute from the HPLC column, the mass spectrometer continuously cycles between ion selections and ion fragmentation. The purpose of the cycle is to select ions that are 
coming from peptides and to identify them via their fragmentation pattern. This is because many of the charged molecules will be the result of contaminants and obtaining the mass of the peptide alone is not sufficient to identify its sequence. Briefly, In the first part of the cycle, the mass spectrometer detects the ionized tryptic peptides by their mass-tocharge ratio $(\mathrm{m} / \mathrm{z})$, which are represented by a mass spectrum called MS1 or simply MS. Next, a series of ions are selected based on their intensity (i.e. current generated from the ions) for further sequential analysis. Each ion is isolated by the mass spectrometer and fragmented into smaller pieces, which is commonly done using Collision Induced Dissociation (CID)(Sleno and Volmer, 2004) or Higher-energy C-trap dissociation (HCD)(Olsen et al., 2007). The fragments are then detected by the mass spectrometer, resulting in the MS2 spectrum or MS/MS spectrum. To identify the MS/MS spectra, we can perform a database search, wherein spectra are compared against theoretical patterns generated from known sequences. The list of peptides available for matching are generated from in silico digestion of a protein database. Peptides associated with an MS2 spectrum are often called peptide-spectrum matches (PSMs). There are two main strategies for scoring PSMs: deterministic and probabilistic models. Software tools like SEQUEST(Eng et al., 1994) uses a deterministic approach where they calculate a cross-correlation score between the observed and theoretical spectra. The main drawback of this approach is that the cross-correlation scores are difficult to interpret and takes a lot of computational resources to calculate. However, Fast-Fourier Transforms (FFT) can be used to accelerate computation time. It is also heavily affected by the quality of the spectra as well as the length of the peptide, selected modifications, and charge state.(Verheggen et al., 2020) Mascot(Perkins et al., 1999) and Andromeda(Cox et al., 2011), on the other hand, use a 


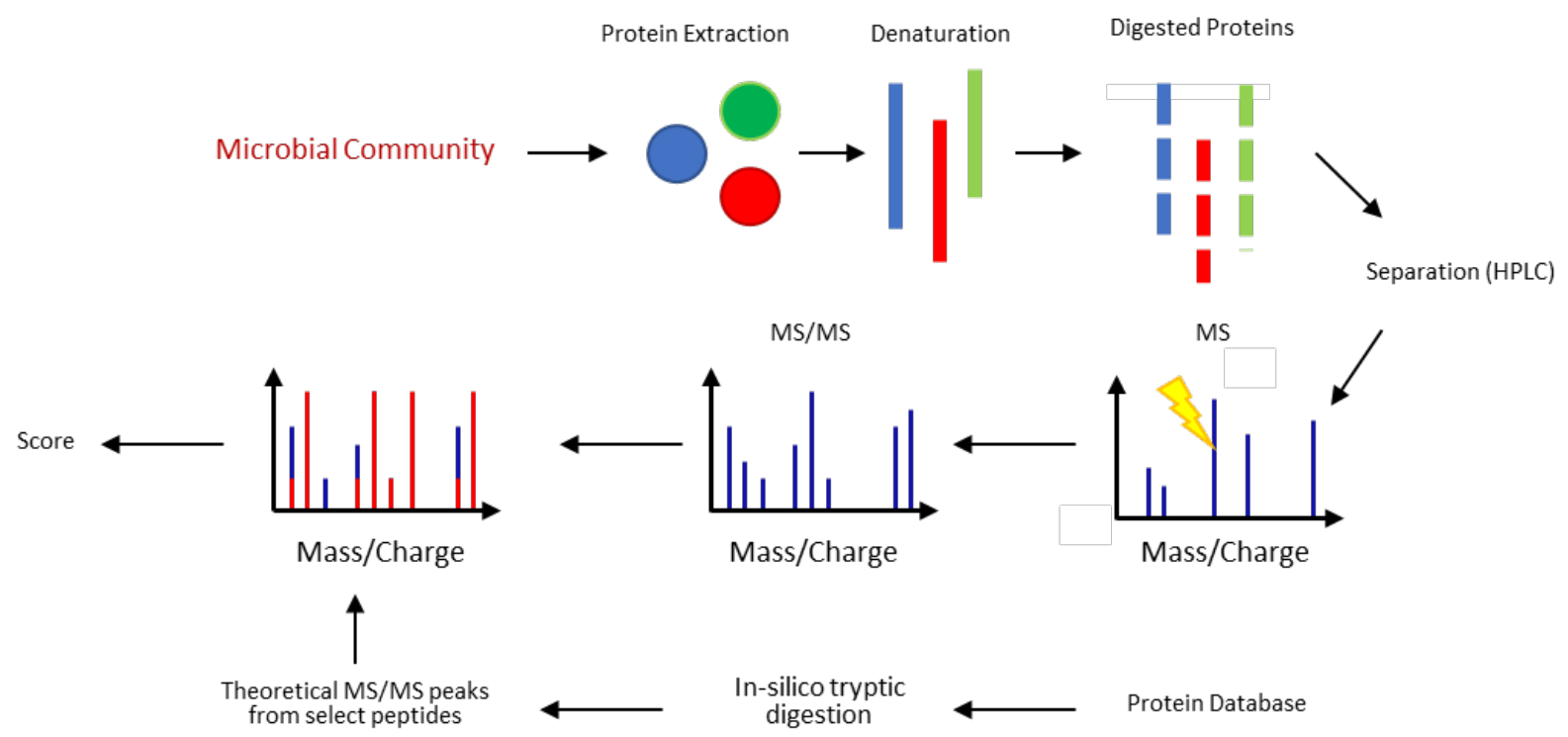


Figure 1.1: An example of a typical metaprotemics experiment. The steps follow: bacterial harvesting, protein extraction, denaturation, and digestion, liquid chromatography, and mass spectrometry. The circle represents the folded protein, the line represents the denatured protein, and the dashed line represents the digested protein. Database search is then used to identify the peptides in the sample. 
probabilistic model. In this strategy, the algorithm calculates the probability of the PSM being from a false discovery by using decoys that were assigned with peptide ids. The advantage of this approach is that it is easier to interpret than the deterministic approach. However, in contrast, the probabilistic model suffers from reproducibility and is heavily influenced by the size of the search space.(Verheggen et al., 2020) As the database size grows, the distribution of false discoveries widens and the difference in score between false and true discoveries decreases(Verheggen et al., 2020). Thus, this results in fewer number of identified peptides. To decrease the search space, our lab recently developed an approach, as part of the package suite MetaLab, that generates a sample specific database by using delegate spectra to search against the large protein database and then extracting the protein sequence according to the resulting peptide identification list to construct the sample-specific database(Xiao et al., 2015).

\subsection{Temporal Dynamics of Microbiome}

Most disease association studies on gut microbiome have focused on microbiome compo-

sition. However, there have been studies suggesting that microbiome dynamics can be a feature in clinical outcome(Gajer et al., 2012; Livanos et al., 2016). Gajer et al. showed that microbial fluctuation in vaginal bacterial communities was different in women with bacterial vaginosis(Gajer et al., 2012). Another study showed that mice administered periodically with antibiotics showed a higher rate of change in microbiota and an increased susceptibility to type 1 diabetes.(Livanos et al., 2016)

The first dense time-series analysis of the human microbiome was conducted by Capo- 
raso et al. (2011), where they monitored two individuals, one for six months and the other for fifteen, from four body sites over 396 timepoints. The longitudinal study demonstrated that only $5 \%$ of the microbial species were observed in 130 samples in the gut and oral microbiome, representing the core microbiota. Another study monitored the skin, gut, and oral microbiome of 85 individuals between the ages of 17 to 21 on a weekly basis for a period of three months(Flores et al., 2014). While some individuals saw no changes in microbial composition, others saw rapid fluctuations. Interestingly, the use of antibiotics during and before the experiment did not affect the rate of change in microbiome composition. Despite these studies showing differences in the rate of compositional changes, each still retained a personalized microbiome. These finding emphasizes the need to characterize the temporal dynamics of the gut microbiome as both microbial composition and their associated rate of change represent personalized features of human microbiome. By understanding the properties behind microbiome dynamics and the causes behind structural changes in microbiota, we would be able provide further insights into the causal relationships between species and disease. It would also have clinical implications as the rate of recovery of patients undergoing procedure that affect the gut microbiome could be improved.

\subsection{Protein-Based Stable Isotope Probing}

While metaproteomics is a powerful method in determining the functional repertoire of the gut microbiome, it cannot assess the activity of the microbes since old and newly translated proteins cannot be differentiated. To address this, metabolic incorporation of

heavy stable isotopes (usually ${ }^{13} \mathrm{C}$ or ${ }^{15} \mathrm{~N}$ ) can be used to track the newly synthesized 
biomolecules(Jehmlich et al., 2016). By using stable isotopes, microorganisms that assimilate these isotopes can be determined. In addition, by quantifying the proteins that were derived from the labeled substrate, rate of synthesis and, by using phylogenetic information, activity of the microbe can be determined.

Two approaches are currently available for metabolic protein labelling. One uses substrates or nutrients such as labeled glucose, ammonium, or protein hydrolysates, which become incorporated into amino acids, a technique called Stable Isotope Probing (SIP). The second uses a growth medium that contains specifically labelled amino acids (usually lysine and or arginine), which is referred as Stable Isotope Labeling with Amino acids in Cell culture (SILAC). Since the isotopes used are heavier than their most abundant version, a shift in mass will be observed by mass spectrometry, which can be used to detect their incorporation into substrate-derived biomolecules. SIP has become an appealing approach, since two important parameters can be extracted: the average proportion of isotope incorporated in a peptide of interest, termed relative isotopic abundance (RIA), and the relative ratio between the light peptide and the estimated intensity of the heavy peptide, which is termed labeling ratio (LR). LR measures the proportion of proteins that are produced using the isotopes from diet. By monitoring this value over time, the rate of newly synthesized proteins can be evaluated. Labeling ratio is calculated using equation 1.1

$$
\mathrm{LR}=\frac{I_{h}^{p}}{I_{l}^{p}+I_{h}^{p}},
$$

where $I_{l}^{p}$ is the sum of the intensities of the light peaks for peptide $p$ in the spectra and $I_{h}^{p}$ is the sum of the intensities of the heavy peaks for $p$ in the spectra. 
On the other hand, RIA measures the elemental flux of the isotope. By characterizing the functional and taxonomic origin of the peptide, it gives insight on how the stable isotopic substrate is being converted into biomass and which taxa assimilates this substrate. Thus, measuring RIA can predict which taxa this substrate is limited to. RIA is calculated using equation 1.2

$$
\mathrm{RIA}=\frac{H^{p}-M^{p}}{F^{p}-M^{p}},
$$

where $H$ is the $\mathrm{m} / \mathrm{z}$ position at the center of the theoretical isotopic distribution of the heavyp, $M$ is the monoisotopic peak of the light $p$, and $F$ is the $\mathrm{m} / \mathrm{z}$ position of the fully labeled peptide $p$. An example of how RIA and LR is calculated is demonstrated in Figure 1.2.

This study proposes using stable isotopes combined with proteomics to provide a deeper understanding of the mechanistic processes governing microbiome dynamics using the properties behind the assimilation of labeled substrates by the gut microbiota.

\subsection{Available Isotopes for Protein-SIP}

As shown from the average composition for amino acids, $\mathrm{C}_{4.7} \mathrm{H}_{7.8} \mathrm{~N}_{1.4} \mathrm{O}_{1.5} \mathrm{~S}_{0.04}$ (also known as an averagine)(Senko et al., 1995), hydrogen is the most abundant element in proteins. However, the stable isotope, deuterium $(2 \mathrm{H})$, has not been used in SIP as there is a high degree of hydrogen exchange with the aqueous medium. This is especially true for hydrogen atoms in amide groups and protein side chains. Thus, since exchange will occur during sample preparation, the task of tracking the microorganism that assimilated the 
AADDAAGLTISEK in Sample 4 on Day 4

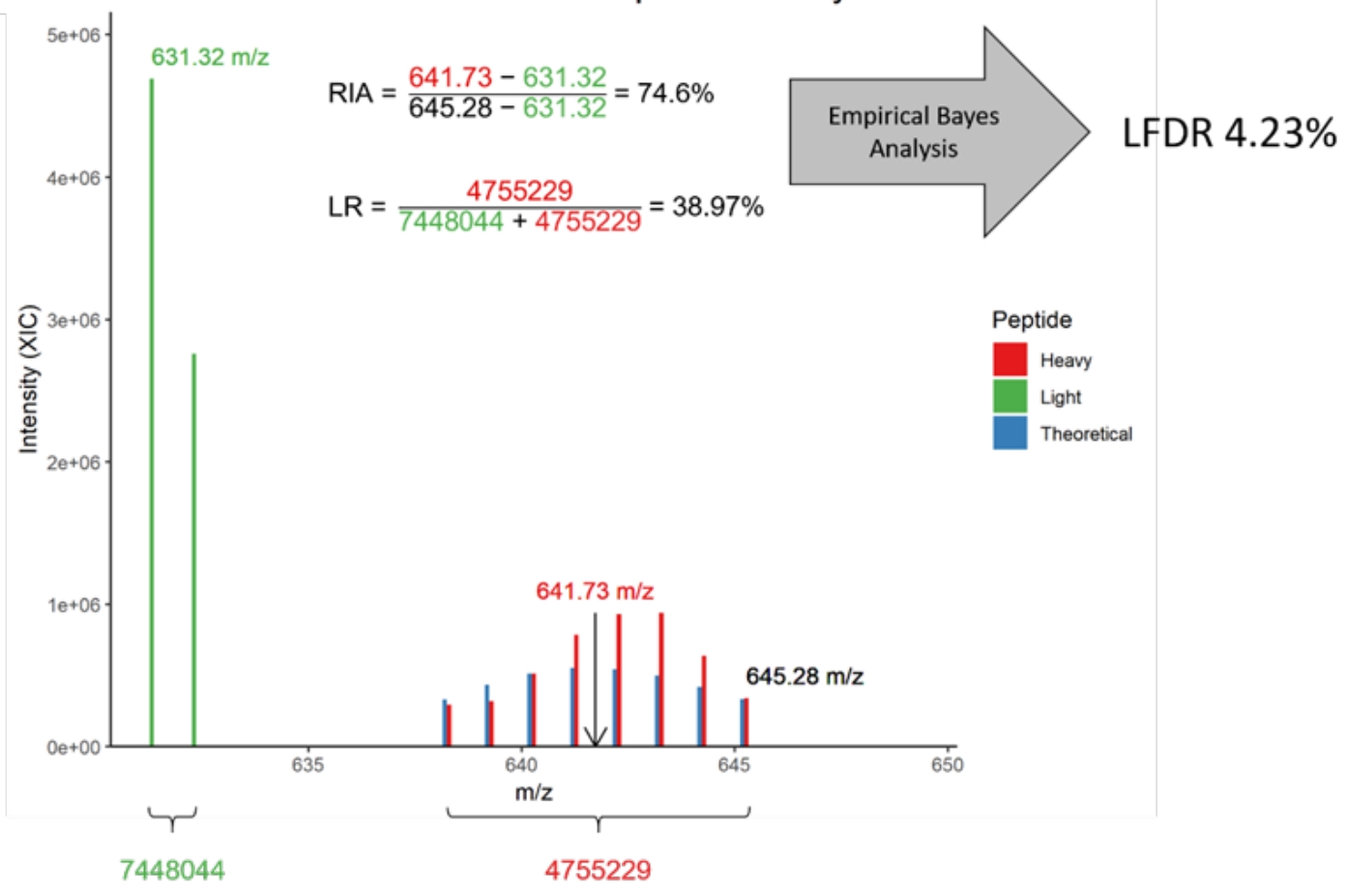


Figure 1.2: Example of how RIA and RITZ were calculated. Once the light peptide feature was extracted from the raw MS file, a theoretical isotopic distribution (blue lines) was determined and compared against the observed peaks to extract the heavy features. Once a match was found, the $\mathrm{m} / \mathrm{z}$ value from the center of the theoretical distribution was chosen for RIA calculation. RITZ is the sum of the peaks of the heavy peptide (red) divided by the total sum of the light (blue) and heavy features. 
substrate would become nearly impossible. This is further complicated by the fact that high concentration of deuterium inhibits enzymatic activity due to kinetic isotope effects. Deuterium also causes significant changes in retention time, making identification of labeled proteins difficult.

The second most abundant element in proteins is carbon. Since carbon is the main component of bacterial biomass, tracking this atom provides an efficient way to assess metabolic activity of microbial communities. Protein-SIP was originally used to identify bacteria that can degrade environmental pollutants, which are mostly carbon-based. For this reason, most SIP experiments are based on ${ }^{13} \mathrm{C}$.

Oxygen is the third most abundant element in amino acids. Surprisingly, there has not been any protein-SIP experiments based on ${ }^{18} \mathrm{O}$ labeled complex substrates or nutrients. The moderate cost and high abundance of oxygen in amino acids would seem to be an attractive option for protein-SIP. In addition, there is negligible effects of ${ }^{18} \mathrm{O}$ in reverse phase liquid chromatography. However, its limited use is due to the exchange of ${ }^{18} \mathrm{O}$ with ${ }^{16} \mathrm{O}$-water by enzymatic reactions, which would reduce its direct incorporation from labeled substrate into biomass(Jehmlich et al., 2016).

Nitrogen ranks fourth in abundance for proteogenic amino acids. This atom has recently gained more use in protein-SIP experiments(Jehmlich et al., 2016). While sensitivity is sacrificed compared to carbon, proteins are enriched in nitrogen compared to overall bacterial biomass. This means that there are fewer spurious peaks on the mass spectrum, in contrast to ${ }^{13} \mathrm{C}$ protein-SIP. It also does not get exchanged with aqueous media like oxygen or hydrogen does. 
Sulphur is the element with the lowest abundance in peptides as it is only present in cysteine and methionine. While tracking the incorporation of Sulphur would provide valuable information, labeling with ${ }^{36} \mathrm{~S}$ isotope is expensive. However, as high-resolution mass spectrometry become more available, tracking with the cheaper ${ }^{34} \mathrm{~S}$ isotope would be possible.(Jehmlich et al., 2016)

\subsection{Other Stable Isotope Probing Approaches}

Aside from protein-SIP, other biomolecules have been used with SIP to study microbial communities(Egert et al., 2007; Herrmann et al., 2017a,b; Kovatcheva-Datchary and Egert, 2009; Young et al., 2015). In RNA- or DNA-based SIP, the incorporation of isotopes into nucleic acids are investigated. By identifying the microbes with heavy DNA or RNA, the cells that are actively replicating can be determined. Since this approach uses metagenomics or metatranscriptomics, there has been more research conducted with DNA and RNA-SIP than with protein SIP. However, the drawback from this approach is that it requires a high degree of labeling as the resolution for observing the change in mass is low. There is also fatty acid-SIP, which uses LC-MS/MS, but phospholipid fatty acids do not contain rich phylogenetic information(Berry and Loy, 2018). Since proteins can be iden-

tified via LC-MS/MS, protein-SIP offers an accurate and sensitive way to determine the incorporation of stable isotopes into proteins. In addition, identified peptide and protein sequences can be used to determine taxa of analyzed organisms. 


\subsection{Applications of Stable Isotope Probing}

Previous research using DNA- and RNA-SIP have been successful in uncovering interactions and roles of gut microbiota(Berry and Loy, 2018). By taking samples at different time points during feeding, we can see which bacteria becomes labeled first to elucidate the effects of cross-feeding. Indeed, this strategy combined with RNA-SIP has been applied in several studies to identify key utilizers of glucose, sialic acid, and resistant starch in gut. Egert et al. (2007) administered ${ }^{13}$ C-labeled glucose to an in vitro human gut model reactor and demonstrated that Streptococcus bovis and Clostridium perfringens were the principal glucose fermenters. Labeled glucose has also been applied to murine gut microbiota where Allobaculum spp. was identified as the active glucose fermenter, with lactate, acetate, and propionate being the principal end-products(Herrmann et al., 2017a). Using

${ }^{13} \mathrm{C}$ labelled potato starch and RNA-SIP, another study analyzed murine stool to show that members of Prevotellaceae and Ruminococcaceae were the main assimilators of resistant starch(Herrmann et al., 2017b). Using the same precursor, one study used an in vitro human model reactor to demonstrate Ruminococcus bromii as the main degrader of starch(Kovatcheva-Datchary and Egert, 2009). RNA-SIP using 13-C sialic acid was also used to identify Prevotella spp. as the key sialic acid utilizer in the piglet cecal microbiota(Young et al., 2015).

While fewer in number than DNA- and RNA-SIP, protein-SIP has also been successful in identifying microbes involved in metabolic processes, as well as contributing insight into the progress behind certain gut dysbiosis. One study intravenously administered ${ }^{13} \mathrm{C}$ labeled leucine to mice and demonstrated that members of Enterobacteriaceae use amino 
acids derived from blood, which may contribute to their growth during small intestinal inflammation associated with parenteral nutrition(Ralls et al., 2016). A similar study, where ${ }^{13} \mathrm{C} /{ }^{15} \mathrm{~N}$-labeled threonine was intravenously administered instead, showed that Akkermansia muciniphila and Bacteroides acidifaciens were the most abundant utilizers of secreted host proteins(Berry et al., 2013). The first study to use total ${ }^{15} \mathrm{~N}$ labeled diet to assess microbial activity in the gut was by Oberbach et al. (2017). In that study, they used protein-SIP to quantify the incorporation of 303 bacterial peptides in colonic mucus of rats fed either a control diet or a high-fat diet with ${ }^{15} \mathrm{~N}$ (Oberbach et al., 2017). They found that Verrucomicrobiaceae and Desulfovibrionaceae were enriched in the active fraction of the community in the high-fat diet. The advantage of using total labeled diet over complex compounds is that analysis is not limited to select microbes as all will intake the labeled diet for growth, which allows to assess the general activity of the microbiome.

\subsection{Challenges in Protein-SIP}

Protein-SIP has not seen as much use in research as DNA-SIP or RNA-SIP due in large part in the limited technologies available for accommodating protein-SIP experiments. Other labeling strategies such as stable isotope labeling of mammals (SILAM; heavy peptides have single labeled lysine when fully digested by trypsin)(Rauniyar et al., 2013), tandem mass tags (TMT)(Thompson et al., 2003), and isobaric tags for absolute and relative quantification (iTRAQ)(Aggarwal et al., 2006), have defined mass shifts, which can be easily implemented as a variable or fixed modification in most search engines. However, in protein-SIP, a single peptide can contain multiple sites that may or may not be labeled. 


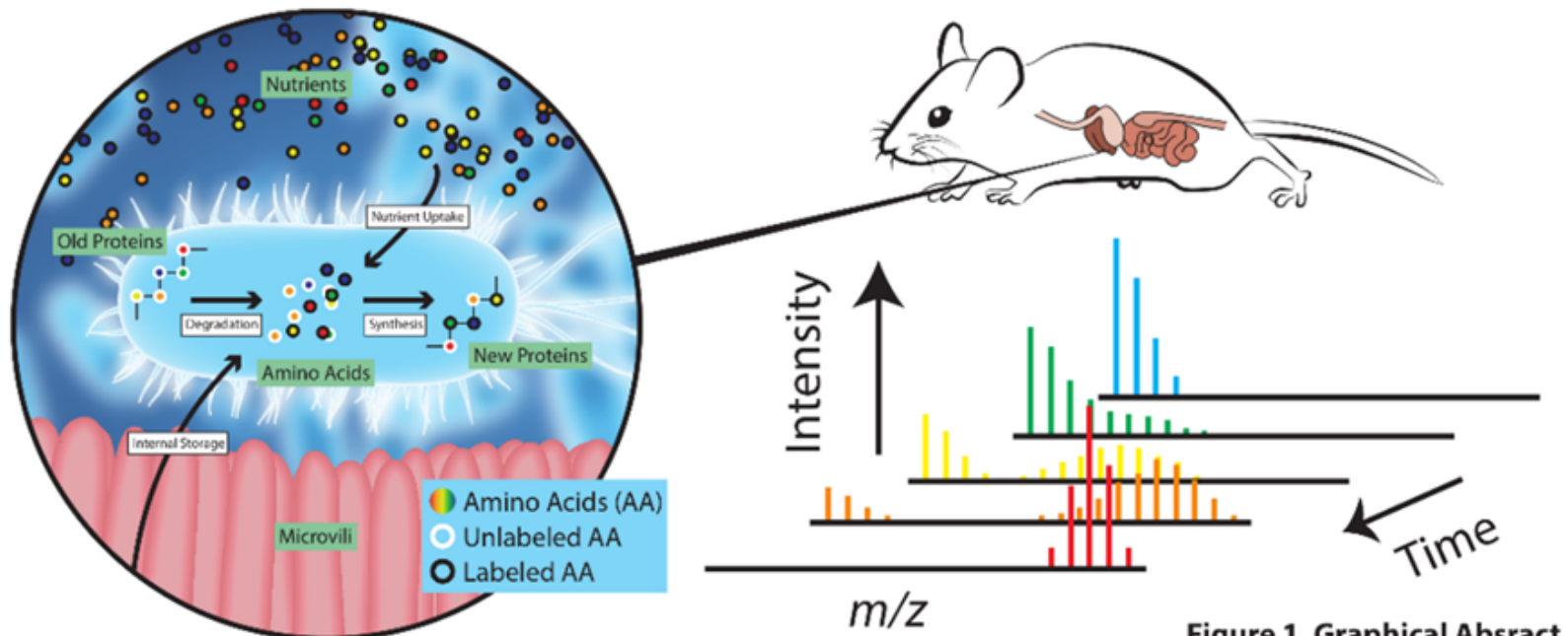

Figure 1. Graphical Absract. 
Figure 1.3: An example of partial labeling in protein SIP experiments. The left figure demonstrates the possible source of amino acid for protein synthesis in gut microbes. The right figure shows an example of a mass spectra containing peaks from a light and heavy peptide over time. The extent of labeling is represented the color change from light blue to red. 
The concept of partial labeling is in part due to processes like host protein degradation, de novo synthesis, and amino acid interconversion,(Fan et al., 2016) which causes the pool of amino acid to sequentially grow towards heavy isotopes as time progresses. An illustration of this concept is shown in figure 1.3. Therefore, since the number of heavy isotopes within a peptide may not be known at one particular time, common database searches would need to accommodate all possible iterations of labeling for each peptide in the database. This can sometimes be feasible when the number of potential labeling sites are low. However, when using substrates like total ${ }^{15} \mathrm{~N}$ labeled diet where all nitrogens in the peptide are potential labeling sites, the search space would be far too big to compute the PSMs in a reasonable amount of time. In addition, due to the large increase in database size, the discrimination power between false and true discoveries will be low, resulting in fewer number of identifications. For this reason, common tools available for labeled peptide identification and quantification, such as MaxQuant(Cox and Mann, 2008), Census(Park et al., 2014), and pQuant(Liu et al., 2014), are not suitable for protein-SIP experiments as they rely on defined mass shifts to generate the list of peptide candidates.

The general strategy to address the issues of partial labeling is to do a database search using only the light peptides and then using their sequence, mass, retention time and charge state to identify their heavy counterparts. This avoids using a very large search space to identify the heavy peptides via MS/MS. Early research using protein-SIP used manual peak extraction and Excel scripts that validated the labeled peptides and isotope patterns(Taubert et al., 2012). However, this process was very laborious and time consuming, often requiring several months of work. Fortunately, several software tools have 
been published that offers automated approaches to labeled protein extraction. The first was by Price et al. (2010) where they used a decomposition algorithm to match theoretical isotope distributions to the observed distributions. They applied this software to measure protein turnover in mouse brain tissue. However, its use is only intended for single cultured organisms and not for heterogenous microbial communities. The second was the SIPROS software by Wang et al. (2013), which is used for protein-SIP experiments using ${ }^{15} \mathrm{~N}$-labeled substrates. The drawback to this software is that, compared to the method by Price et al., it requires large amounts of computational resources. Two additional software were published, SIPPER by Slysz et al. (2014) and MetaProSIP by Sachsenberg et al. (2015) within one year of each other. The SIPPER tool is a software package that requires an external input of molecular formulas and feature positions of ${ }^{13} \mathrm{C}$-labeled peptides. It currently does not support any additional isotopes. MetaProSIP, which is still being updated today, supports ${ }^{15} \mathrm{~N},{ }^{14} \mathrm{C},{ }^{16,18} \mathrm{O}$, and ${ }^{2} \mathrm{H}$ based protein-SIP experiments. This software tool uses the same algorithm to Price et al. (2010), but has features compatible with metaproteomic studies. It is also a part of the OpenMS package suite, allowing for fully automated workflows. The package contains many useful tools for processing mass spectrometry data which enables a high degree of flexibility.

In the study by Oberbach et al. (2017), they used MetaProSIP to extract the heavy peptides from their dataset. However, they were only able to identify 303 heavy peptides from 1,850 light peptides due to light peptides disappearing in later timepoints. In this study, I developed a metaproteomic workflow that addresses this issue by spiking unlabeled proteins from the sample at time zero to the pool of labeled proteins to ensure the contin- 
uous presence of a light peptide. In addition, I minimized the number of false positives by computing a local false discovery rate for each heavy petpide feature extracted.

\subsection{Local False Discovery Rate}

One particular issue with doing biological interpretation from proteomic data is that some peptide identifications can be the result of a false positive, which in the case of partial labeling studies can that the form of heavy features quantified from co-eluting peptides and experimental/bioinformatic noise. Thus, confidence scores are often reported with proteomic data so that confident peptide identifications can be obtained. However, no such value is provided for protein-SIP experiments. Thus, in order to estimate a confidence score for protein-SIP data, we can use the idea that the probability of obtaining high RIAs and LRs at early timepoints in a given time series experiment is lower than the probability of obtaining high RIAs and LRs at later timepoints. In addition, we can also use the idea that the distribution of RIA and LR at time zero will reflect the distribution of false discoveries at later timepoints. It is important to note that some deviations between the distribution of RIA and LR at time zero and the distribution of RIA and LR for false discoveries at later timepoints is expected since that, in later timepoints, some of the heavy peptides might co-elute with the light peptide of another, which will be not be reflected at time zero. 


\subsection{Project Aims}

The aims of this project were to (1) develop a bioinformatic workflow that can extract heavy peptides from partially labeled proteomics data; (2) build a tool for analyzing and acquiring confident protein-SIP data, and (3) apply these tools for the analysis of proteome and microbial dynamics in mouse gut microbiome. Namely, in this study, I investigate the extent to which the metaproteome is labeled with ${ }^{15} \mathrm{~N}$ to find key species responsible in various metabolic functions as well as finding the principal assimilators of dietary nitrogen. Not only was I successful in aims (1) and (2), the workflow and software greatly enhanced the number of peptides identified and quantified with ${ }^{15} \mathrm{~N}$ incorporations from the previous study by Oberbach et al. (2017). This provided deeper insights into the functionality of the gut microbiome, thus fufilling aims (3). 


\section{Chapter 2}

\section{Materials and Methods}

\subsection{Mouse experiment and stool sample collection}

The animal experiments were performed at the Ottawa Hospital Research Institute by Xu Zhang, Janice Mayne, Jasmine I. Moore, and Krystal Walker. The animal use protocol was approved by the Animal Care Committee at the University of Ottawa and conducted in strict accordance with the guidelines on the Care and Use of Experimental Animals of Canadian Council on Animal Care (CCAC). A total of five male C57BL/6J mice (Charles River, Sherbrooke, QC) were housed individually in the same room at $25 \circ \mathrm{C}$ with a strict 12-h light/dark cycle. Food and water were available ad libitum. Mice were acclimatized to the facility for 2 weeks and fed a normal chow diet (containing $18 \%$ fat by energy; Harlan Laboratories, Inc., Madison, WI). The diet was then switched to ${ }^{15}$ N-labelled SILAM Mouse Diet (Product no.: 231304650; Silantes GmbH, Munich, Germany) for 43 days, which is a ${ }^{15} \mathrm{~N}$ labeled hydrolysate from Ralstonia eutropha. This diet is a mixture of 
protein-free mouse chow with biomass of ${ }^{15}$ N-labeled Ralstonia eutropha. Stool samples were collected at days $0,1,2,4,8,12,19,29,34$ and 43 and stored at $-80 \circ \mathrm{C}$ until analysis.

\subsection{Mouse gut microbial protein extraction and trypsin digestion}

Approximately $1 \mathrm{~g}$ of mouse stool sample were suspended in $1.5 \mathrm{~mL}$ of ice-cold phosphatebuffered saline (PBS, pH 7.6) with thorough vortexing (3 to 5 glass beads were added to facilitate suspension of stool pellets). The fecal slurries were centrifuged at $300 \mathrm{~g}, 4 \circ \mathrm{C}$ for 5 min. Supernatants were carefully collected, and the pellets were subjected to the above procedure three times. The supernatant for each sample was combined and then followed by three more centrifugations at $300 \mathrm{~g}$ in $4 \circ \mathrm{C}$ for $5 \mathrm{~min}$ to remove debris. The supernatant was then centrifuged at $14,000 \mathrm{~g}$ in $4 \circ \mathrm{C}$ for $20 \mathrm{~min}$ to pellet bacterial cells. The pellet was washed three times by fully re-suspending them in fresh ice-cold PBS. After the last wash, the supernatant was removed and microbial cells were lysed with $4 \%$ (w/v) sodium dodecyl sulfate (SDS) and $6 \mathrm{M}$ urea in $50 \mathrm{mM}$ Tris-HCl buffer (pH 8.0). A tablet of Roche cOmplete ${ }^{\mathrm{TM}}$ mini protease inhibitor tablet was added per $10 \mathrm{ml}$ lysis buffer. To promote microbial cell lysis, each sample was subjected to three ultrasonications (30 s each with 1 min interval on ice) using Q125 Sonicator (Qsonica, LLC) with an amplitude of $25 \%$. Cell debris were then removed through centrifugation at $16,000 \mathrm{~g}$ in $4 \circ \mathrm{C}$ for 10 min. Proteins were precipitated using a 10-fold volume of acidified acetone/ethanol buffer at $-20 \circ \mathrm{C}$ overnight and washed three times using cold acetone. The precipitated proteins 
were then dissolved in $6 \mathrm{M}$ urea/50 mM ammonium bicarbonate buffer $(\mathrm{pH}$ 8) for protein quantitation using DC ${ }^{\mathrm{TM}}$ protein assay (Bio-Rad Laboratories) and trypsin digestion.

Aliquots from each Day 0 samples were combined to generate the unlabeled sample. An equal amount of this light sample was spiked into each heavy labeled sample, generating a 1:1 ratio of light and heavy proteins for in-solution trypsin digestion. Trypsin digestion was conducted as described previously(Zhang et al., 2018a). Briefly, $50 \mu \mathrm{g}$ of proteins were reduced and alkylated with $10 \mathrm{mM}$ dithiothreitol (DTT) and $20 \mathrm{mM}$ iodoacetamide (IAA), respectively. One microgram of trypsin (Worthington Biochemical Corp., Lakewood, NJ) was added to each sample for trypsin digestion at $37{ }^{\circ} \mathrm{C}$ overnight with agitation. The tryptic digest was desalted with a 10- $\mu$ m C18 column and the tryptic peptides were then eluted with $80 \%(\mathrm{v} / \mathrm{v})$ acetonitrile/0.1\% (v/v) formic acid. The eluted peptides were then evaporated using Savant SpeedVac Concentrator and stored at -20॰C for further analysis.

\subsection{Mass Spectrometry Analysis}

The dried tryptic peptides were dissolved in $0.1 \%(\mathrm{v} / \mathrm{v})$ formic acid and the peptides equivalent to $2-4 \mu \mathrm{g}$ of proteins were loaded for mass spectrometry analysis on a Orbitrap Elite mass spectrometer (ThermoFisher Scientific Inc.). The separation of peptides was performed on an analytical column $(75 \mu \mathrm{m}$ x $15 \mathrm{~cm})$ packed with reverse phase beads $(1.9$ $\mu \mathrm{m}$; 120-A pore size; Dr. Maisch GmbH, Ammerbuch, Germany). A 2-hr gradient was performed from 5 to $35 \%(\mathrm{v} / \mathrm{v})$ acetonitrile containing $0.1 \%(\mathrm{v} / \mathrm{v})$ formic acid at a flow rate of $200 \mathrm{~nL} / \mathrm{min}$. The instrument method consisted of one full MS scan from 300 to $1800 \mathrm{~m} / \mathrm{z}$ using an Orbitrap mass analyzer followed by data-dependent MS/MS scan of the 
20 most intense ions using a LTQ Velos Pro mass analyzer. A dynamic exclusion repeat count of 1 and repeat exclusion duration of $30 \mathrm{~s}$ was applied. All data were recorded with the Xcalibur package and exported as RAW format for further analysis. Light and Heavy Peptide Identification and Quantification

For light peptide identification, I identified peptides and assigned them with taxonomic and functional information using MetaLab(Cheng et al., 2017). The platform uses the MaxQuant(Cox and Mann, 2008) package and a target-decoy approach for peptide identification. The protein database used was derived from the catalog of the mouse gut metagenome, by Xiao et al. (2015). A sample specific database was generated using the MS/MS clustering approach from MetaLab(Cheng et al., 2017). This database was in silico digested using trypsin, with two allowed missed cleavages and peptide length of 7 to 42 amino acids long. Fixed modification included carbodimethylation of cystein and variable modifications included acetylation (Protein N-term) and oxidation (M). The ppm tolerance was set at 10 .

For heavy peptide identification and quantification, I used a modified template pipeline (original template available at https://sourceforge .net/projects/open-ms/files/Papers/ MetaProSIP) on the KNIME platform(Berthold et al., 2009), which uses tools from the open source package suite, OpenMS(Alka et al., 2019). The difference of our pipeline from the original is that no mass calibration was used and retention time (RT) alignment used MapAlignerIdentification instead of MapAlignerPoseClustering since the light protein spike-in can be used as a point of alignment for each run. In addition, parameters in the tool MetaProSIP were changed so that it would search for ${ }^{15} \mathrm{~N}$ labeled peptides 
instead of oxygen or hydrogen. The KNIME file describing the pipeline is available at https://github.com/northomics/MetaProfiler.git.

Since the MaxQuant files generated from MetaLab are not compatible with the input files for the OpenMS tools, I developed an R script that can convert result files from MaxQuant into idXML. Feature selection, i.e. the group of peaks along the $\mathrm{m} / \mathrm{z}$ and RT dimension that belongs to a single peptide, is performed using FeatureFinderMultiplex(Alka et al., 2019), which specializes in finding unlabeled peptide features in labeling experiments. The features were then assigned with a peptide sequence using IDMapper(Alka et al., 2019).

In order to combine all the features from each sample into a single master table, RT alignment was performed using MapAlignerIdentification(Alka et al., 2019). Features were then linked using FeatureLinkerUnlabeledQT(Weisser and Choudhary, 2017). Linked features that contain conflicting peptide sequence information were resolved by keeping the sequence with the best score, which in this case is the peptide with the lowest Posterior Error Probability (PEP)(Cox and Mann, 2008) as reported by MaxQuant. This step was done with IDConflictResolver(Alka et al., 2019). These light features were then used to identify their heavy counterparts using the MetaProSIP tool(Sachsenberg et al., 2015). The correlation threshold was set at 0.2 as it ensured that the heavy peptide feature was selected from each mass spectrum. False discoveries were filtered from data using local false discovery rate. The detailed pipeline is illustrated in Figure 2.1.

Quantification was done through a relative ratio between the light peptide and the estimated intensity of the heavy peptide, which is termed labeling ratio (LR) by MetaProSIP. 
However, since proteins from day 0 was spiked in every sample, the labeling ratio in this experiment instead measures the proportion of proteins that are produced using the heavy nitrogen from hydrolysate relative to day 0 . Therefore, I will refer to this value as RITZ to differentiate it from LR. By taking this measure over time, the rate of newly synthesized proteins that incorporate the heavy nitrogen from hydrolysate can be estimated. RITZ is calculated the same way as labeling ratio 1.1. An example of how RIA and RITZ is measured is illustrated in Figure 1.2B.

\subsection{Extracting the Heavy Peptide Features from Protein- SIP Experiments}

Once the features with their assigned IDs are obtained, each sequence is broken down into their elemental composition. Since this study uses total $15 \mathrm{~N}$ labeled diet, the number of nitrogen atoms defines the maximum number of heavy isotopes that can be artificially introduced (i.e., yield a fully labeled peptide). In addition, the presence of naturally occurring isotopes (NOI) of other elements such as carbon or hydrogen will generate additional peaks. Thus, the maximum number of isotopic peaks that are expected for the fully-labeled peptides would be equal to the number of nitrogen plus the additional peaks from NOIs (which I define as five). With this, to calculate the $\mathrm{m} / \mathrm{z}$ positions of the isotopic peaks, $p_{j}$, one can use equation 2.1(Sachsenberg et al., 2015)

$$
p_{j}=m_{k}+j \frac{D_{e}}{z},
$$


where $m_{f}$ is the monoisotopic $\mathrm{m} / \mathrm{z}$ of the feature $k, j$ is the number of isotopes being considered for the feature, $D_{e}$ is the mass difference between heavy and light isotope of the labeling element $e$ in the peptides, and $z$ is the charge of the feature. For the observed peak to be extracted, the difference in the $\mathrm{m} / \mathrm{z}$ and retention time position between the feature and the observed peak must be within a user defined threshold.

When dealing with complex samples, overlap between coeluting peptides or contaminants with the isotopic peaks are going to be expected. To address this, MetaProSIP calculates the Pearson's correlation between the elution profile of the monoisotopic and the putative isotopic peaks in order to detect signals originating from other analytes. If the observed peaks are coming from the real isotopic mass traces, then the Pearson's correlation coefficient will trend towards one. If the peaks are overlapped with spurious signals are it differs in elution profile, then the coefficient will trend towards zero. MetaProSIP allows the users to specify the threshold for the signals to be retained.

\subsection{Decomposition algorithm}

Once the peaks have been selected, a vector of all isotope intensities, $\mathrm{y}=\left[\mathrm{y}_{1}, \ldots, \mathrm{y}_{\mathrm{n}+\mathrm{a}}\right]$, is obtained, where the size will equal to the maximum number of isotopes that can be artificially introduced, $n$, plus the number of additional peaks corresponding to NOIs, $a$. In order to resolve the peaks belonging to the heavy peptides, Price et al. (2010) and Sachsenberg et al. (2015) both use a decomposition algorithm to approximate the true isotopic distribution. This involves using the elemental composition of the sequence $s$ to obtain a linear combination of theoretical isotope patterns $\phi(s)$. By multiplying the linear 
combination with non-negative weights, $\beta$, you obtain the estimated distribution of the heavy isotopes(Sachsenberg et al., 2015).

$$
y=\phi(s) \beta \equiv\left(\begin{array}{c}
y_{0} \\
\vdots \\
y_{n+a}
\end{array}\right)=\left(\begin{array}{ccc}
\phi_{0,0} & \cdots & \phi_{0, n} \\
\vdots & \ddots & \vdots \\
\phi_{n+a, 0} & \cdots & \phi_{n+a, n}
\end{array}\right)\left(\begin{array}{c}
\beta_{0} \\
\vdots \\
\beta_{n+a}
\end{array}\right)
$$

The columns of $\phi(s)$ represents the number of incoporated isotopes being considered and the row represents the peak position. The variable $n$ represents the maximum number of ${ }^{15} \mathrm{~N}$ that can be incorporated into peptide $s$ and $a$ represents the number additional peaks corresponding to NOIs. Since there are technical variation and noise, determining an exact solution for the equation would not be possible. Thus, in order to solve for the equation, I minimize the squared residual error between the observed, $y$, and the calculated, $\phi(s) \beta$, intensities. The resulting weights and intensities are then used to calculate the relative isotopic abundance (RIA) and relative intensity (RITZ) of the heavy peptide.

\subsection{Filtering Spurious peaks}

While MetaProSIP filters out signals that do not match the elution profile of the monoisotopic peaks, some signals originating from irrelevant analytes may still be retained. In order to remove these signals, the tool uses another correlation score based on the shape 
of the isotopic distribution.

$$
c_{r}\left(y, \phi_{r}(s)\right)=\frac{\sum_{i=0}^{n+a}\left(y_{i}-\bar{y}\right)\left(\phi_{i, r}(s)-\bar{\phi}_{i, r}(s)\right)}{\sqrt{\sum_{i=0}^{n+a}\left(y_{i}-\bar{y}\right)^{2}} \sqrt{\sum_{d=0}^{n+a}\left(\phi_{i, r}(s)-\bar{\phi}_{i, r}(s)\right)^{2}}}
$$

This equation calculates the sample Pearson's correlation coefficient, $c_{r}\left(y, \phi_{r}(s)\right)$, between observed isotope pattern, $y$, and, the theoretical isotope pattern, $\phi_{r}(s)$. The variable $\bar{\phi}_{i, r}$ and $\bar{y}$ corresponds to the mean intensity of the observed and theoretical peak, respectively. If the correlation is not equal or higher than 0.6 , the decomposition weights are discarded.

\subsection{Filtering Heavy Peptide Features using Local False Discovery Rate}

A confidence score can be obtained by using an empirical Bayesian approach to calculate a local false discovery rate (LFDR). LFDR, formulated by Efron et al. (2001), is defined as the probability of the hypothesis being null given the observed data. Let us suppose that, at time $t, p_{0_{t}}$ is the probability of data, $x_{t}$, for false discoveries (the null hypothesis) and $p_{1_{t}}$ is the probability of data for true discoveries (the alternative hypothesis). In addition, let us suppose that $f_{0_{t}}\left(x_{t}\right)$ is the probability density function (pdf) of $x_{t}$ for the null hypothesis and $f_{1_{t}}\left(x_{t}\right)$ is the density of $x_{t}$ for the alternative hypothesis at time $t$. Thus, according

the law of total probability, the mixture density for both hypotheses, $f\left(x_{t}\right)$, is measured using equation 2.4

$$
f\left(x_{t}\right)=p_{0_{t}} f_{0_{t}}\left(x_{t}\right)+p_{1_{t}} f_{1_{t}}\left(x_{t}\right)
$$


By applying Bayes rule to equation 2.4, one can obtain the a posteriori probability that the hypothesis $H$ for the random variable $X_{t}$ with value $x_{t}$ at time $t$ is null $p_{0_{t}}\left(x_{t}\right)$ or non-null $p_{1_{t}}\left(x_{t}\right)$.

$$
\begin{aligned}
p_{0_{t}}\left(x_{t}\right) & =P\left(H=\operatorname{null} \mid X_{t}=x_{t}\right)=p_{0_{t}} \frac{f_{0_{t}}\left(x_{t}\right)}{f\left(x_{t}\right)} \\
p_{1_{t}}\left(x_{t}\right) & =P\left(H=\text { non-null } \mid X_{t}=x_{t}\right) \\
& =p_{1_{t}} \frac{f_{1_{t}}\left(x_{t}\right)}{f\left(x_{t}\right)} \\
& =1-p_{0_{t}} \frac{f_{0_{t}}\left(x_{t}\right)}{f\left(x_{t}\right)}
\end{aligned}
$$

Efron et al. specify that, by definition, the LFDR, $P\left(H=\operatorname{null} \mid X_{t}=x_{t}\right)$, is equal to $p_{0_{t}}\left(x_{t}\right)$. Thus, in order to measure LFDR for protein-SIP data, $p_{0_{t}}, f_{0_{t}}\left(x_{t}\right)$, and $f_{1_{t}}\left(x_{t}\right)$ must be measured, where $x_{t}$ is the observed value of RIA and LR. Since the parameters of the pipeline for quantifying heavy peptide features were the same for each timepoint and that all of the heavy peptide features quantified at time zero are false, the density of RIA and LR for false discoveries can be estimated from time zero using multivariate kernel density estimation to obtain $f_{0_{t}}\left(x_{t}\right)$. Similarly, the probability of false discoveries at a given timepoint can be estimated by fitting both the distribution of false discoveries and the distribution of true discoveries to the observed mixture density using maximum likelihood estimation. Finally, LFDR can be empirically measured by using equation 2.4 and 2.5 .

With all the parameters estimated, a threshold can then be set on the computed LFDR to obtain confident heavy peptide features. It must be noted that the disadvantage to using this approach is that heavy peptide features quantified from unlikely events-for example, 
those that have both higher RIA and LR at early timepoints-will often have high LFDR. Thus, these features will be lost in those timepoints when setting the LFDR threshold low.

In our study, the density of RIA and RITZ for false discoveries was empirically estimated from time zero using multivariate kernel density estimation (KDE), performed by the $\mathrm{R}$ package ks(Chacón and Duong, 2018), since all of the heavy peptide features quantified at

time zero are false. Similarly, the probability of false discoveries at a given timepoint can be estimated by fitting both the distribution of false discoveries, which was measured at time zero, and the distribution of true discoveries, which was assumed to be a bivariate Weibull distribution, to the observed mixture density using maximum likelihood estimation.

With all the parameters set, a threshold can then be set on the computed LFDR to obtain confident heavy peptide features. For this study, features with less than 10\% LFDR were used in downstream analysis.

It must be noted that the disadvantage to using this approach is that heavy peptide features quantified from unlikely events-for example, those that have both higher RIA and RITZ at early timepoints-will often have high LFDR. Thus, these features will be lost in those timepoints when setting the LFDR threshold low.

\subsection{Data Imputation}

In order to predict the RIA or RITZ at missing timepoints, I used equation derived from a three-compartment model and developed by Guan et al. (2012)

$$
\gamma(t)=\left(1+y_{\mu} e^{-\mu t}+y_{v} e-v t+y_{k_{b i}} e^{-k_{b i} t}\right) V_{\max }
$$


where

$$
\begin{aligned}
& \mu=\frac{\left(k_{s t}+k_{0 a}+k_{b t}\right)-\sqrt{\left(k_{s t}+k_{0 a}+k_{b t}\right)^{2}-\left(4 k_{0 a} k_{b t}\right)}}{2}, \\
& v=\frac{\left(k_{s t}+k_{0 a}+k_{b t}\right)+\sqrt{\left(k_{s t}+k_{0 a}+k_{b t}\right)^{2}-\left(4 k_{0 a} k_{b t}\right)}}{2}, \\
& y_{\mu}=\frac{k_{0 a} k_{b i}\left(\mu-k_{b t}\right)}{(\mu-v)\left(\mu-k_{b i}\right) \mu}, \\
& y_{v}=\frac{k_{0 a} k_{b i}\left(v-k_{b t}\right)}{(v-\mu)\left(v-k_{b i}\right) v}, \\
& y_{k_{b i}}=\frac{k_{0 a}\left(k_{b i}-k_{b t}\right)}{\left(u-k_{b i}\right)\left(v-k_{b i}\right)} .
\end{aligned}
$$

They describe that $k_{\mathrm{st}}$ is the total protein synthesis rate constant, $k_{\mathrm{bt}}$ is the total protein degradation rate constant, $k_{0 a}$ is the amino acid out flow rate constant, and $k_{\mathrm{bi}}$, is the individual protein degradation rate constant. Note that $V_{\max }$, the maximum fraction that can be achieved, is added to the equation. For this study, $V_{\max }=100 \%$ in order to convert the fraction into percentages. The four constants that define the three-compartment model are estimated using the $\mathrm{C}++$ code from RcppDE(Eddelbuettel, 2010), which is a package that uses a differential evolutionary (DE) algorithm for minimizing a cost function. The advantage of using DE was that it struck a balance between the robustness of an exhaustive search and the speed of a gradient descent. The least squares approach was used as the cost function. This approach was evaluated using a leave-one-out cross validation (LOOCV) approach. 


\subsection{Data Analysis and Visualization}

Data analysis was performed using our developed tool MetaProfiler. All plots were generated using ggplot2(Wickham, 2016) or ComplexHeatmap(Gu et al., 2016). The phylogenetic tree was generated with GraPhlAn(Asnicar et al., 2015). The optimal number of clusters from the hierarchical clustering approach was determined by the $\mathrm{R}$ package, NbClust(Charrad and Ghazzali, 2014), which reports the best number of clusters by majority vote from 30 clustering indices. To test for over-representation of taxa in each hierarchical cluster, a hypergeometric test was used to assess significance. The total number of non-redundant peptides with more than 4 timepoints were used as background for the hypergeometric test. The p-values were adjusted using the benjamini-hochberg $(\mathrm{BH})$ procedure. The package is available at https://github.com/psmyth94/MetaProfiler.git. The mass spectrometry proteomics data have been deposited to the ProteomeXchange with the dataset identifier PXD017451. 


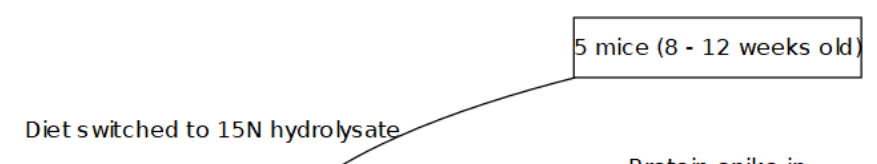

Prote in spike-in
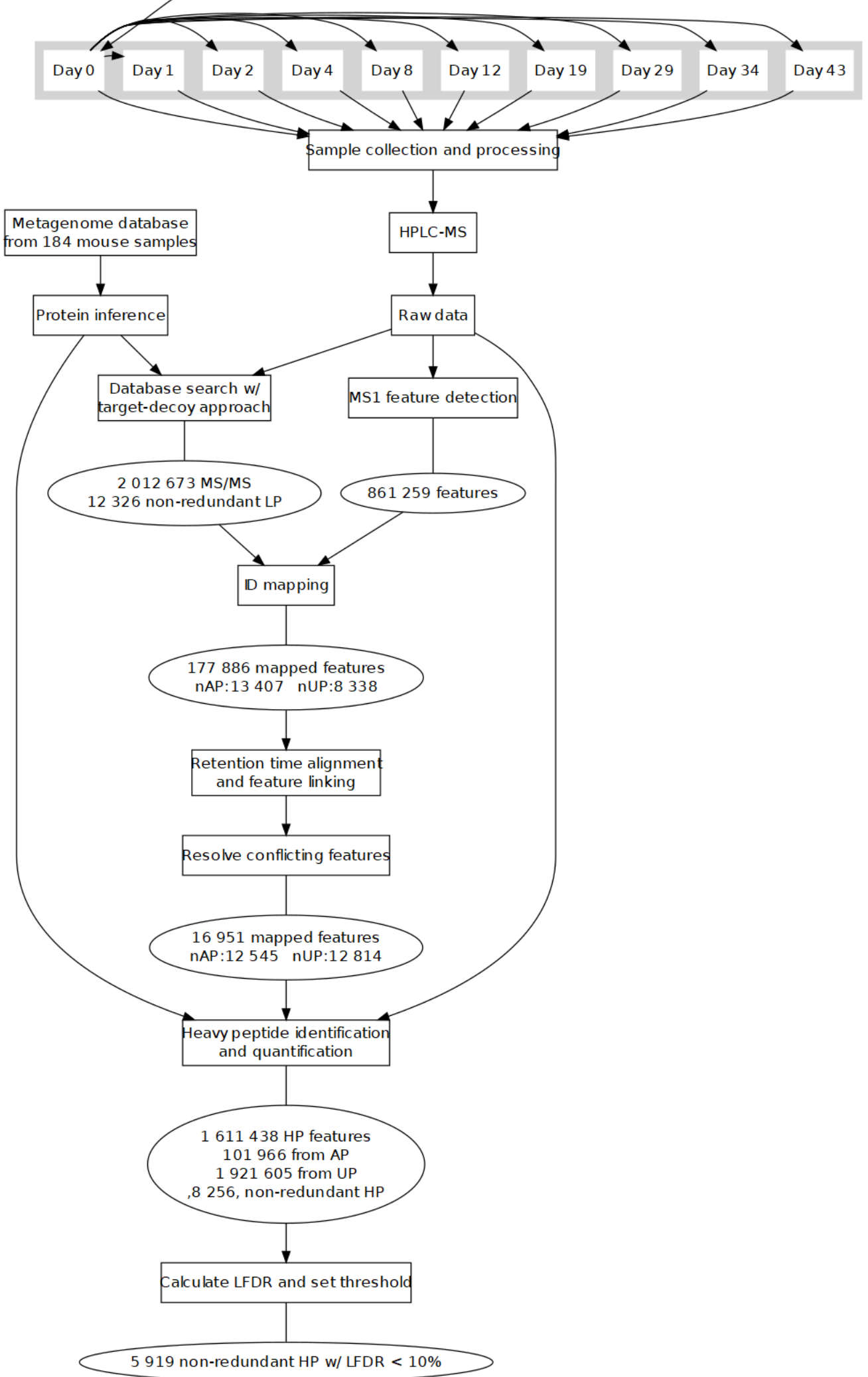
Figure 2.1: Detailed Workflow. Flow chart illustrating the pipeline for identifying and quantifying heavy peptides (HP). LP $=$ Light Peptides; AP = Assigned LP; UP = Unassigned LP; nAP $=$ number of non-redundant LP assigned in at least one sample; $\mathrm{nUP}=$ number of non-redundant LP unassigned in at least one sample. 


\section{Chapter 3}

\section{Results}

Five mice were fed ${ }^{15} \mathrm{~N}$ labeled hydrolysate from Ralstonia eutropha for 43 days, stool samples were collected over 10 time points and their microbiomes were analyzed by metaproteomics (Figure 3.1). From the samples, 10,173 non-redundant heavy peptides corresponding to 2,030,462 features were quantified from the 15,297 non-redundant reference peptide identifications. The pipeline for extracting the heavy peptide features is detailed in the methodology chapter as well as summarized in Figure 3.2A. Peptides were kept if they have at least two RIA values: a value below one over the total number of nitrogen in the peptide,(Kim et al., 2016) $N_{\max ^{-1}}$, which corresponds to the light peptide, and a value above ${ }^{15} N_{\max }{ }^{-1}$, which corresponds to the heavy peptide. After filtering at a threshold level of $10 \%$ local false discovery rate (LFDR), this resulted in 7,108 non-redundant heavy labeled peptides quantified across all samples.

Pairwise correlation on the label free quantification intensity obtained from maxquant for the samples was performed to determine if the runs can be combined in silico. Figure 3.3 

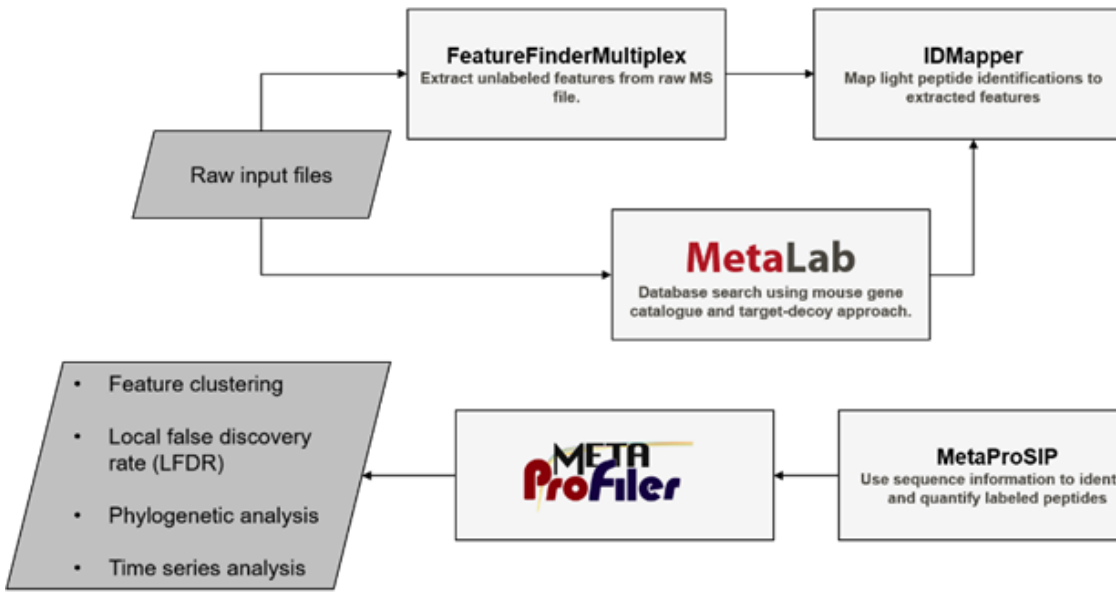

\section{Profiler} MetaProSIP
Use sequence information to identify
and quantify labeled peptides

IDConflictResolver Use highost scoring peptide botwoen identifications. 
Figure 3.1: Bioinformatics Pipeline for Extracting Relative Isotopic Abundance and Relative Intensity from Time Zero. An overview of the workflow for extracting light and heavy peptide features from the raw MS files. 

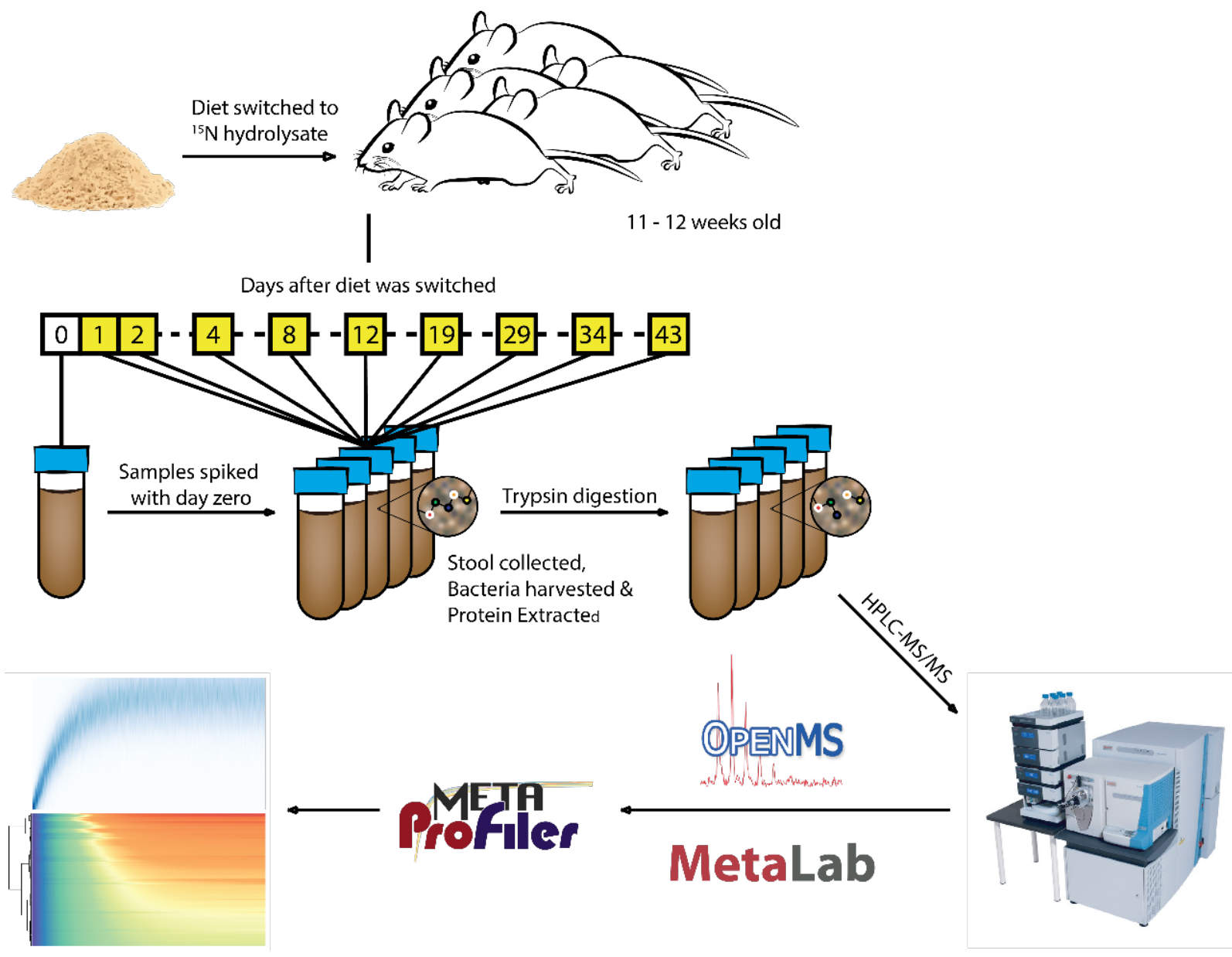
Figure 3.2: Overview of Experimental Workflow. Five 11-12 weeks old male mice were fed with a $15 \mathrm{~N}$ labeled mouse diet, where hydrolysate of the chemolithoautotrophic bacteria, Ralstonia eutropha, was the source of heavy nitrogen. Stool samples were collected at 10 different time points, were spiked with day 0 microbiome, and were processed by mass spectrometry. The mass spectrometry data was analyzed by OpenMS, MetaLab, and MetaProfiler to identify and quantify peptides and proteins and to establish profiles of $15 \mathrm{~N}$ incorporations over time. 


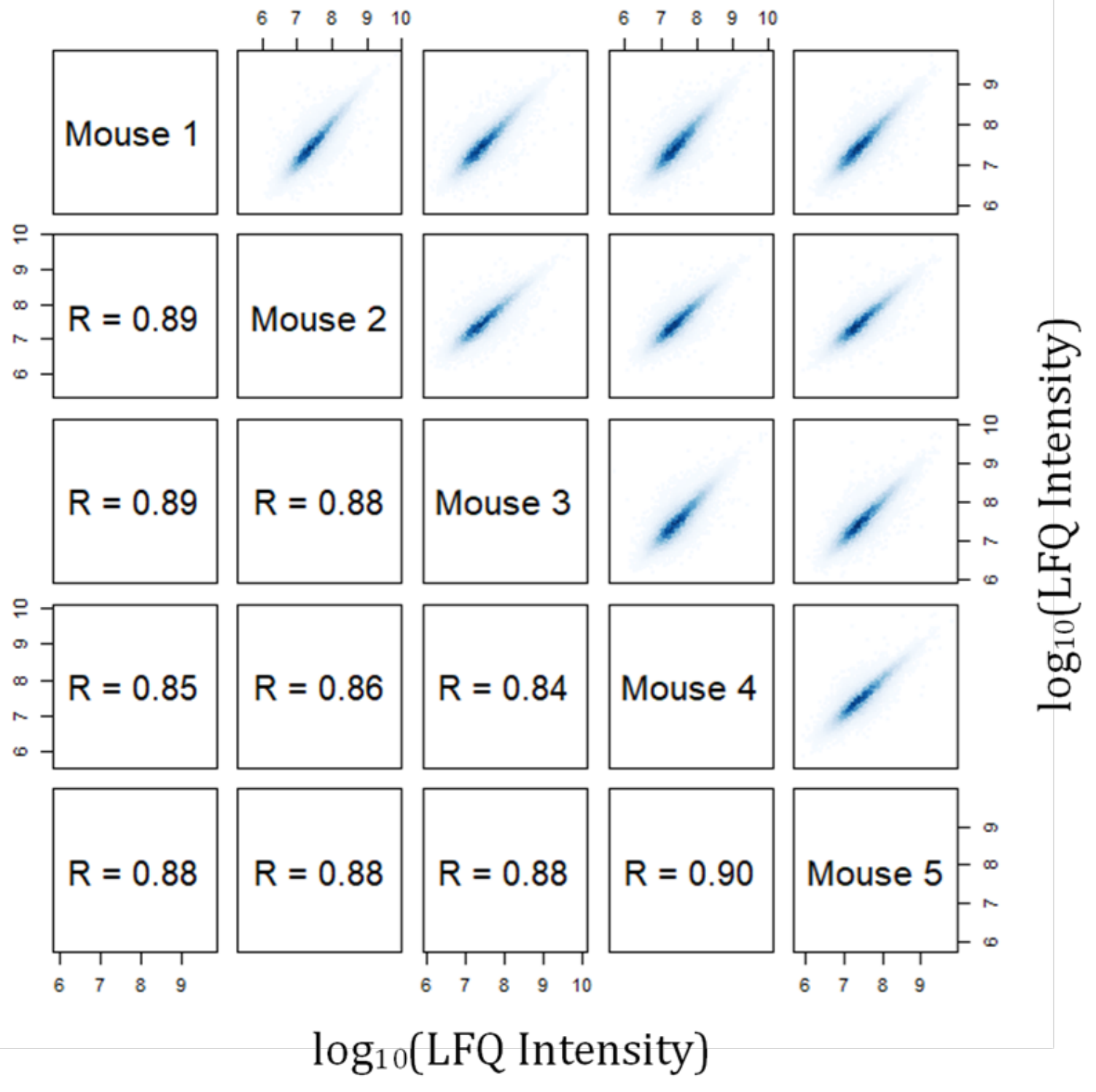


Figure 3.3: The lower panels illustrates the Pearson correlation coefficient between corresponding samples. The upper panels are scatter plots where the axes are the label free quantification (LFQ) intensities obtained from MaxQuant. The color of the points corresponds to their density where blue is the highest value and light blue is the lowest value. 
illustrates the scatter plot and the Pearson correlation coefficient obtained. The smallest value measured is 0.84 denoting that the mouse samples have similar microbiome profiles.

The performances of different workflows were also assessed. When no pooling is done, 2,003 heavy peptides are obtained. Meanwhile, when the timepoints for each sample are pooled together, a slight improvement is seen (3,125 peptides). However, the biggest increase comes from pooling and aligning the samples together, where 10,173 non-redundant heavy peptides are obtained. The need to align the samples together is clearly demonstrated in Figure 3.4, where the retention time can shift from around 5 minutes in different runs. A demonstration of how retention times are aligned is present in figure 3.5.

I also investigated the intensities (Table 3.1), number of identification (Table 3.2), and RIA (Table 3.3) of peptides identified at time zero and those that were not. As seen from Table 3.1, the average $\log 10$ intensity, $\log 10(\mathrm{INT})$, is higher on day 1 for time zero peptides. In Table 3.2, there is an increase in the number of ids from day 1 to day 2 for non-time zero peptides, while you get 2000 less ids for time zero peptides. In addition, $\sim 50 \%$ less non-time zero peptide ids are obtained on day 43 than on day 1 , while for time zero, there is $30 \%$ less. In Table 3.3, the results demonstrates that RIA was significantly lower for non-time-zero peptides from day 4 to day 43 compared to peptides found at time zero. 

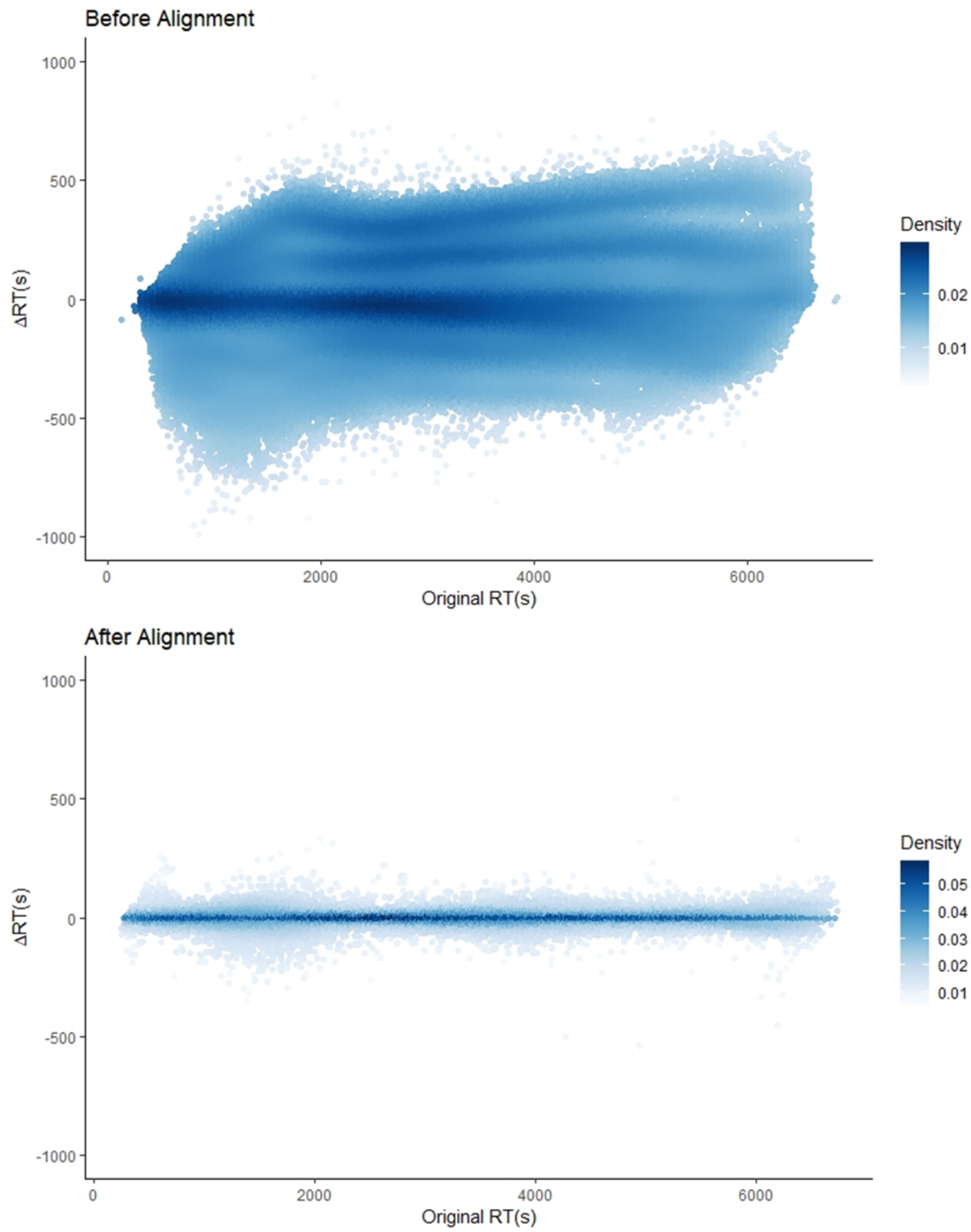
Figure 3.4: The overall retention time drift before and after alignment. The y axis is the difference between the median retention time for a given peptide in all sample and the actual retention time observed in the sample. The $\mathrm{x}$ axis is the actual retention time for a given peptide in a sample. The color of the points corresponds to their density where blue is the highest value and light blue is the lowest value. 

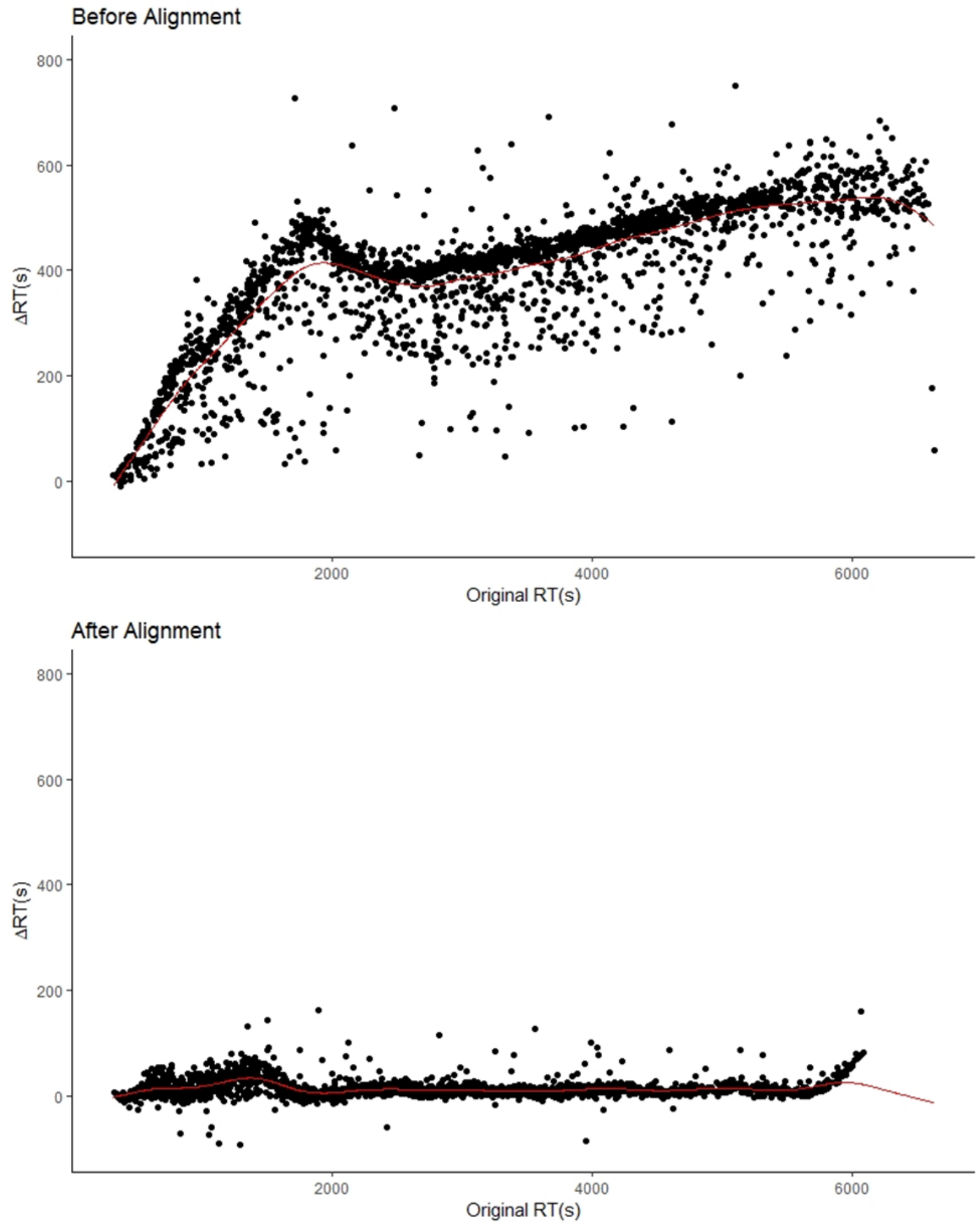
Figure 3.5: The retention time drift before and after alignment for mouse 1 in day 43 . The $\mathrm{y}$ axis is the difference between the median retention time for a given peptide in mouse 1 and the actual retention time observed in the sample. The $\mathrm{x}$ axis is the actual retention time for a given peptide. A line is interpolated using cubic smoothing splines (red line) and then the observed retention time is subtracted by the drift value predicted from the smoothing function. 


\begin{tabular}{lll}
\hline Day & $\begin{array}{l}\text { Found at time zero } \\
(\log 10(\mathrm{INT}))\end{array}$ & $\begin{array}{l}\text { Not found at time zero } \\
(\log 10(\mathrm{INT}))\end{array}$ \\
\hline 0 & 7.2706 & NA \\
1 & 6.9724 & 6.6027 \\
2 & 6.7362 & 6.3368 \\
4 & 6.3861 & 6.3688 \\
8 & 6.5338 & 6.3144 \\
12 & 6.7486 & 6.4897 \\
19 & 6.8771 & 6.5395 \\
29 & 6.7252 & 6.4944 \\
34 & 6.7399 & 6.463 \\
43 & 6.7816 & 6.501 \\
\hline
\end{tabular}


Table 3.1: Average Intensity of Peptides Found at Time Zero and Average Intensity of Peptides Found at Later Time Points. INT represents the average intensity of peptides found at time zero and peptides found at later time points 


\begin{tabular}{lll}
\hline Day & Number of time zero peptides & $\begin{array}{l}\text { Number of non-time zero } \\
\text { peptides }\end{array}$ \\
\hline 0 & 12094 & 0 \\
1 & 7410 & 1066 \\
2 & 5771 & 1160 \\
4 & 5608 & 901 \\
8 & 5581 & 842 \\
12 & 5199 & 657 \\
19 & 4658 & 444 \\
29 & 5223 & 576 \\
34 & 5208 & 610 \\
43 & 5019 & 591 \\
\hline
\end{tabular}


Table 3.2: Number of Peptides Found at Time Zero and Number of Peptides Found at Later Time Points 


\begin{tabular}{llll}
\hline Day & $\begin{array}{l}\text { Average RIA for } \\
\text { time-zero peptides }\end{array}$ & $\begin{array}{l}\text { Average RIA for } \\
\text { non-time-zero } \\
\text { peptides }(\%)\end{array}$ & p-value \\
\hline 1 & 46 & 50.9 & 1 \\
2 & 47.6 & 48.5 & 1 \\
4 & 67.2 & 64.9 & $2 \times 10^{-18}$ \\
8 & 73.8 & 70.9 & $2 \times 10^{-33}$ \\
12 & 77.9 & 75.2 & $7 \times 10^{-32}$ \\
19 & 81 & 76.6 & $7 \times 10^{-41}$ \\
29 & 82.6 & 79 & $2 \times 10^{-43}$ \\
34 & 84 & 80.9 & $2 \times 10^{-42}$ \\
43 & 85.3 & 81.7 & $2 \times 10^{-57}$ \\
\hline
\end{tabular}


Table 3.3: The Average RIA of Peptides Found at Time Zero and Average RIA of Peptides Found at Later Time Points. The p-value is from a Wilcoxon test Correction was performed using $\mathrm{BH}$ procedure. 


\subsection{Taxonomic and functional characterization of the}

\section{heavy labeled peptides in mouse gut microbiome}

To characterize the functions and taxonomic origin of heavy labeled proteins in microbiome samples, I used the confidently quantified heavy peptides at day 29 to 43 . These days were chosen by determining which day the RITZ starts to plateau. Plateau detection was done using a rolling standard deviation, where a window of fixed length moves over the RITZ of each peptide and then computes the standard deviation of the RITZ within the window. The fixed length chosen for the windows was nine days. The average standard deviation across all peptides at each permutation are $9.36 \%$ (day: 1, 2, 4, 8), $7.79 \%$ (day: 4, 8, 12), $6.84 \%$

(day: 12, 19), 5.99\% (day: 29, 34), and 6.23\% (day: 34, 43). Wilcoxon tests showed that the standard deviation in the last window with day 34 and 43 is significantly less than previous windows, except for the second to last one ( $\mathrm{p}$-value $=5 \times 10^{-124}, 2 \times 10^{-49}$, $1 \times 10^{-4}$, and 0.3 , respectively; $\mathrm{BH}$ procedure). Thus, this suggests that the plateau starts at day 29 and the RITZs past this day reflects the maximum proportion of the labeled proteins that the taxon can attain when using hydrolysate as a nitrogen source.

To assess the contribution of the ${ }^{15} \mathrm{~N}$ isotope effect, I compared our dataset against the dataset by Webhofer et al. (2013), where they analysed blood plasma proteins from mice using the same diet as this study. I compared the $\log _{2}\left({ }^{15} \mathrm{~N}\right.$ peptide intensity $/{ }^{14} \mathrm{~N}$ peptide intensity) values of peptides that were in at least three samples and had a p-value from a one-sample t-test less than 0.05 after FDR correction. This is to ensure that the fold 
change is not due to technical variability from the mass spectrometry instrument. The absolute mean $\log _{2}$ value for the Webhofer dataset(Webhofer et al., 2013) is 0.90 at day 56, while this dataset is 2.19 at day 43 , which is significantly higher ( $\mathrm{p}$-value $=1 \times 10^{-122}$ ).

The taxonomic RITZ was first mapped to a phylogenetic tree using GraPhlAn (Figure 3.6). Taxonomic RITZ was taken as the average across all its distinct peptides. Proteins from mice (Chordata) were within the highest peptide RITZ ratios $(\mathrm{RITZ}=0.584 \pm$ 0.020 at $95 \%$ Confidence Interval (CI)). Similarly, Verrucomicrobia, a group of mucin degraders who rely on the host for nutrients, had a similar RITZ level $(0.606 \pm 0.078$ at $95 \% \mathrm{CI}$ ). Three of the four dominant phyla in the gut have mid-range RITZ levels including Proteobacteria $(0.426 \pm 0.079$ at $95 \% \mathrm{CI})$, Bacteroidetes $(0.415 \pm 0.010$ at $95 \%$ $\mathrm{CI})$, and Actinobacteria $(0.304 \pm 0.196$ at $95 \% \mathrm{CI})$. Interestingly, the forth and most dominant phyla, Firmicutes, had significantly lower RITZ (0.306 \pm 0.008 at $95 \%$ CI), than the host cells ( $\mathrm{p}$-value $=2 \times 10^{-104}$; two sample t-test; $\mathrm{BH}$ procedure) and Bacteroidetes (p-value $=3 \times 10^{-58}$; two sample t-test; $\mathrm{BH}$ procedure). While most of the taxa belonging to Firmicutes had low RITZ, the RITZ of the lactic acid bacteria group (e.g. Lactobacillus; $0.548 \pm 0.077$ at $95 \%$ CI) was markedly higher. The mean RITZ of each taxon are available in Table A.1.

To further examine the functional distributions of ${ }^{15} \mathrm{~N}$ labeled proteins, taxa were assigned to categories of COGs (Figure 3.7) using the leading razor proteins associated with their distinct peptides, as reported from MaxQuant. Functions of "carbohydrate transport and metabolism" and "amino acid transport and metabolism", "energy production and conversion", as well as "translation, ribosomal structure, and biogenesis" were among the 


\section{Phyla Legend:}

\section{Chordata Bacteroidetes Proteobacteria Deferribacteres Firmicutes Actinobacteria Verrucomicrobia}

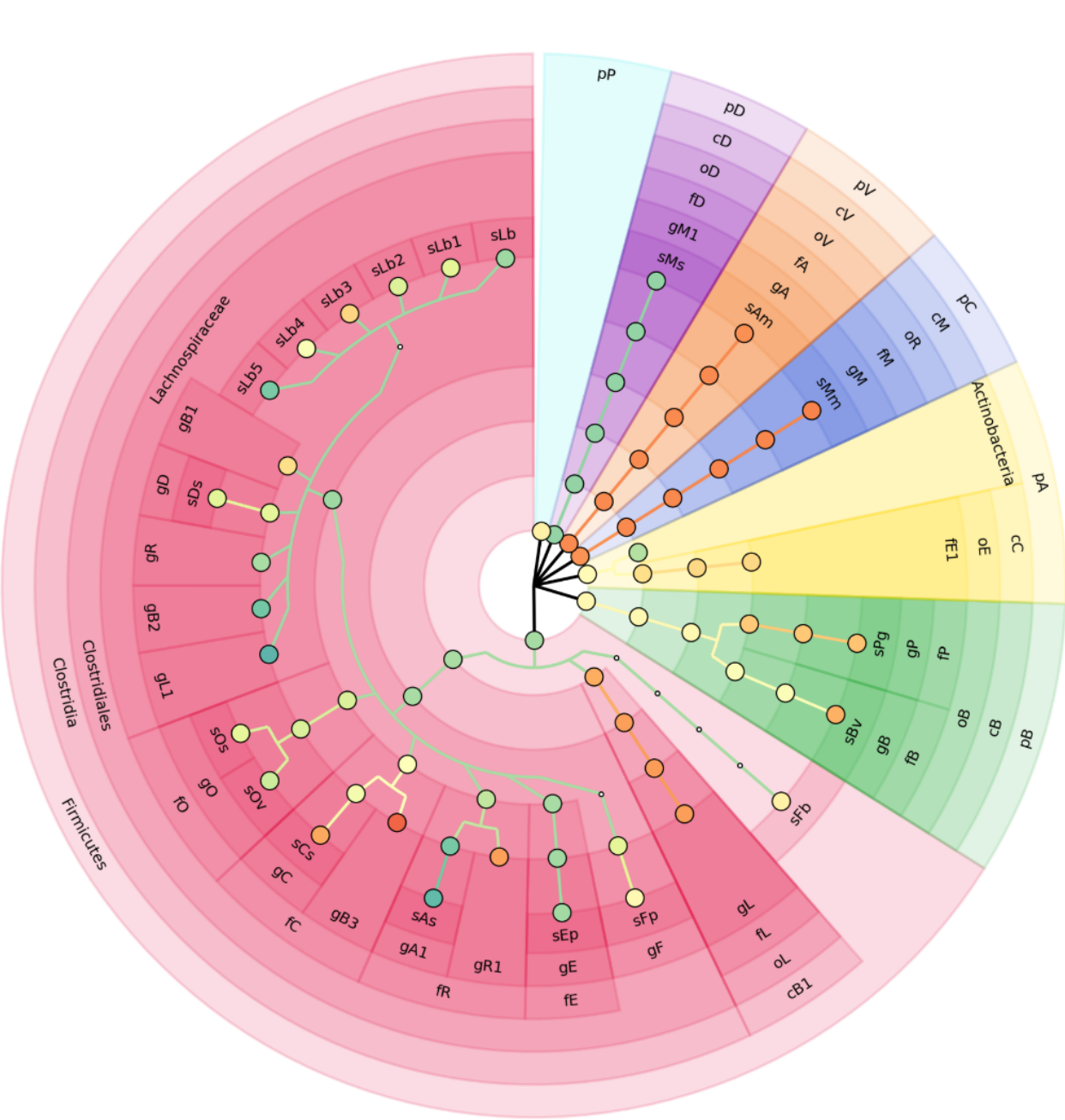

Annotation Legend:

pA: Actinobacteria

pB: Bacteroidetes

pC: Chordata

pD: Deferribacteres

pP: Proteobacteria

$\mathrm{pV}$ : Verrucomicrobia

cB: Bacteroidia

cB1: Bacilli

cC: Coriobacteriia

cD: Deferribacteres

CM: Mammalia

cV: Verrucomicrobiae

oB: Bacteroidales

oD: Deferribacterale

oE: Eggerthellales

oL: Lactobacillales

oR: Rodentia
oV: Verrucomicrobiales fA: Akkermansiaceae fB: Bacteroidaceae fC: Clostridiaceae fD: Deferribacteraceae fE: Eubacteriaceae fE1: Eggerthellaceae fL: Lactobacillaceae fM: Muridae

fO: Oscillospiraceae fP: Porphyromonadacea fR: Ruminococcaceae gA: Akkermansia gA1: Anaerotruncus gB: Bacteroides gB1: Blautia gB2: Butyrivibrio
gB3: Butyricicoccus gC: Clostridium gD: Dorea gE: Eubacterium gF: Flavonifracto gL: Lactobacillus gL1: Lachnoclostridium gM: Mus

gM1: Mucispirillum gO: Oscillibacter gP: Parabacteroides gR: Roseburia gR1: Ruminococcus

sAm: Akkermansia muciniphila sAs: Anaerotruncus sp. G3(2012)

sBv: Bacteroides vulgatus

sCs: Clostridium sp. ASF502
sDs: Dorea sp, 5-2

SEp: Eubacterium plexicaudatum sFb: Firmicutes bacterium ASF500 sFp: Flavonifractor plautii

sLb: Lachnospiraceae bacterium A4 sLb1: Lachnospiraceae bacterium 28-4 sLb2: Lachnospiraceae bacterium COE1 sLb3: Lachnospiraceae bacterium M18-1 sLb4: Lachnospiraceae bacterium A2 sLb5: Lachnospiraceae bacterium 10sMm: Mus musculus sMs: Mucispirillum schaedleri sOs: Oscillibacter sp. 1-3 sOv: Oscillibacter valericigenes sPg: Parabacteroides goldsteinii 
Figure 3.6: Relative Intensity from Time Zero of Proteins in Mouse Gut Microbiome from Day 29 to 43. Phylogenetic tree where size and color of the nodes relates to the RITZ of the taxon; color of the cells corresponds to the phylum lineage; and the transparency denotes the rank. For nodes with annotations, the key follows the convention: [first letter of phylogenetic rank][initials][unique identifier]. Nodes are reported when the number of distinct peptides is greater than 3 and the adjusted p-value from a one-sample t-test is below 0.05 . 
COG Category Legend:

Z: Cytoskeleton

$\mathrm{N}$ : Cell motility

K: Transcription

W: Extracellular structures

Lipid transport and metabolism
. Energy production and conversion

: Amino acid transport and metabolism

F: Nucleotide transport and metabolism

$G$ : Carbohydrate transport and metabolism

M: Cell wall/membrane/envelope biogenesis
J. Translation, ribosomal structure and biogenesis

O: Posttranslational modification, protein turnover, chaperones

Q: Secondary metabolites biosynthesis, transport and catabolism

$\mathrm{U}$ : Intracellular trafficking, secretion, and vesicular transport

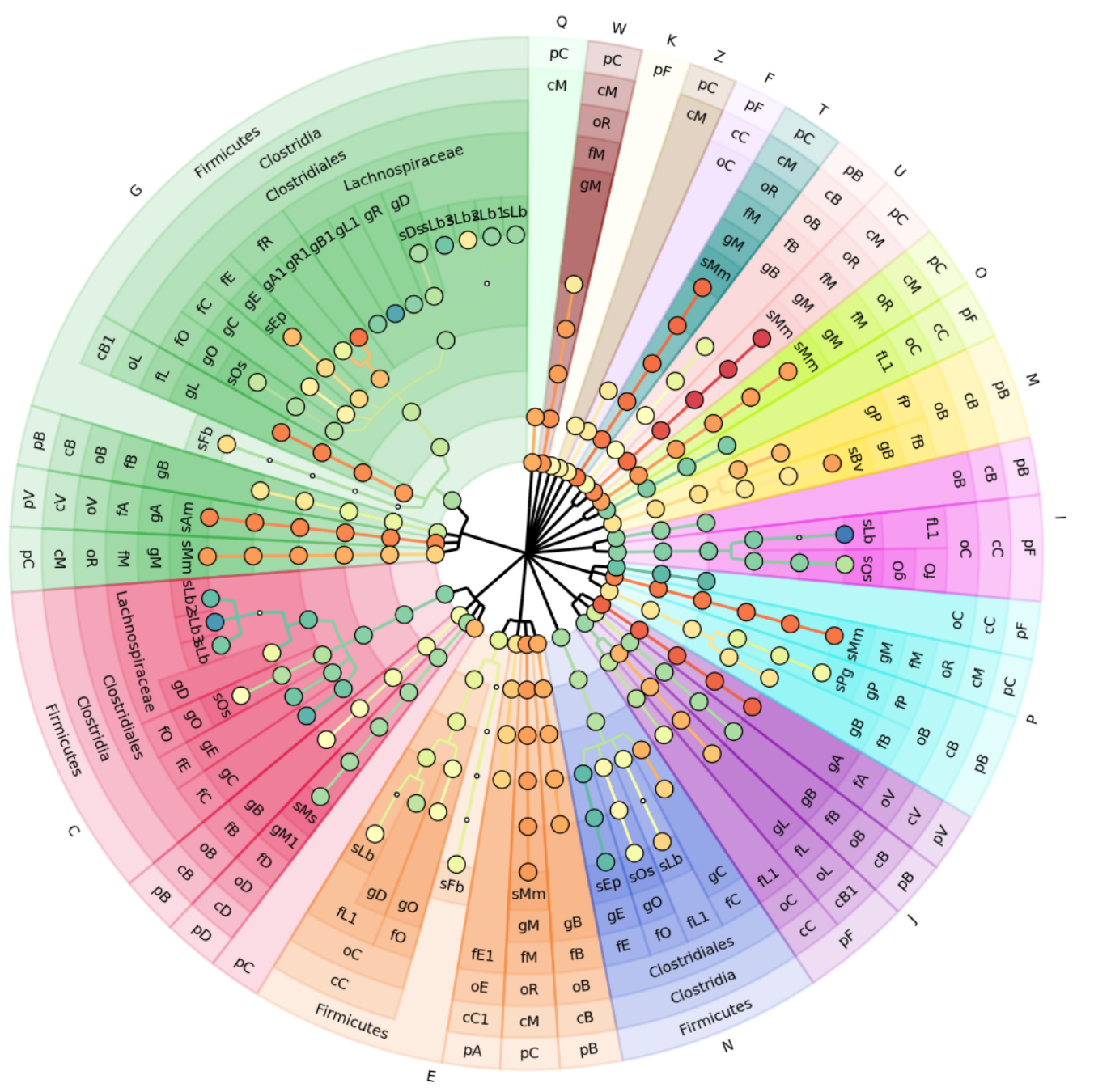

\section{Annotation Legend:}

pA: Actinobacteria pB: Bacteroidetes pC: Chordata pD: Deferribacteres pF: Firmicutes $\mathrm{pV}$ : Verrucomicrobia $\mathrm{CB}$ : Bacteroidia cB1: Bacilli cC: Clostridia cC1: Coriobacteriia cD: Deferribacteres cM: Mammalia cV: Verrucomicrobiae oB: Bacteroidales oC: Clostridiales
oD: Deferribacterales $\mathrm{OE}$ : Eggerthellales oL: Lactobacillales oR: Rodentia oV: Verrucomicrobiales fA: Akkermansiaceae fB: Bacteroidaceae fC: Clostridiaceae fD: Deferribacteraceae fE: Eubacteriaceae fE1: Eggerthellaceae fL: Lactobacillaceae fL1: Lachnospiraceae fM: Muridae

fO: Oscillospiraceae
fP: Porphyromonadaceae fR: Ruminococcaceae gA: Akkermansia gA1: Anaerotruncus gB: Bacteroides gB1: Butyrivibrio gC: Clostridium gD: Dorea gE: Eubacterium gL: Lactobacillus gL1: Lachnoclostridium gM: Mus gM1: Mucispirillum gO: Oscillibacter gP: Parabacteroides
gR: Roseburia gR1: Ruminococcus sAm: Akkermansia muciniphila $\mathrm{sBv}$ : Bacteroides vulgatus sDs: Dorea sp. 5-2

sEp: Eubacterium plexicaudatum sFb: Firmicutes bacterium ASF500 sLb: Lachnospiraceae bacterium A4 sLb1: Lachnospiraceae bacterium COE1 sLb2: Lachnospiraceae bacterium 28-4 sLb3: Lachnospiraceae bacterium 10-1 sMm: Mus musculus sMs: Mucispirillum schaedleri sOs: Oscillibacter sp. 1-3

sPg: Parabacteroides goldsteinii 
Figure 3.7: Functional Distribution of Heavy Labeled Proteins in Mouse Gut Microbiome from day 29 to 43 . The tree describes the functional distribution of each taxon. The size and color of the nodes relates to the RITZ of the taxon; color of the cells corresponds to the functional COG category; and the transparency denotes the rank. Nodes were annotated and reported in a similar fashion to figure 3 . 
categories with the highest number of heavy peptide associated to them. The mean RITZ of each taxon with their associated COG annotations are available in Table A.2.

The host showed high levels of heavy peptides in most categories. In particular, peptides from "intracellular trafficking, secretion, and vesicular transport" (0.612 \pm 0.056 at $95 \%$ CI), which mostly includes calcium-dependent phospholipid-binding proteins, were among

the highest incorporators of ${ }^{15} \mathrm{~N}$ indicative of newly synthesized proteins. In contrast, peptides from "secondary metabolites biosynthesis, transport, and catabolism" (0.506 \pm 0.293 at $95 \% \mathrm{CI}$ ), which mostly includes multidrug resistant proteins, were among the lowest incorporators of ${ }^{15} \mathrm{~N}$. This was reasonable as these proteins are only expressed in response to drugs(Klaassen and Aleksunes, 2010; Mottino et al., 2000).

I also investigated the light peptide intensities of peptides not identified at time zero and noticed that peptides not identified at time zero are significantly lower in intensity than the ones that were. As seen from Table 3.1, the average log10 intensity, $\log 10(\mathrm{INT})$, is higher on day 1 for time zero peptides.

\subsection{Dynamics of ${ }^{15} \mathrm{~N}$ Incorporation into Peptides of the Mouse Gut Microbiota}

I next explored the ${ }^{15} \mathrm{~N}$ incorporation profiles over time. In particular, I focused on the subset of 2,665 peptides that had sufficient data points to perform time-series modeling (observed in at least 5 time points and in at least 3 samples). I assessed whether the datasets could be modeled using a the three-exponential equation derived by Guan et al. 
A

Relative Isotopic Abundance $\begin{array}{llllll}0 & 20 & 40 & 60 & 80 & 100\end{array}$

before

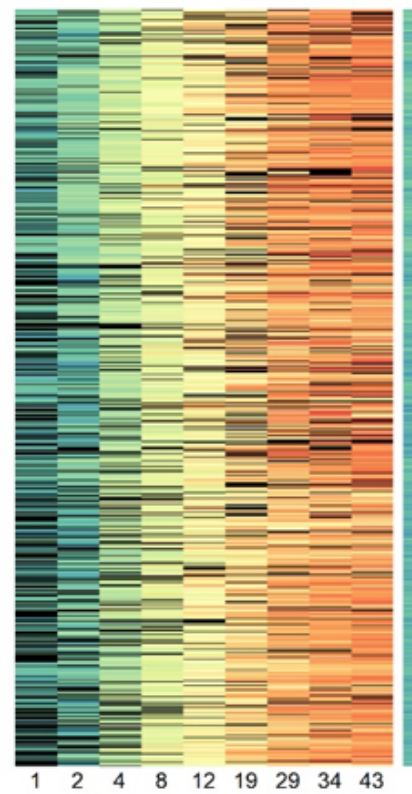

after

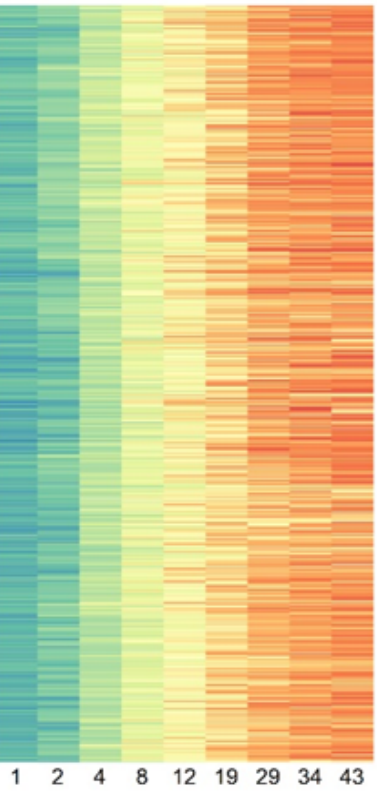

B
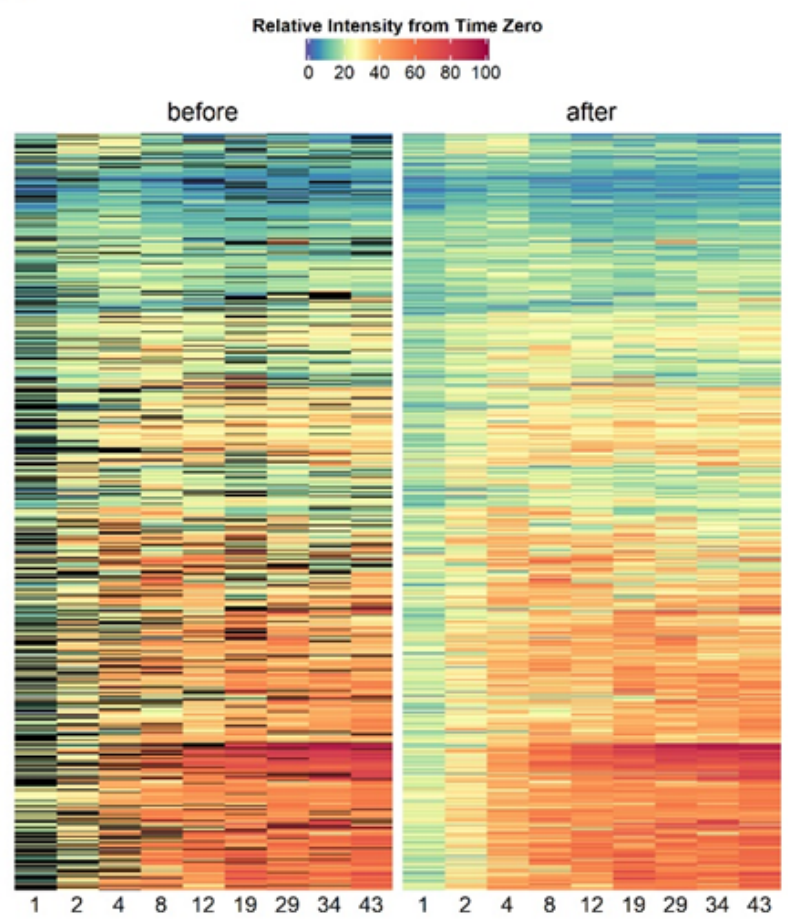
Figure 3.8: Heatmap before and after imputation. A) A heatmap denoting the distribution of relative isotopic abundance (RIA) before and after imputing using the three exponential equation from Guan et al. (2012). The columns are the timepoints and the rows are the averaged RIA of the non-redundant peptides. The color of each cell corresponds to their RIA value, as illustrated by the color key above. B) Similar to A), but with relative intensity (RITZ) instead. 
(2012) for computing protein turnover. This regression model predicted missing values with an average root mean square error (RMSE) of $7.27 \%$ for RITZ and $5.99 \%$ for RIA. The results before and after imputation are available in Figure 3.8.

The overall nitrogen flux was then evaluated in mice gut microbiome (Figure 3.9A). Nitrogen incorporation appeared to be rapid in the first few days but then slowed down (Figure 3.10B). Additionally, the vast majority of peptides did not reach an RIA of 100\%, where the interquartile range was between $88.9 \%$ and $91.8 \%$ at day 43 . To further examine whether there were differences in nitrogen incorporation rate of different peptides, I performed hierarchical clustering using the first four time points (day 1, 2, 4, and 8), where higher RIA variations were observed. Three distinct clusters (RIAC1, RIAC2, and RIAC3) were identified with peptides in cluster RIAC3 showing the fastest rate of isotope incorporation. Interestingly, a noticeable lag in incorporation was present in cluster RIAC1 from day 1 to day 2, and more subtlety in RIAC2. Hypergeometric tests (Table A.3) showed the species that are significantly over-represented in cluster RIAC1 are Bacteroides vulgatus $\left(\mathrm{p}=2 \times 10^{-3} ; \mathrm{BH}\right.$ procedure), host cells (Mus musculus; $\mathrm{p}=3 \times 10^{-4}$; $\mathrm{BH}$ procedure), and Parabacteroides goldsteinii ( $\mathrm{p}=8 \times 10^{-4}$; $\mathrm{BH}$ procedure). To investigate this further, a pairwise t-test between taxa using their distinct heavy peptide RIA accompanied by $\mathrm{BH}$ procedure was performed to identify which species had significantly less nitrogen incorporation at day 2 . From the tests, mouse cells had significantly less incorporation in day 2 than Dorea sp. 5-2 (p-value $=2 \times 10^{-3}$ ), and Lachnospiraceae bacterium $A_{4}\left(\mathrm{p}\right.$-value $\left.=5 \times 10^{-4}\right)$, and Parabacteroides goldsteinii had significantly less incorporation than Dorea sp. 5-2 $\left(\mathrm{p}\right.$-value $\left.=3 \times 10^{-4}\right)$, Eubacterium plexicaudatum $\left(\mathrm{p}\right.$-value $\left.=1 \times 10^{-2}\right)$, 
A

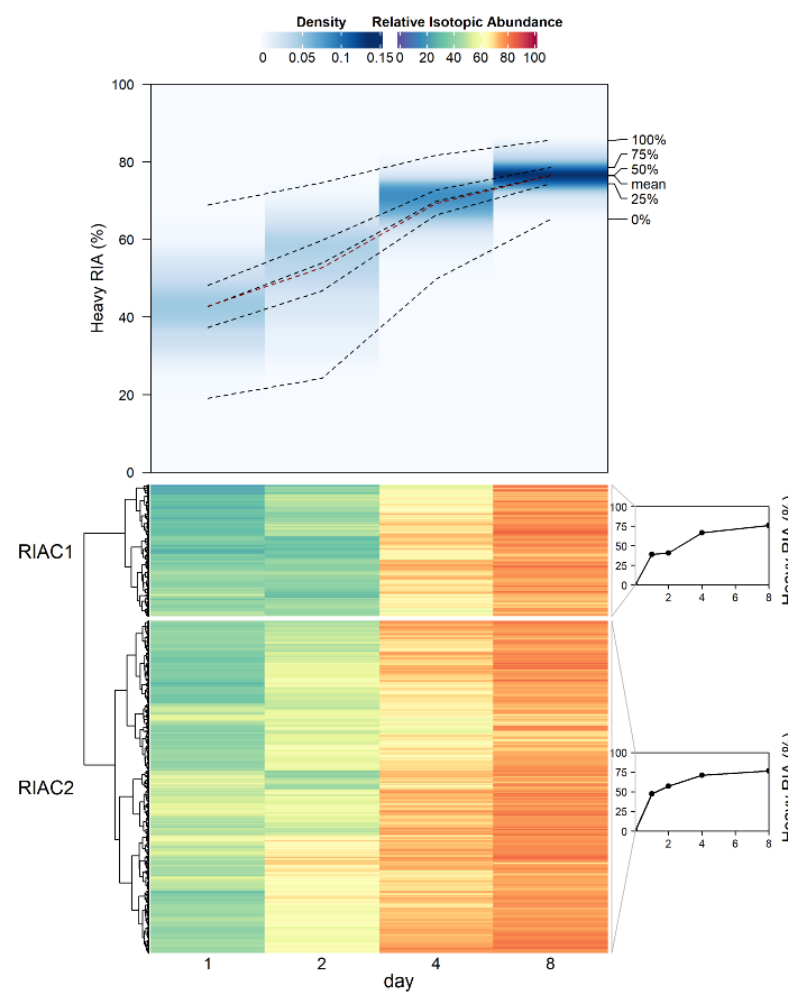

B

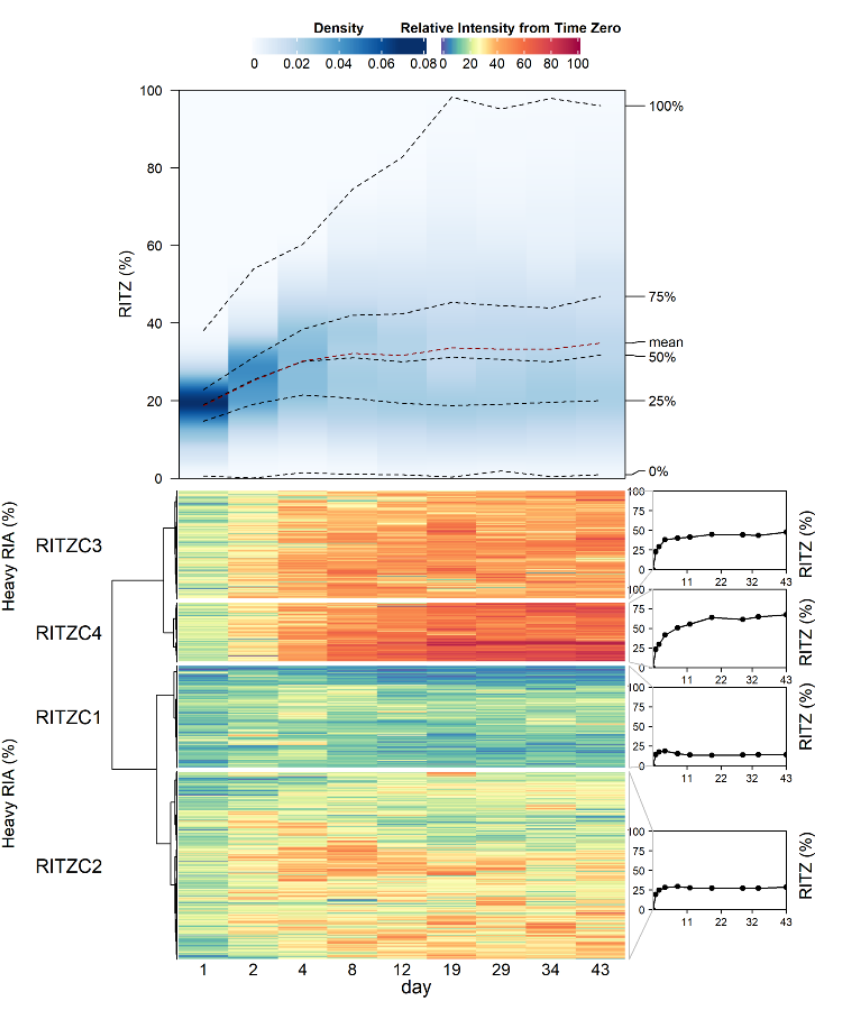


Figure 3.9: Isotope Incorporation Profile. A) The top graph shows the distribution of RIA at each time point. Dark blue denotes a high density and light blue denotes a low density. At the bottom is a heatmap where the rows are peptides and the columns are the days at which the RIA was recorded. B) Similar to A except that it denotes relative intensity over time. Both heatmaps were clustered using Ward's minimum variance, as implemented in,74 using the Euclidean distance. 
A

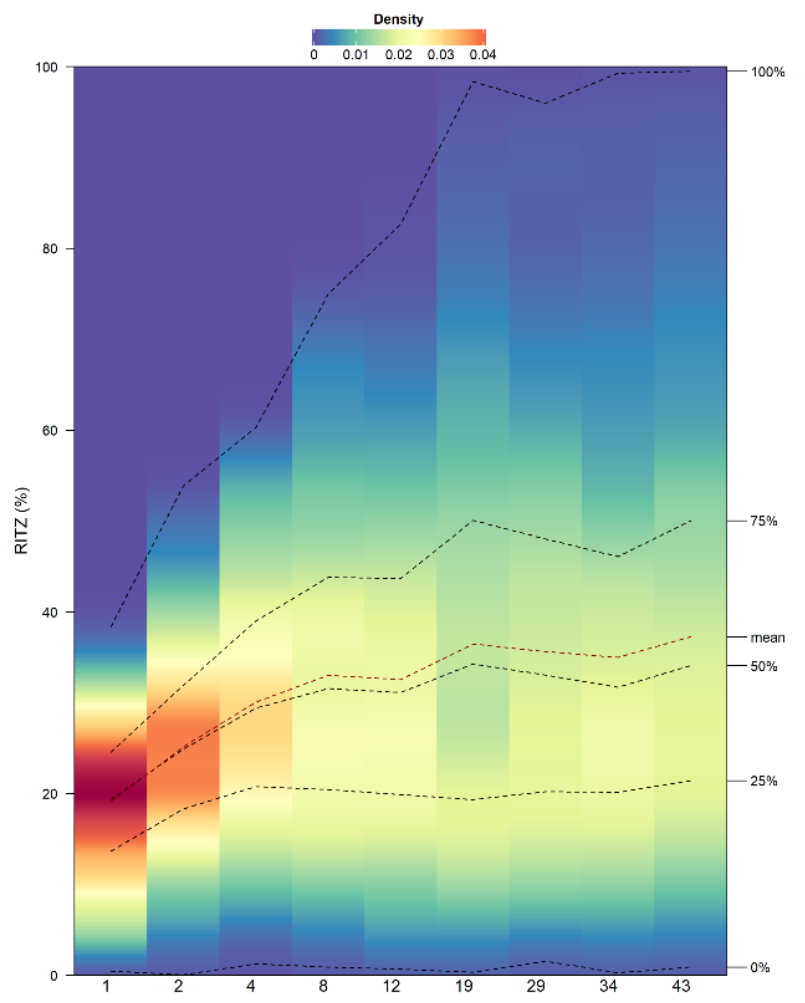

B

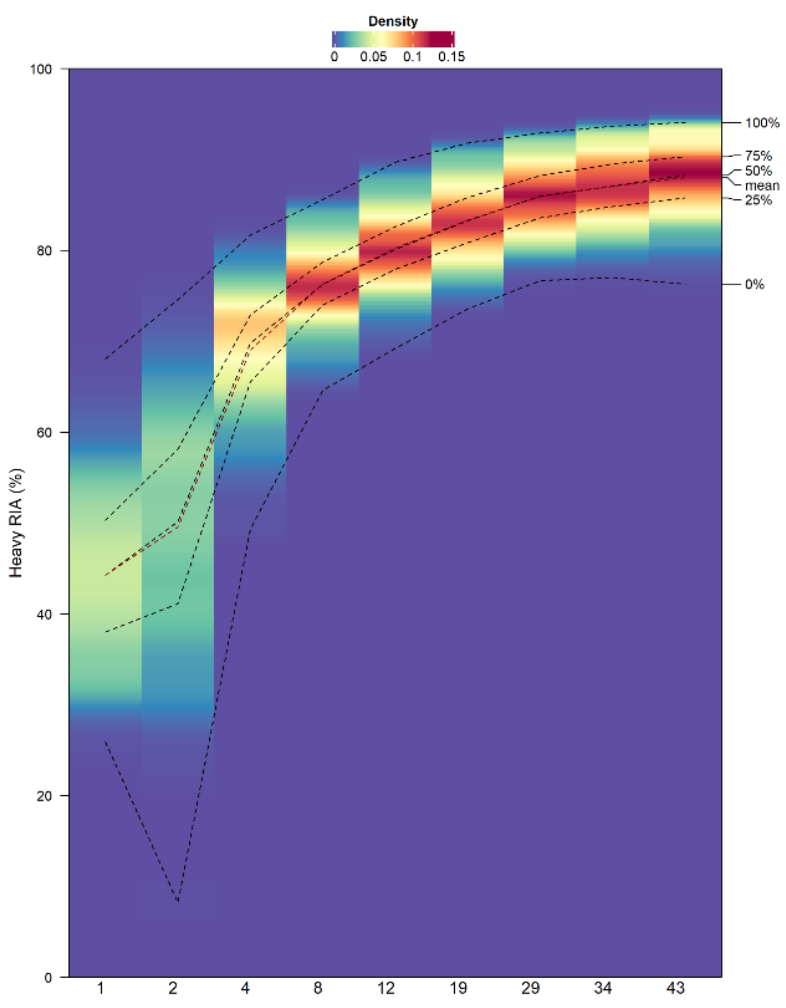


Figure 3.10: RIA and RITZ Distribution of Heavy Peptides Below 10\% LFDR Over Time. The density distribution of A) relative intensity (RITZ) and B) relative isotopic distribution (RIA) at day $1,2,4,8,12,19,29,34$, and 43 . The black line represents the quantile of RIA and RITZ at $0 \%, 25 \%, 50 \%, 75 \%$, and $100 \%$ and the red line represents the mean RIA and RITZ over time. 
Lachnospiraceae bacterium 10-1 $\left(\mathrm{p}-\mathrm{value}=9 \times 10^{-3}\right)$, Lachnospiraceae bacterium 28-4 (pvalue $\left.=3 \times 10^{-2}\right)$, Lachnospiraceae bacterium A4 $\left(\mathrm{p}\right.$-value $\left.=2 \times 10^{-5}\right)$, and Oscillibacter $s p$. 1-3 $\left(\mathrm{p}\right.$-value $\left.=2 \times 10^{-3}\right)$.

In order to group the different rates of newly synthesized proteins, hierarchical clustering of the RITZ over time was performed (Figure 3.9B). A total of four clusters were identified (LRC1, LRC2, LRC3, and LRC4). Peptides from cluster LRC1 showed the lowest RITZ (day $43=0.137 \pm 0.005$ at $95 \% \mathrm{CI}$ ), which became constant after day 4 . Hypergeometric tests (Table A.4) showed that Firmicutes is significantly over-represented in this cluster ( $\mathrm{p}$-value $=4 \times 10^{-5}$; $\mathrm{BH}$ procedure). Cluster LRC2, LRC3, and LRC4 also remained stable until day 43 after the $4^{\text {th }}$ day of ${ }^{15} \mathrm{~N}$ diet feeding, indicating an equilibrium state was reached by these peptides. Peptides from cluster LRC4 reached the highest RITZ ratio at day $43(0.693 \pm 0.015$ at $95 \%$ CI $)$. Hypergeometric tests showed that the taxa over-represented in cluster LRC4 are mouse cells ( $\mathrm{p}$-value $=6 \times 10^{-49} ; \mathrm{BH}$ procedure) and Parabacteroides goldsteinii ( $\mathrm{p}$-value $=2 \times 10^{-3} ; \mathrm{BH}$ procedure). This is consistent with Figure 3.6, where the majority of the mouse cells had high RITZ. Altogether, these findings suggest that the rates of newly synthesized proteins varied among different taxa.

\section{$3.3{ }^{15} \mathrm{~N}$ incorporation rate differed according to mi- crobial phylogeny}

To further investigate whether the incorporation rate of nitrogen differed between microorganisms in mouse gut microbiome, I mapped all the above peptides to taxa and identified 

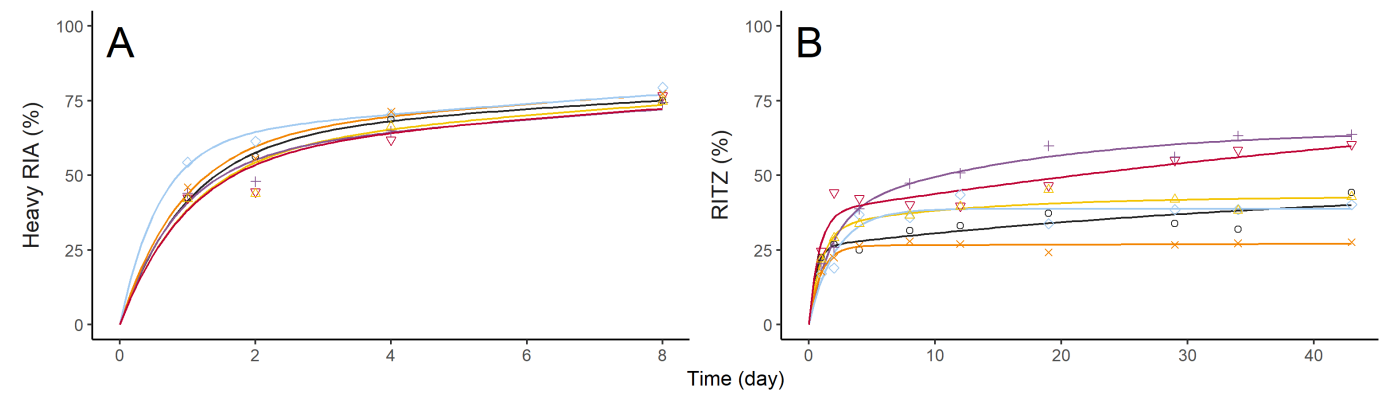

Phylum

- Actinobacteria

$\triangle$ Bacteroidetes

+ Chordata

$*$ Firmicutes

- Proteobacteria

$\rightarrow$ Verrucomicrobia
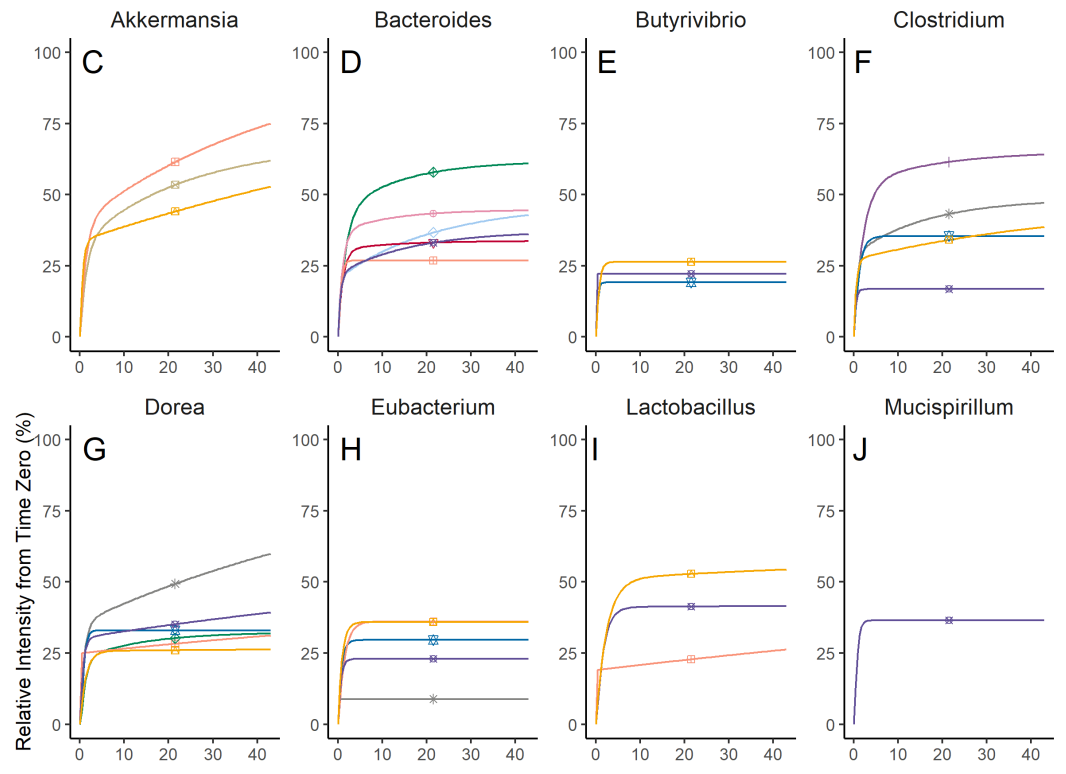

COG category

- Defense mechanisms

- Extracellular structures

- Nucleotide transport and metabolism

* Signal transduction mechanisms

- Cell wall/membrane/envelope biogenesis

* Intracellular trafficking, secretion, and vesicular transport

- Lipid transport and metabolism

* Cell motility

$\leftarrow$ Amino acid transport and metabolism

- - Inorganic ion transport and metabolism

\# Posttranslational modification, protein turnover, chaperones

- Translation, ribosomal structure and biogenesis

Oscillibacter

Parabacteroides

\# Energy production and conversion
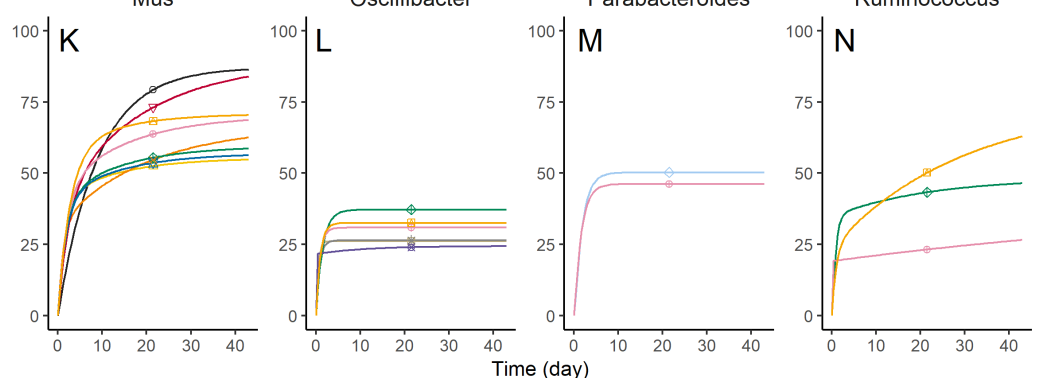

-4- Carbohydrate transport and metabolism 
Figure 3.11: Taxon-Specific RIA and RITZ Profiles Over Time. (A) phylum level RIA and (B) RITZ profiles over time; (C-N) Average COG RITZ profiles over time of the 12 most abundant genera. 
3 superkingdom, 2 kingdom, 9 phylum, 12 class, 12 order, 15 family, 17 genus, and 21 species that have more than 3 distinctive peptides. RIA and RITZ of each taxon at each time point were then calculated using the average of all distinctive peptides (see Appendix A.1.1 for average RIA and RITZ over time for all taxa). At the phylum level, the RIA profiles over time were similar among all the identified seven phyla (Figure 3.11A), with the exception of host cells (Chordata) and Bacteroidetes between day 1 and 2. This further supports the hypergeometric tests performed in the clusters presented in Figure 3.9A. Obvious different RITZ profiles were also observed. The synthesis rate for Firmicutes clearly exhibited a similar pattern found in cluster LRC2 in Figure 3.9B, and reached a lower plateau than other abundant phyla, such as Bacteroidetes, Proteobacteria and the mice host. In agreement with the results obtained above, the mice proteins showed the highest RITZ after 4 days of ${ }^{15} \mathrm{~N}$ diet. These findings suggest that the incorporation of ${ }^{15} \mathrm{~N}$ to the host proteins is faster than the microbiome. Figure 3.11C-N shows the average RITZ overtime across all their COG categories for the most abundant genera.

\subsection{Proteome Dynamics of Arabidopsis thaliana Seedling}

\section{Roots}

MetaProfiler can also be applied to datasets other than microbiome data and without protein spike-ins. Here, the dataset from Gajer et al. (2012) was applied to the same workflow and data analysis. The dataset includes soluble, microsomal, and organellar fractions obtained from Arabidopsis thaliana roots, where the samples were collected after 
$0,4,8,16,24,32,40$, and 48 hours of feeding with ${ }^{15} \mathrm{~N}$ labeled diet. It is important to note that two files are missing in the Pride repository (hour 16 for the soluble fraction and hour 48 for the microsomal fraction). The workflow generated 4,629 non-redundant heavy peptides from 5,086 peptides identified at time zero. In the original paper, they reported the incorporations 1845,1998 , and 6225 peptides in all the time points from the enriched soluble, organellar and microsomal fractions of Arabidopsis seedling roots, respectively. However, after removing peptides not present at time zero, not present in at least 3 time points, and not belonging to a protein with at least 2 unique peptides associated to them, they were left with 345, 321, and 798 peptides, respectively. By applying the same filtering strategy, as well as applying a correlation threshold of 0.6 and LFDR filtering at 10\%, I obtained 662, 660, and 1,433 peptides.

The median LFDR for each time point collected were $99.5 \%, 72.3 \%, 10.5 \%, 2.9 \%, 1.3 \%$, $1.6 \%$, and $1.0 \%$ and the fitted joint distributions of RIA and RITZ are shown in Figure 3.12 for the soluble fraction, Figure 3.13 for the microsomal fraction, and Figure 3.14 for the organellar fraction. As seen from the figures, false discoveries were high in the first two timepoints, causing all the data from hour 4 to be lost and for most to be lost in hour 8. In the paper by Gajer et al. (2012), they mention that some peptides varied in LR in at hour 4 , however they mention that they are no obvious outliers in their datasets. This emphasizes the importance of doing confidence assessments to ensure the quality of the biological interpretations of the results.

In order to group the different rates of protein turnover, hierarchical clustering of the LR over time was performed (Figure 3.15). A total of three clusters were identified (LRC1, 
A
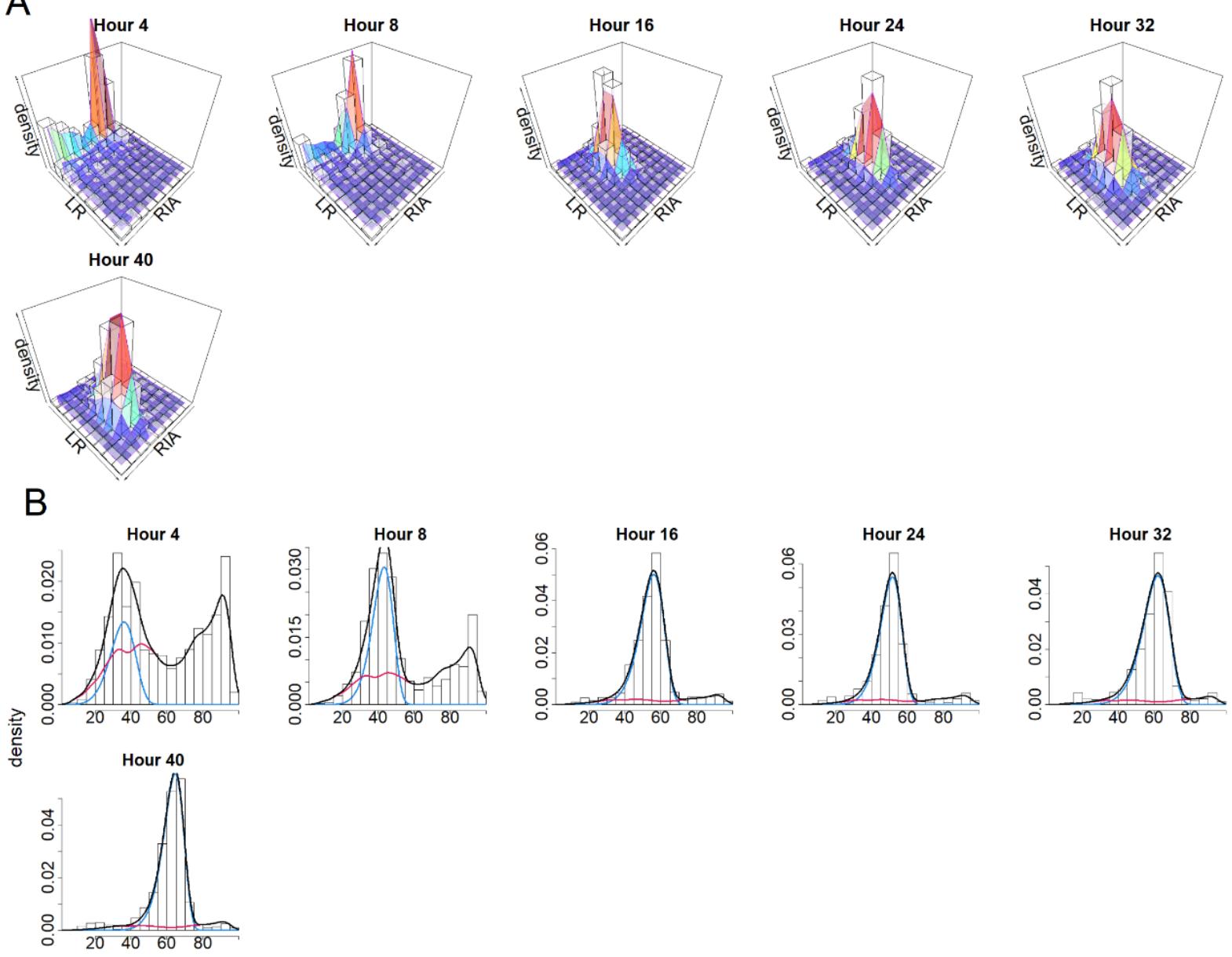

C
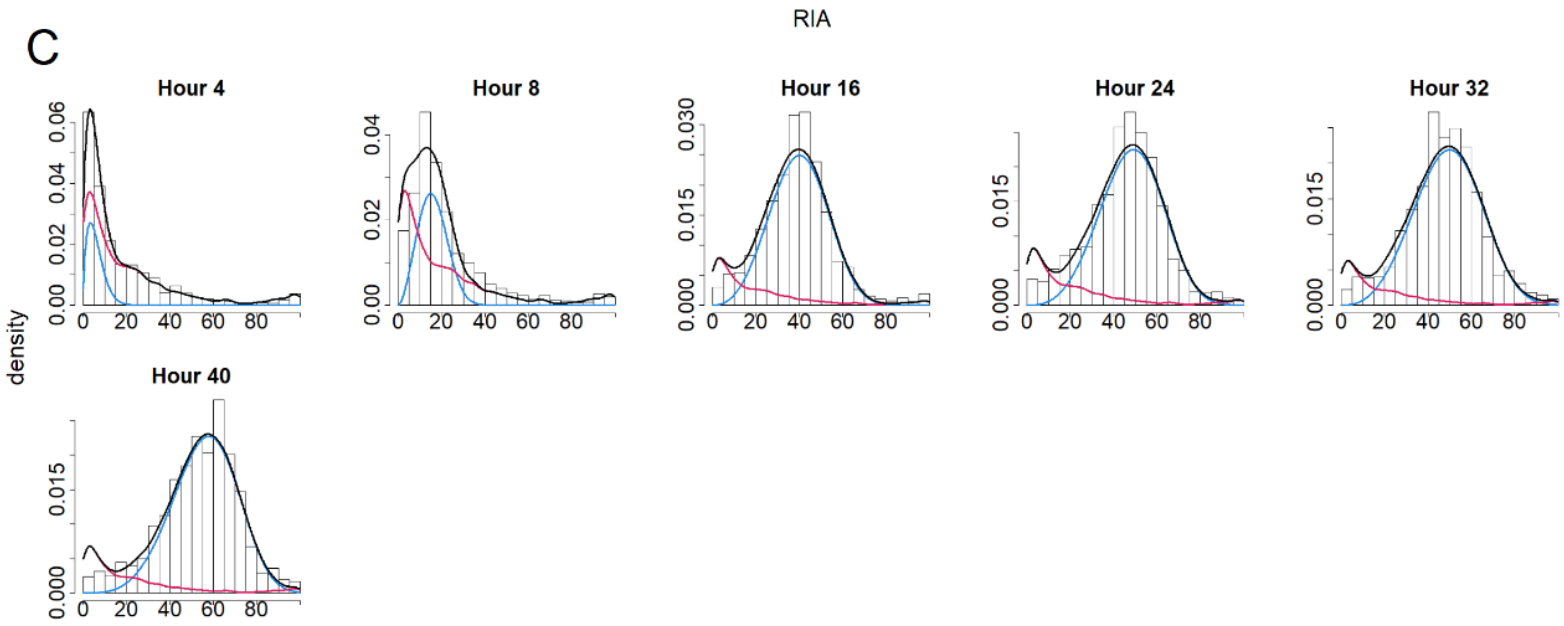
Figure 3.12: LR and RIA Distribution of Mixture, False, and True discoveries for the Soluble Fraction Sample. A) A bar plot representing the histogram of RIA and LR at each time point. The surface plot is the estimated joint density distribution calculated from the maximum likelihood estimate. B) and C) are the marginals from the of joint distribution where false discoveries is in red, mixture is in black, and true discoveries is in blue. 
A
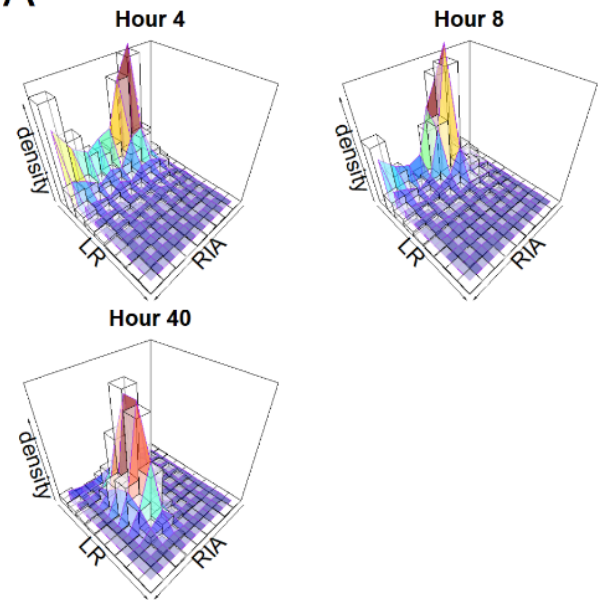

B

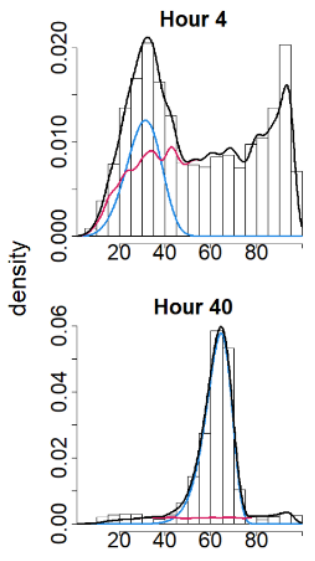

C
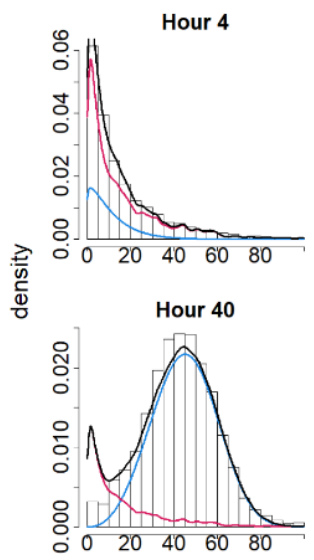
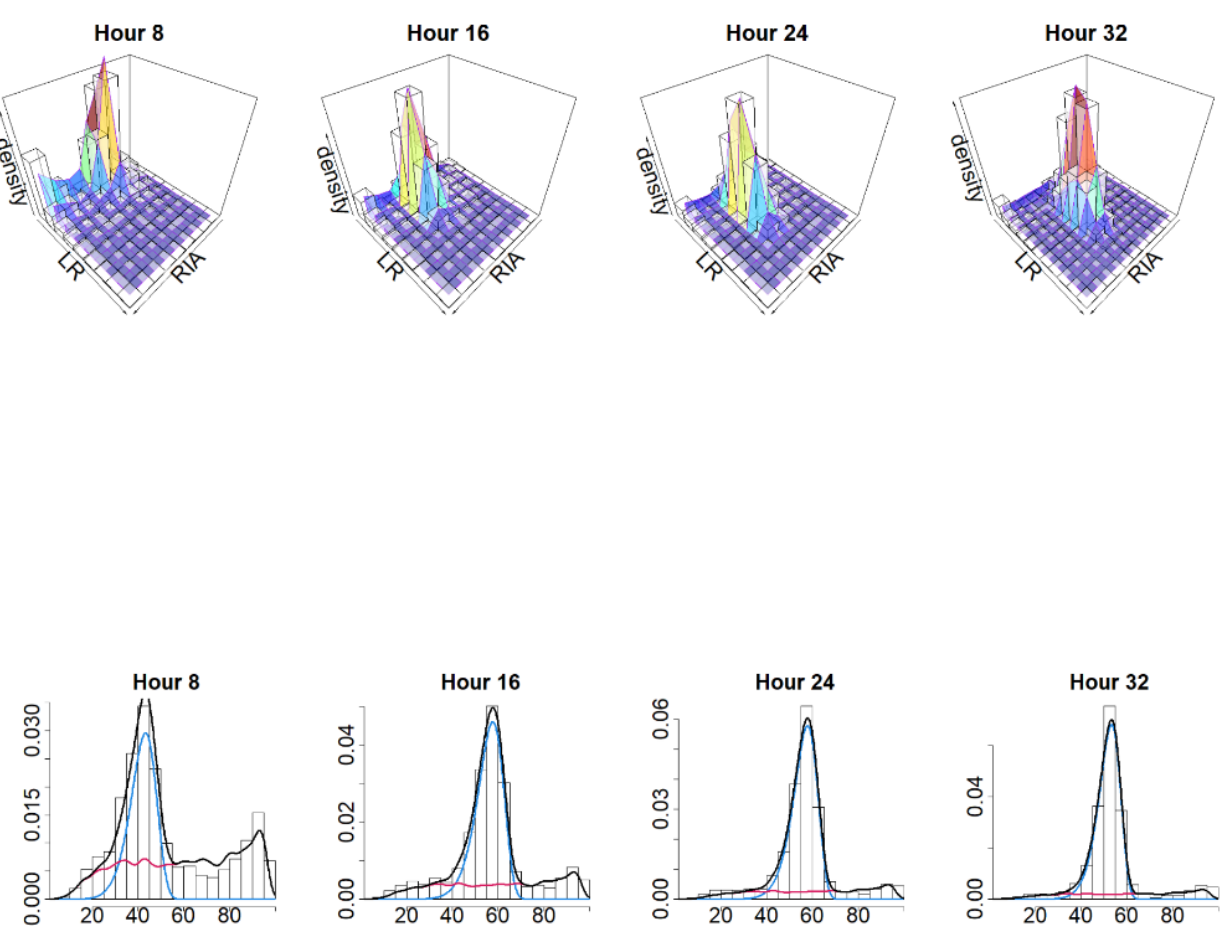

RIA
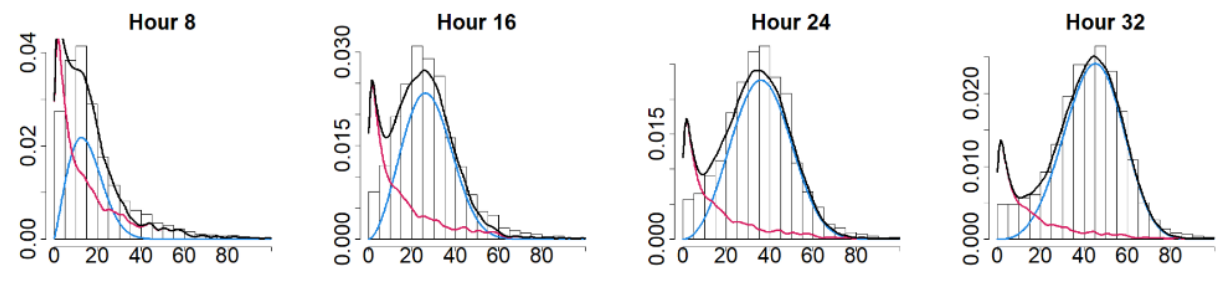
Figure 3.13: LR and RIA Distribution of Mixture, False, and True discoveries for the Microsomal Fraction Sample. A) A bar plot representing the histogram of RIA and LR at each time point. The surface plot is the estimated joint density distribution calculated from the maximum likelihood estimate. B) and C) are the marginals from the of joint distribution of RIA and LR where false discoveries is in red, mixture is in black, and true discoveries is in blue. 
A
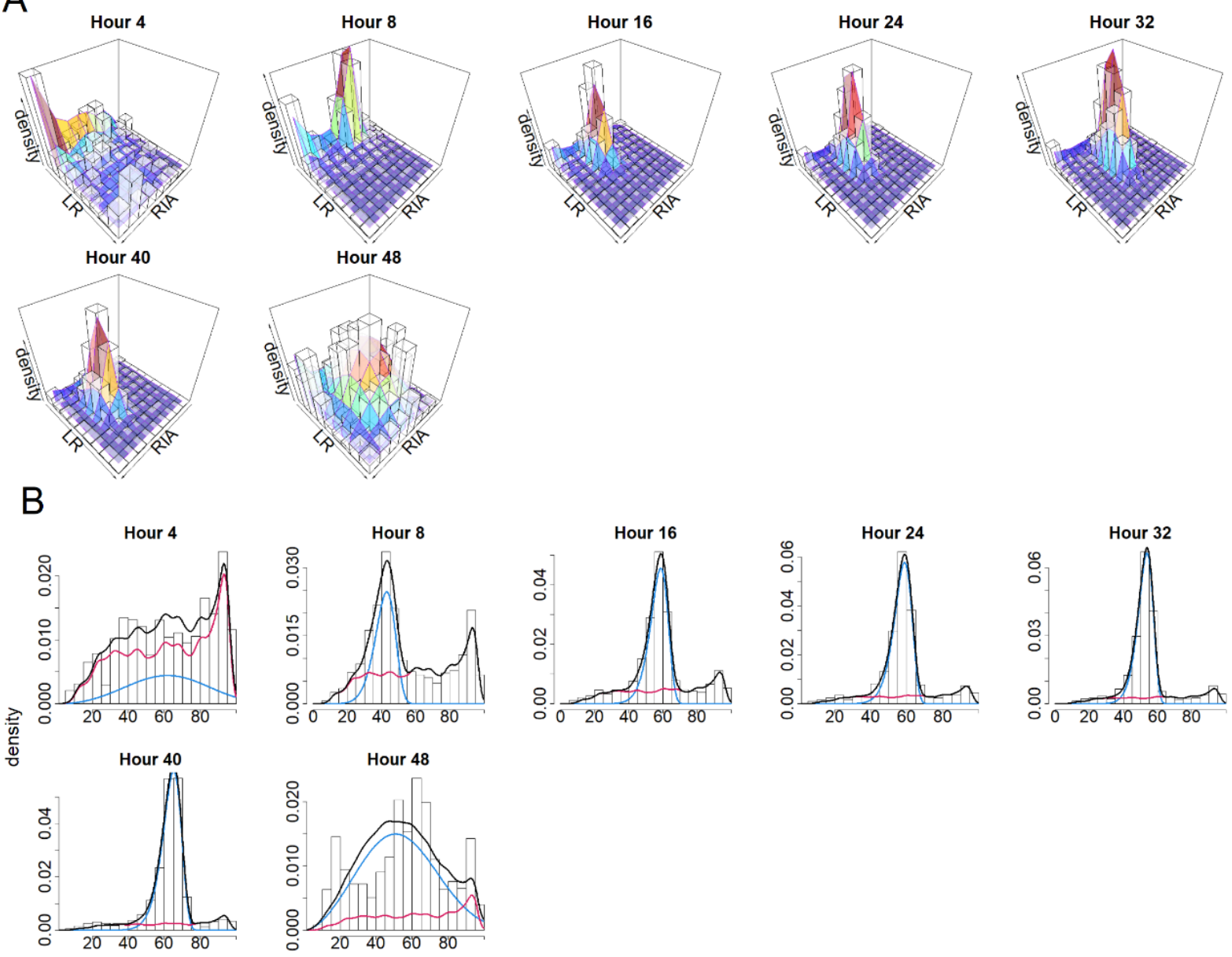

C
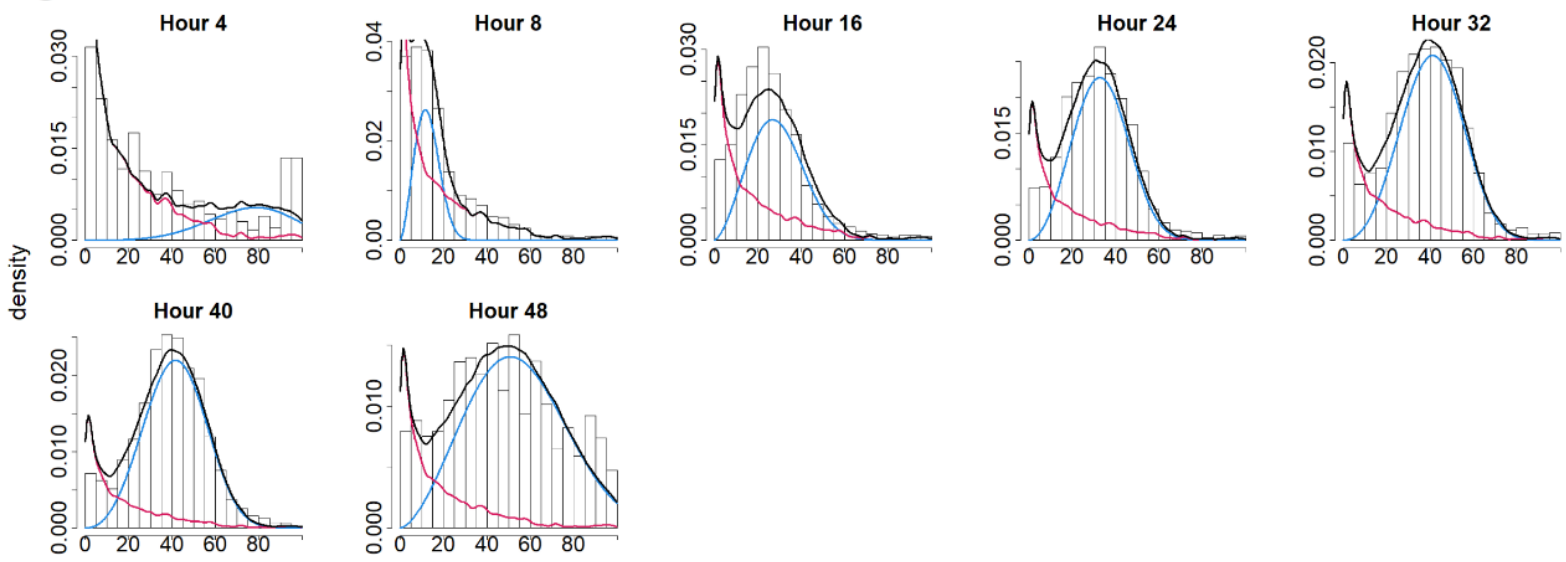
Figure 3.14: LR and RIA Distribution of Mixture, False, and True discoveries for the Organellar Fraction Sample. A) A bar plot representing the histogram of RIA and LR at each time point. The surface plot is the estimated joint density distribution calculated from the maximum likelihood estimate. B) and C) are the marginals from the of joint distribution where false discoveries is in red, mixture is in black, and true discoveries is in blue. 


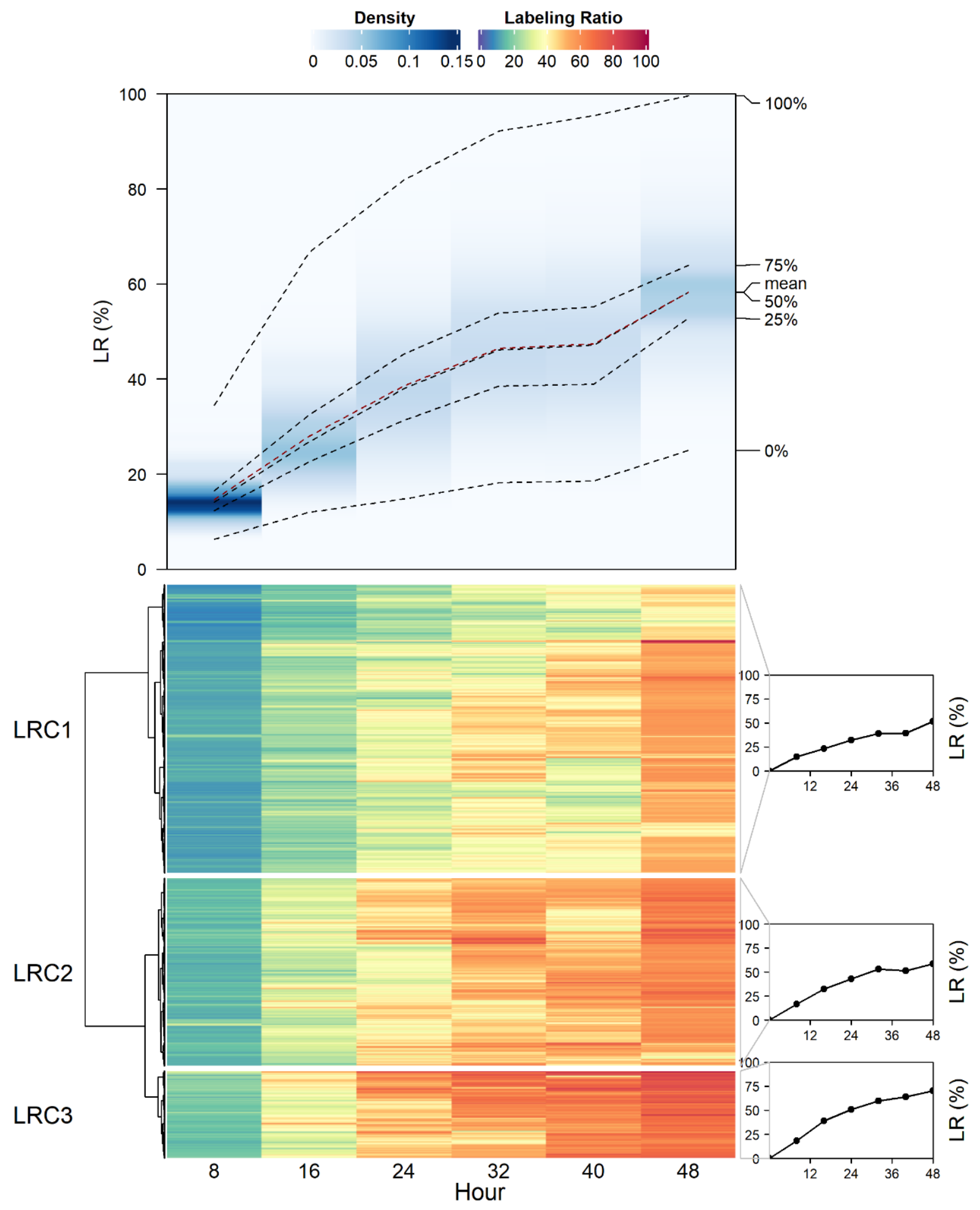


Figure 3.15: Isotope Incorporation Profile of Arabidopsis thaliana Seedling Roots. A) The top graph shows the distribution of RIA at each time point. Dark blue denotes a high density and light blue denotes a low density. At the bottom is a heatmap where the rows are peptides and the columns are the days at which the RIA was recorded. B) Similar to A except that it denotes relative intensity over time. Both heatmaps were clustered using Ward's minimum variance, as implemented in,74 and using the Euclidean distance. 
LRC2, and LRC3), where cluster LRC1 had the lowest rate. Hypergeometric tests showed that the organellar fraction is significantly over-represented in this cluster $(\mathrm{p}$-value $=1 \times$ $10^{-7}$; BH procedure). Peptides from cluster LRC3 displayed the highest rate from the three clusters and hypergeometric tests showed that the soluble fraction sample is overrepresented in LRC3 ( $\mathrm{p}$-value $=4 \times 10^{-9} ; \mathrm{BH}$ procedure). As for LRC2, the microsomal fraction is significantly over-represented in this cluster ( $\mathrm{p}$-value $=1 \times 10^{-7} ; \mathrm{BH}$ procedure) These results are all in agreement with Fan et al. (2016).

Of the 251 proteins analyzed in Fan et al. (2016) 248 proteins were observed from the results generated by our workflow. The coefficient of variation of the RMSD between the $\log 2(\mathrm{k})$ values of the two sets of results is $7 \%$ and the Pearson correlation coefficient is 0.84 . In addition to these proteins, the incorporation of 743 proteins were also analyzed, making a total of 991 unique proteins, The fitted curves for the three samples is shown in Figure 3.16A, as well as the fitted curves for the 12 proteins with the most peptides associated to them (Figure 3.16B-M). 

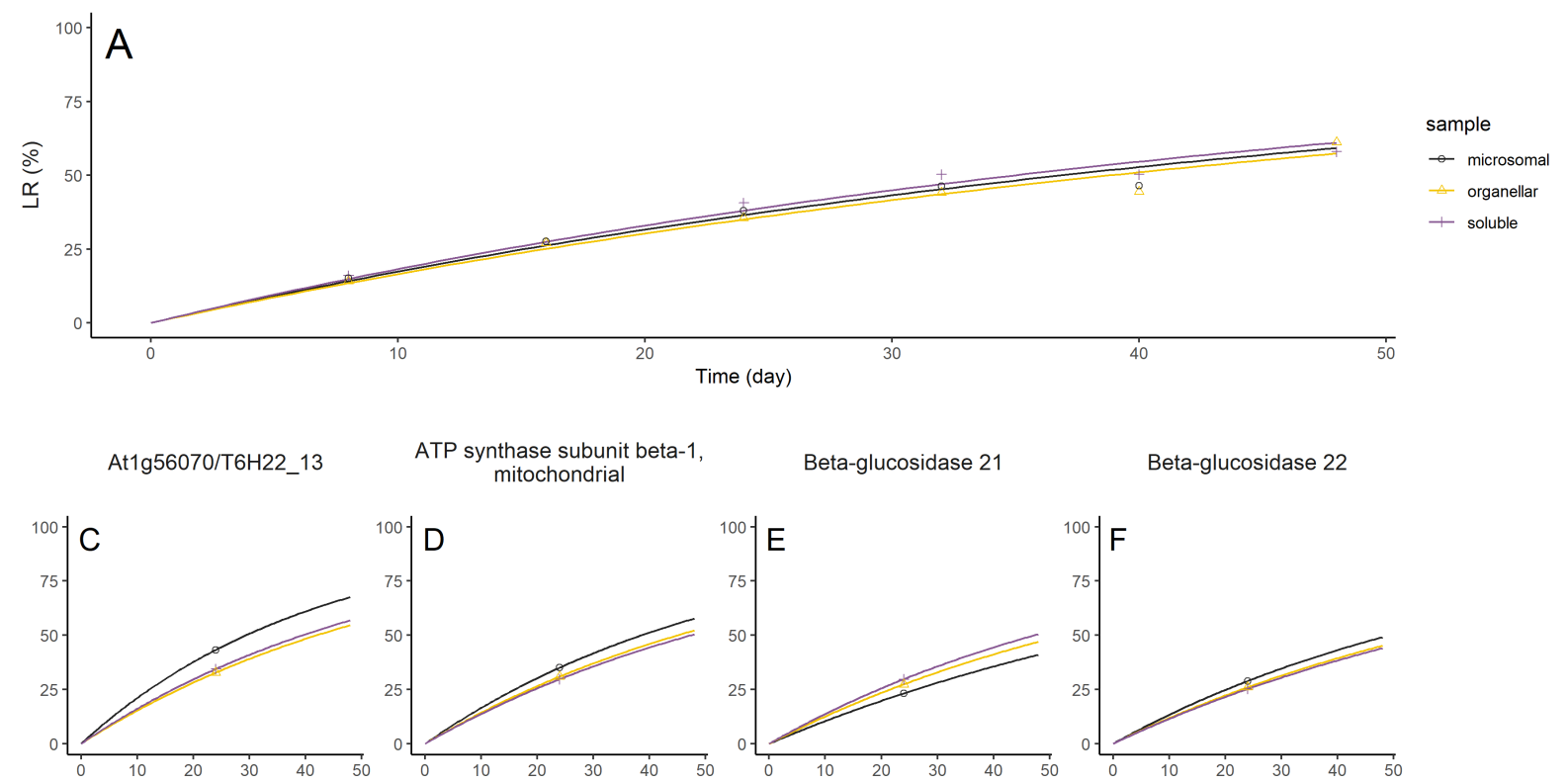

ATP synthase subunit beta-1, mitochondrial

Beta-glucosidase 21

Beta-glucosidase 22
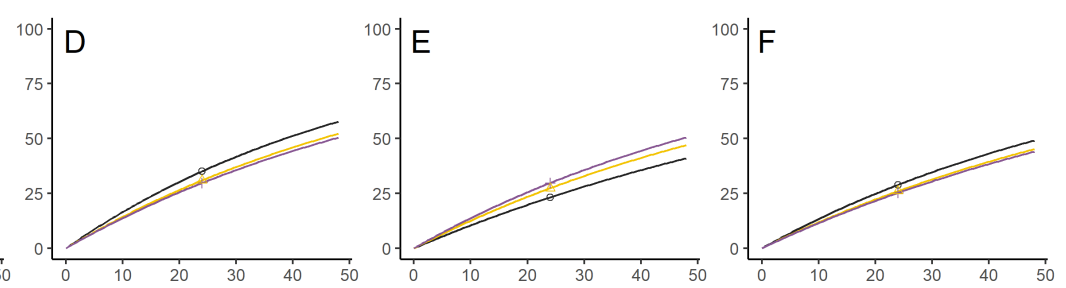

Beta-glucosidase 23

Bifunctional enolase 2/transcriptional activator

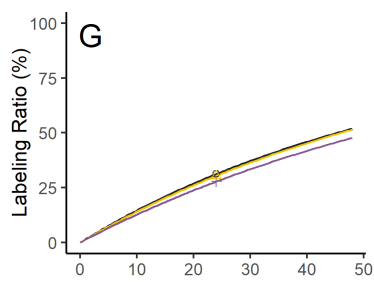

$\mathrm{NADH}$ dehydrogenase [ubiquinone] 1 alpha subcomplex subunit 9

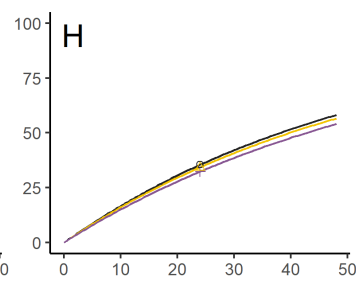

Probable mediator of RNA polymerase II transcription mitochondrial

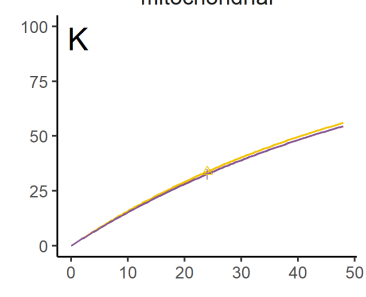
subunit $37 e$

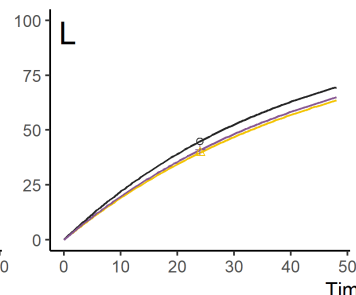

GDSL esterase/lipase At1g54000

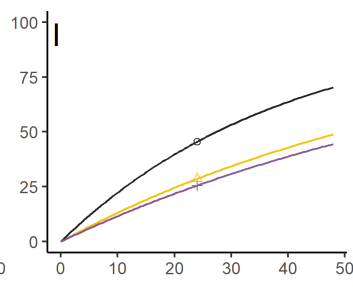

PYK10-binding protein 1

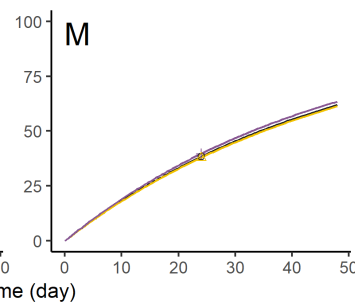

Jacalin-like lectin domain-containing protein

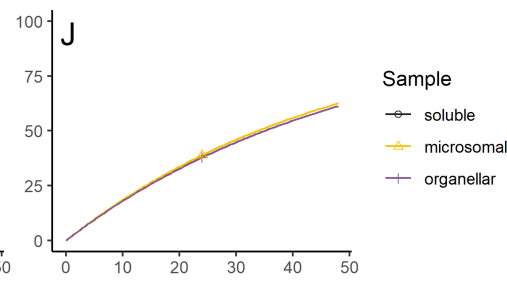

V-type proton ATPase

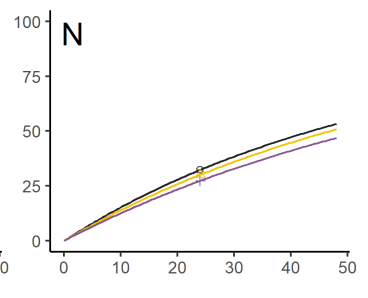


Figure 3.16: LR Profiles Over Time. (A) the LR profiles for each sample; (B-M) Average sample LR profiles over time for the 12 proteins with the most peptides associated to them 
A
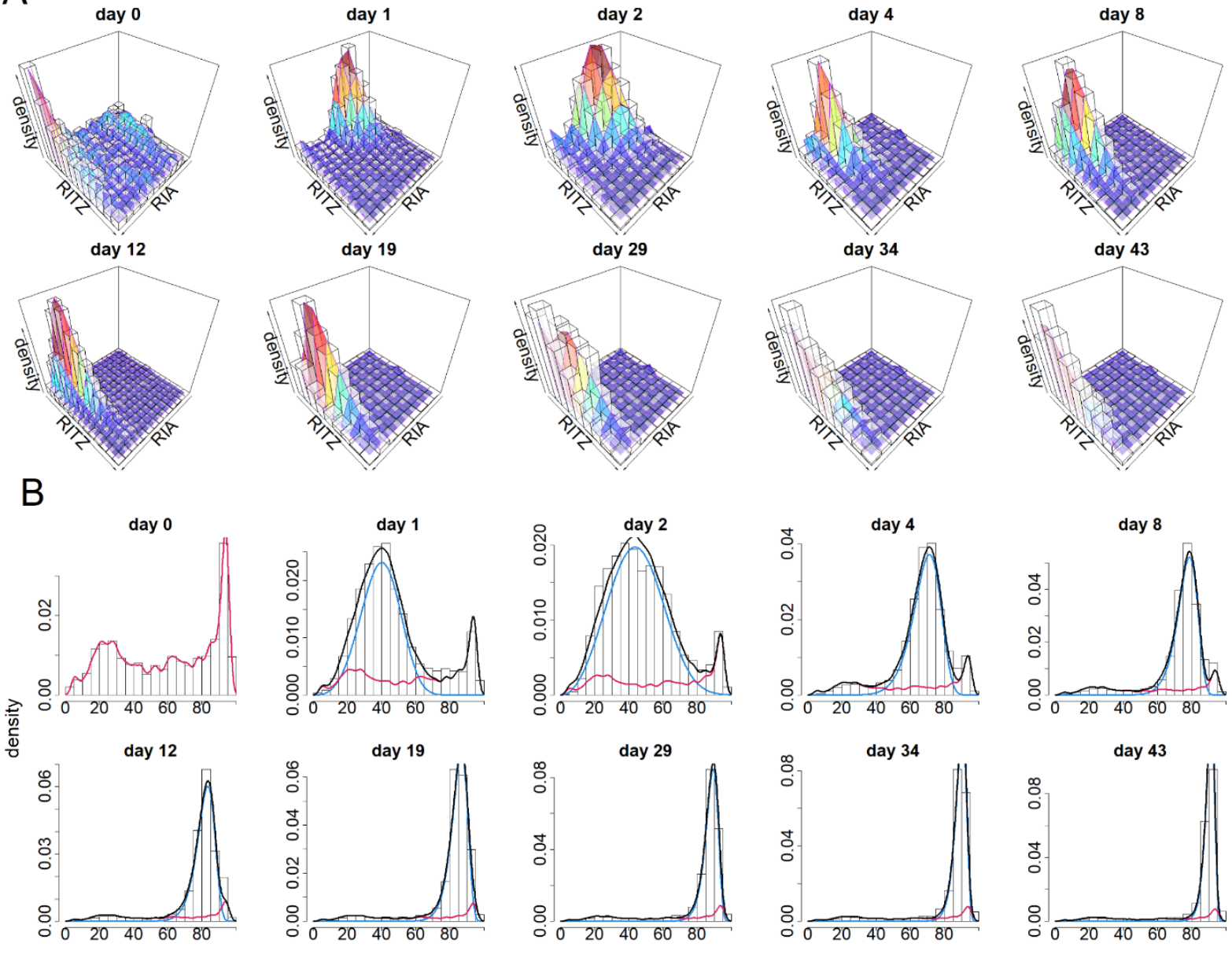

C
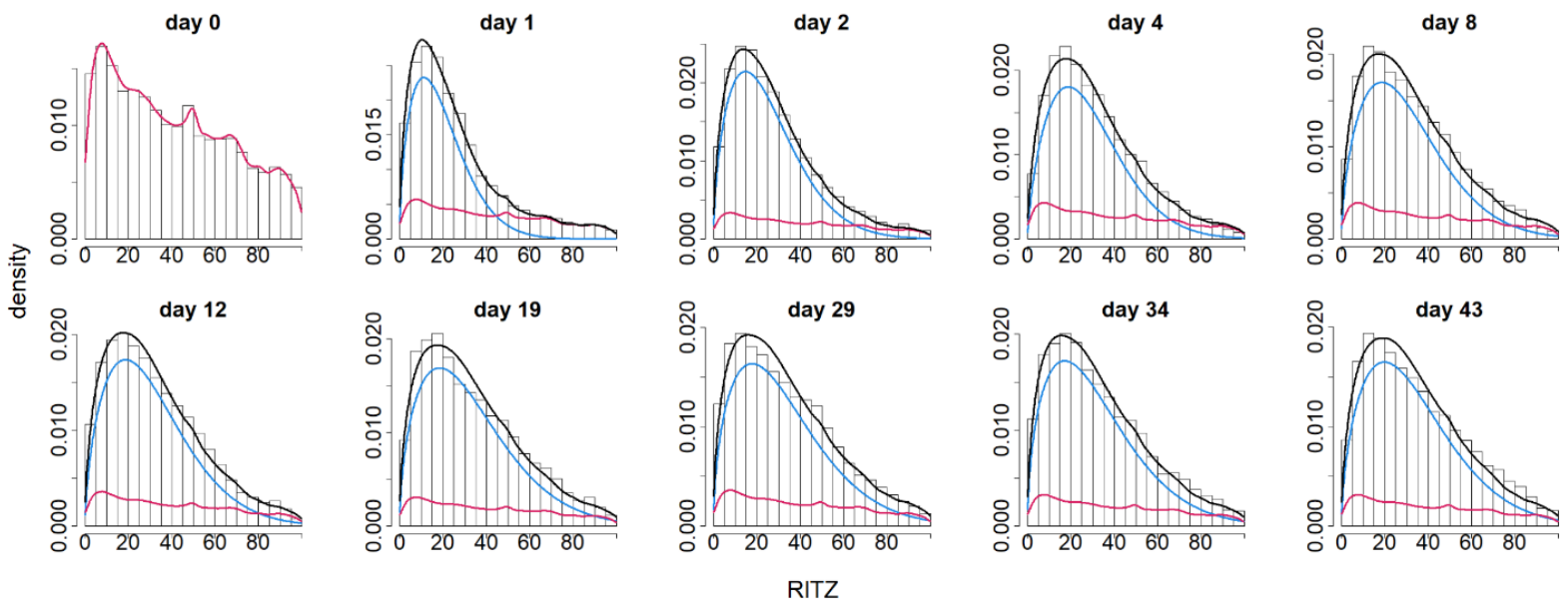
Figure 3.17: RITZ and RIA Distribution of Mixture, False, and True discoveries. A) A bar plot representing the histogram of RIA and RITZ at each time point. The surface plot is the estimated joint density distribution calculated from the maximum likelihood estimate. B) and C) are the marginals from the of joint distribution where false discoveries is in red, mixture is in black, and true discoveries is in blue. 


\section{Chapter 4}

\section{Discussion}

I investigated the in vivo dynamics of the microbiome using metaproteomics coupled with protein-SIP. Our experiment differs from previous protein-sip studies(Oberbach et al., 2017; Fan et al., 2016; Arike et al., 2020) by the addition of a protein spike-in to our

labeled sample. One disadvantage of a protein spike-in is that it would mask the rate of disappearance of the light peptide(Sachsenberg et al., 2015), thus making protein turnover estimation difficult. However, it is arguable that this approach can be used to measure protein turnover in the gut microbiome. Many factors such as elimination, de novo synthesis, protein recycling, etc will influence the observed abundance of the light and heavy peptide. Also, without a spike-in, very few peptides are observed as demonstrated by Oberbach et al. (2017). Thus, since our experiment mostly concerns with the general activity of the microbiome, I instead estimated the rate of newly synthesized (i.e. heavy) peptides by using the light peptide as a form of internal standard.

One challenge when dealing with ${ }^{15} \mathrm{~N}$ labeled proteomic data, from the microbiome in 
particular, is the difficulty in confidently identifying peptides from MS/MS spectra due to the partial incorporation of ${ }^{15} \mathrm{~N}$ (Gajer et al., 2012; Egert et al., 2007). To address this issue, unlabeled microbiome samples (time $=0$ ) were spiked in every partially labeled sample to ensure the presence of the corresponding reference light peptide. This allowed to achieve a greater number of peptides identified than the previous research by Oberbach et al. (2017).

Sachsenberg et al. (2015) were the first to introduce confidence assessment in proteinSIP experiments. In their paper, they estimated the quality of the results by calculating false positive rate (FPR) using contaminants or unlabeled reference samples. FPR is the expected proportion of all the true false positives in a study population selected as hits, whereas FDR is the expected proportion of true false positives among declared hits. The number of true false positives in a study population is typically unknown, whereas the number of declared as hits is known. Therefore, FPR is not typically verifiable and of less interest relative to FDR, which is verifiable. One can similarly use the scores from the contaminants and the scores from the observed values to estimate FDR. Unfortunately, when testing for hundreds or thousands of hypotheses, the distinction between true and false discoveries become blurred in an overall false-discovery rate(Verheggen et al., 2020; Zhang et al., 2017). An empirical Bayes model, on the other hand, allows for an error rate to be attributed for each selected feature. Thus, the strength of the evidence between each test is not lost in local false discovery rate. Another advantage is that LFDR can use multiple parameters, which increases power(Korthauer et al., 2019).

Since the median LFDR for day 1, 2, 4, 8, 12, 19, 29, 34, and 43 are $11.81 \%, 8.7 \%$, $5.64 \%, 4.71 \%, 4.67 \%, 4.37 \%, 5.48 \%, 4.8 \%$, and $4.61 \%$, respectively, the threshold was set 
at $10 \%$ so that earlier timepoints would not be filtered out completely. The reason for LFDR being higher in earlier timepoints is because low RIAs are more likely to be false as MetaProSIP may sometimes mistake the light peptide feature as being overlapped with the heavy peptide feature at time zero. This is why a higher density of RIA is observed for false discoveries in earlier timepoints in Figure 3.17B. In addition, the quantified features that are the result of experimental noise will often be low in RITZ as seen in Figure 3.17B.

Plateau detection was done using a rolling standard deviation, where a window of fixed length moves over the RITZ of each peptide and then computes the standard deviation of the RITZ within the window. Since windows with larger start to end gaps will tend to have smaller deviations and at least two observations are needed to compute standard deviation, nine days were chosen as the length. This span has the smallest root mean square deviation between the width of each window, compared to other spans. The Wilcoxon tests suggest that the plateau starts at day 29 and the RITZs past this day reflects the maximum proportion of the labeled proteins that the taxon/function can attain when using hydrolysate as a nitrogen source. It is important to note that several studies have reported that ${ }^{15} \mathrm{~N}$ metabolic labeling can effect certain cells, causing certain genes to be under or over expressed.(Filiou et al., 2012; Webhofer et al., 2013; Millard et al., 2015) To assess the contribution of the ${ }^{15} \mathrm{~N}$ isotope effect, I compared our dataset against the dataset by Webhofer et al.,(Webhofer et al., 2013) where they analysed blood plasma proteins from mice using the same diet as this study. However, the mice were fed in utero and for 56 days after birth, instead. The results from the comparison suggests that diet-induced compositional changes in microbiota may affect RITZ. In addition to the isotope effect 
from ${ }^{15} \mathrm{~N}$, factors such as nitrogen uptake, de novo synthesis, protein recycling, and internal storage will also affect heavy protein abundance in this study.

As shown in Figure 3.2, proteins from mice (Chordata) were within the highest peptide relative intensities $(0.572 \pm 0.015$ at $95 \% \mathrm{CI})$. This is not particularly surprising as dietary proteins are the most common source of nitrogen for mammals. Firmicutes, on the other hand, consistently showed lower relative intensities. A possible explanation is that they do not process hydrolysate efficiently and may obtain their nitrogen source elsewhere, such as from fiber(Zhang et al., 2018a) or metabolites from other microorganism in the microbial community. Nitrogen fixation could also provide another source of unlabeled nitrogen; however, very few mammalian gut microbes are capable of nitrogen fixation(Berthold et al., 2009; Alka et al., 2019). It is more likely that the microbiota cells obtain light amino acids via host protein degradation, and de novo synthesis(Gajer et al., 2012). Overtime, the nitrogen pool will gradually grow towards heavy isotopes as the host increasingly incorporates the labeled heavy nitrogen. Lactic acid bacteria were notably the exception from the phyla Firmicutes as they are among the highest in RITZ. This suggests potential differences in nutritional mechanisms of lactic acid bacteria with other Firmicutes species. Functions of "carbohydrate transport and metabolism" and "amino acid transport and metabolism", "energy production and conversion", as well as "translation, ribosomal structure, and biogenesis" were among the categories with the highest number of heavy peptide associated to them. The composition of functions was similar to previous metaproteomic studies on the gut microbiome(Weisser and Choudhary, 2017), indicating that the labeling might not be biased to specific functional categories. 
The RITZ of proteins belonging to the functional group "amino acid transport and metabolism" corresponded well with the overall RITZ of heavy peptides in each taxon (Pearson's correlation coefficient $=0.80$ ). This makes sense as those which efficiently degrade hydrolysate will generally see a higher level of heavy peptides. However, there were a few taxa that displayed high overall levels of RITZ without having peptides belonging to this category. In particular, Akkermansia muciniphila, showed high RITZ in the functional group "carbohydrate transport and metabolism" (0.558 \pm 0.108 at 95\% CI; Figure 3.11C), which was consistent with its mucin degrading ability. Another taxon which showed this pattern is Lactobacillus $(0.595 \pm 0.048$ at $95 \% \mathrm{CI}$; Figure 3.11J). Although not as well characterized, several species of this lineage have been shown to possess proteins that degrade mucin(Chacón and Duong, 2018). In addition, both Akkermansia and Lactobacillus were high in "translation, ribosomal structure and biogenesis" $(0.667 \pm 0.081$ at $95 \% \mathrm{CI})$ and $0.462 \pm 0.150$ at $95 \% \mathrm{CI}$ ), respectively), which indicates that both are rapid growers.

By studying RIA over time, I analyzed the elemental flux of nitrogen in the gut and the results shows that its availability is consistent to most species. However, there were a few species and strains that showed a delay in nitrogen availability. In particular, certain strains of Parabacteroides goldsteinii had no increase in heavy nitrogen availability after day 1. Cells from the host also exhibited this pattern. However, once heavy nitrogen became readily available to them, these strains started to catch up with the earlier incorporators. When analyzing the RITZ over time, on the other hand, it was shown that Verrucomicrobia had a characteristic increasing trend and never fully reached a plateau over 43 days. This is also observed in the genus, Akkermansia (Figure 3.11C), for cate- 
gory "translation, ribosomal structure and biogenesis" and "carbohydrate transport and metabolism". A similar pattern was observed for Lactobacillus(Figure 3.11J) in these categories as well, which further provided support of their mucin degrading capabilities. It is possible that these taxa may play important roles in foraging nitrogen from the host to the microbiome. Thus, our findings may suggest that these taxa, especially A. muciniphila, are keystone species for the metabolic flux of nitrogen in the microbiome.

For this study I retained peptides that were not identified at time zero. It is possible that peptides identified at time zero were the result of MS/MS from heavy peptides being wrongfully assigned to a light peptide. One way that one can test the likelihood of these types of misassignments is by searching light peptides against a fully ${ }^{15} \mathrm{~N}$ labeled database. Since there were nine times more ${ }^{15} \mathrm{~N}$ peptides, I took the sample specific database generated from MetaLab and added randomly selected proteins from the large metaproteome database until the size became nine times larger than its original size. I then added ${ }^{15} \mathrm{~N}$ labeled proteins via fixed modifications and searched this database with the day zero samples. The resulting number of peptide identifications were seven at an FDR threshold of $1 \%$ (PSM level).

In the case that a heavy peptide would be assigned with a light peptide from MS/MS, they will likely get filtered out: 1) when removing results that do not have two RIAs: one below and above $\left.{ }^{15} N_{\max }{ }^{-1}, 2\right)$ when running MetaProSIP as peaks with a Pearson correlation lower than 0.6 are removed, and 3) when filtering via LFDR as they will likely have high local false from the distorted RIA and LR values.

I also investigated as to why some light peptides were not identified at time zero but 
identified in subsequent days. One possibility is that MS/MS were not generated at time zero for these peptides, which would be consistent with the results from Table 3.1. Namely, peptides identified in the subsequent days were too low to be selected for MS2 fragmentation at time zero, but due to having lower protein turnover, their peak intensities did not reduce as much as the other peptides and were able to be selected for MS2 fragmentation. This would also mean that, eventually, their intensities will become too low again to be selected for MS2 fragmentation. One can see this pattern in the number of peptide identifications as well (Table 3.2). where from day 1 to day 2, you see an increase in the number of ids for non-time zero peptides, while you get 2000 less ids for time zero peptides. Eventually, however, we get $\sim 50 \%$ less non-time zero peptide ids on day 43 than on day 1 , while for time zero, we get $30 \%$ less. This also explains why they were mostly found in RITZC1 due to their slow protein turnover. RIA was significantly lower as well for these peptides from day 4 to day 43 compared to peptides found at time zero (Table 3.3). 


\section{Chapter 5}

\section{Conclusions}

We investigated the in vivo dynamics of the microbiome using metaproteomics coupled with protein-SIP. Using dataset with light protein spike-in, I developped a new bioinformatics pipeline and a new bioinformatic tool called MetaProfiler which greatly enhanced the number of peptides identified $(15,297)$, and quantified $(10,173 ; 7,108$ with LFDR $<10 \%)$ with ${ }^{15} \mathrm{~N}$ incorporations. I used a novel approach to assess confidence of protein-SIP and I used machine learning algorithms to conduct the most in-depth analysis of mice gut microbiome dynamic study using protein-SIP, at the time of this study. I believe that the outcome of this study will allow more thorough research in microbiome dynamics using MetaProfiler and provide deeper insight into the functionality of the gut microbiome. 


\section{Chapter 6}

\section{Future Work}

One of the main limitations of using mouse models is that they cannot replicate human systems. Genetic variations, medical history, lifestyle choices, and so on, are important factors to consider in microbiome dynamics which are not taken into consideration when using mouse models. Therefore, future work in microbiome experiments using protein-SIP would need to incorporate in vitro human models. In addition, culturomics would eliminate factors that would affect light and heavy protein intensities over time such as elimination, de novo synthesis from neighboring species, host internal storage, and so on. Thus, we would be able to better understand the mechanisms affecting the proteome dynamics of the gut microbiome. While several studies are starting to look at the effects of therapeutic treatments on gut microbiome(Maier et al., 2018; Chen et al., 2017; Le Bastard et al., 2018; Li et al., 2020), no study so far has investigated the effects of these treatments on microbiome dynamics using protein-SIP. With the ever-increasing number of treatments, there is a pressing need in developing high-throughput methods to assess drug-microbiome 
dynamics interactions 


\section{References}

Aggarwal, K., Choe, L. H. and Lee, K. H. (2006). Shotgun proteomics using the iTRAQ isobaric tags. Briefings in Functional Genomics 5, 112-120.

Alka, O., Sachsenberg, T., Bichmann, L., Pfeuffer, J., Weisser, H., Wein, S., Netz, E., Rurik, M., Kohlbacher, O. and Rost, H. (2019). OpenMS for open source analysis of mass spectrometric data. PeerJ Preprints 7, e27766v1.

Arike, L., Seiman, A., van der Post, S., Piñeiro, A. M. R., Ermund, A., Schütte, A., Bäckhed, F., Johansson, M. E. and Hansson, G. C. (2020). Protein turnover in epithelial cells and mucus along the gastrointestinal tract is coordinated by the spatial location and microbiota. Cell reports 30, 1077-1087.

Asnicar, F., Weingart, G., Tickle, T. L., Huttenhower, C. and Segata, N. (2015). Compact graphical representation of phylogenetic data and metadata with GraPhlAn. PeerJ 3, e1029.

Berg, G., Rybakova, D., Fischer, D., Cernava, T., Vergès, M.-C. C., Charles, T., Chen, X., Cocolin, L., Eversole, K., Corral, G. H. et al. (2020). Microbiome definition re-visited: old concepts and new challenges. Microbiome 8, 1-22. 
Berry, D. and Loy, A. (2018). Stable-isotope probing of human and animal microbiome function. Trends in microbiology 26, 999-1007.

Berry, D., Stecher, B., Schintlmeister, A., Reichert, J., Brugiroux, S., Wild, B., Wanek, W., Richter, A., Rauch, I., Decker, T. et al. (2013). Host-compound foraging by intestinal microbiota revealed by single-cell stable isotope probing. Proceedings of the National Academy of Sciences 110, 4720-4725.

Berthold, M. R., Cebron, N., Dill, F., Gabriel, T. R., Kötter, T., Meinl, T., Ohl, P., Thiel, K. and Wiswedel, B. (2009). KNIME-the Konstanz information miner: version 2.0 and beyond. AcM SIGKDD explorations Newsletter 11, 26-31.

Caporaso, J. G., Lauber, C. L., Costello, E. K., Berg-Lyons, D., Gonzalez, A., Stombaugh, J., Knights, D., Gajer, P., Ravel, J., Fierer, N. et al. (2011). Moving pictures of the human microbiome. Genome biology 12, 1-8.

Chacón, J. E. and Duong, T. (2018). Multivariate kernel smoothing and its applications. CRC Press.

Charrad, M. and Ghazzali, N. (2014). Package 'nbclust'. Journal of statistical software $61,1-36$.

Chen, T., Li, J., Chen, T., Sun, C. C. and Zheng, Y. (2017). Tablets of multi-unit pellet system for controlled drug delivery. Journal of Controlled Release 262, 222-231.

Cheng, K., Ning, Z., Zhang, X., Li, L., Liao, B., Mayne, J., Stintzi, A. and Figeys, D. (2017). MetaLab: an automated pipeline for metaproteomic data analysis. Microbiome $5,1-10$. 
Conesa, A., Madrigal, P., Tarazona, S., Gomez-Cabrero, D., Cervera, A., McPherson, A., Szcześniak, M. W., Gaffney, D. J., Elo, L. L., Zhang, X. et al. (2016). A survey of best practices for RNA-seq data analysis. Genome biology 17, 13.

Cox, J. and Mann, M. (2008). MaxQuant enables high peptide identification rates, individualized p.p.b.-range mass accuracies and proteome-wide protein quantification. Nat Biotechnol 26, 1367-72.

Cox, J., Neuhauser, N., Michalski, A., Scheltema, R. A., Olsen, J. V. and Mann, M. (2011). Andromeda: a peptide search engine integrated into the MaxQuant environment. Journal of proteome research $10,1794-1805$.

Eddelbuettel, D. (2010). RcppDE: Global optimization by differential evolution in C++. R package version 0.1. 0, URL http://CRAN.R-project.org/package=RcppDE .

Efron, B., Tibshirani, R., Storey, J. D. and Tusher, V. (2001). Empirical Bayes analysis of a microarray experiment. Journal of the American statistical association 96, 1151-1160.

Egert, M., De Graaf, A. A., Maathuis, A., De Waard, P., Plugge, C. M., Smidt, H., Deutz, N. E., Dijkema, C., De Vos, W. M. and Venema, K. (2007). Identification of glucosefermenting bacteria present in an in vitro model of the human intestine by RNA-stable isotope probing. FEMS microbiology ecology 60, 126-135.

Eng, J. K., McCormack, A. L. and Yates, J. R. (1994). An approach to correlate tandem mass spectral data of peptides with amino acid sequences in a protein database. Journal of the american society for mass spectrometry $\quad 5,976-989$. 
Fan, K.-T., Rendahl, A. K., Chen, W.-P., Freund, D. M., Gray, W. M., Cohen, J. D. and Hegeman, A. D. (2016). Proteome scale-protein turnover analysis using high resolution mass spectrometric data from stable-isotope labeled plants. Journal of proteome research $15,851-867$.

Filiou, M. D., Varadarajulu, J., Teplytska, L., Reckow, S., Maccarrone, G. and Turck, C. W. (2012). The $15 \mathrm{~N}$ isotope effect in Escherichia coli: A neutron can make the difference. Proteomics 12, 3121-3128.

Flores, G. E., Caporaso, J. G., Henley, J. B., Rideout, J. R., Domogala, D., Chase, J., Leff, J. W., Vázquez-Baeza, Y., Gonzalez, A., Knight, R. et al. (2014). Temporal variability is a personalized feature of the human microbiome. Genome biology 15,531 .

Franzosa, E. A., Sirota-Madi, A., Avila-Pacheco, J., Fornelos, N., Haiser, H. J., Reinker, S., Vatanen, T., Hall, A. B., Mallick, H., McIver, L. J., Sauk, J. S., Wilson, R. G., Stevens, B. W., Scott, J. M., Pierce, K., Deik, A. A., Bullock, K., Imhann, F., Porter, J. A., Zhernakova, A., Fu, J., Weersma, R. K., Wijmenga, C., Clish, C. B., Vlamakis, H., Huttenhower, C. and Xavier, R. J. (2019). Gut microbiome structure and metabolic activity in inflammatory bowel disease. Nat Microbiol 4, 293-305.

Gajer, P., Brotman, R. M., Bai, G., Sakamoto, J., Schütte, U. M., Zhong, X., Koenig, S. S., Fu, L., Ma, Z. S., Zhou, X. et al. (2012). Temporal dynamics of the human vaginal microbiota. Science translational medicine 4, 132ra52-132ra52.

Gu, Z., Eils, R. and Schlesner, M. (2016). Complex heatmaps reveal patterns and correlations in multidimensional genomic data. Bioinformatics 32, 2847-2849. 
Guan, S., Price, J. C., Ghaemmaghami, S., Prusiner, S. B. and Burlingame, A. L. (2012). Compartment modeling for mammalian protein turnover studies by stable isotope metabolic labeling. Analytical chemistry 84, 4014-4021.

Heintz-Buschart, A., May, P., Laczny, C. C., Lebrun, L. A., Bellora, C., Krishna, A., Wampach, L., Schneider, J. G., Hogan, A., De Beaufort, C. et al. (2016). Integrated multi-omics of the human gut microbiome in a case study of familial type 1 diabetes. Nature microbiology 2, 1-13.

Heintz-Buschart, A. and Wilmes, P. (2018). Human gut microbiome: function matters. Trends in microbiology 26, 563-574.

Helmink, B. A., Khan, M. A. W., Hermann, A., Gopalakrishnan, V. and Wargo, J. A. (2019). The microbiome, cancer, and cancer therapy. Nat Med 25, 377-388.

Herrmann, E., Young, W., Rosendale, D., Conrad, R., Riedel, C. U. and Egert, M. (2017a). Determination of resistant starch assimilating bacteria in fecal samples of mice by in vitro RNA-based stable isotope probing. Frontiers in microbiology 8, 1331.

Herrmann, E., Young, W., Rosendale, D., Reichert-Grimm, V., Riedel, C. U., Conrad, R. and Egert, M. (2017b). RNA-based stable isotope probing suggests Allobaculum spp. as particularly active glucose assimilators in a complex murine microbiota cultured in vitro. BioMed Research International $201 \%$.

Jagtap, P. D., Blakely, A., Murray, K., Stewart, S., Kooren, J., Johnson, J. E., Rhodus, N. L., Rudney, J. and Griffin, T. J. (2015). Metaproteomic analysis using the Galaxy framework. Proteomics 15, 3553-3565. 
Jehmlich, N., Vogt, C., Lunsmann, V., Richnow, H. H. and von Bergen, M. (2016). ProteinSIP in environmental studies. Curr Opin Biotechnol 41, 26-33.

Kim, H. J., Li, H., Collins, J. J. and Ingber, D. E. (2016). Contributions of microbiome and mechanical deformation to intestinal bacterial overgrowth and inflammation in a human gut-on-a-chip. Proceedings of the National Academy of Sciences 113, E7-E15.

Klaassen, C. D. and Aleksunes, L. M. (2010). Xenobiotic, bile acid, and cholesterol transporters: function and regulation. Pharmacol Rev 62, $18-23$.

Komaroff, A. L. (2017). The Microbiome and Risk for Obesity and Diabetes. JAMA 317, $355-356$.

Korthauer, K., Kimes, P. K., Duvallet, C., Reyes, A., Subramanian, A., Teng, M., Shukla, C., Alm, E. J. and Hicks, S. C. (2019). A practical guide to methods controlling false discoveries in computational biology. Genome biology 20, 1-21.

Kovatcheva-Datchary, P. and Egert, M. (2009). M., M. Rajilić-Stojanović, A. de Graaf, H. Smidt, W. de Vos and K. Venema, Linking phylogenetic identities of bacteria to starch fermentation in an in vitro model of the large intestine by RNA-based stable isotope probing. Environ. Microbiol 11, 914-926.

Lagier, J.-C., Dubourg, G., Million, M., Cadoret, F., Bilen, M., Fenollar, F., Levasseur, A., Rolain, J.-M., Fournier, P.-E. and Raoult, D. (2018). Culturing the human microbiota and culturomics. Nature Reviews Microbiology 16, 540-550.

Lavelle, A. and Sokol, H. (2018). Gut microbiota: beyond metagenomics, metatranscrip- 
tomics illuminates microbiome functionality in IBD. Nature Reviews Gastroenterology \& Hepatology 15, 193-194.

Le Bastard, Q., Al-Ghalith, G., Grégoire, M., Chapelet, G., Javaudin, F., Dailly, E., Batard, E., Knights, D. and Montassier, E. (2018). Systematic review: human gut dysbiosis induced by non-antibiotic prescription medications. Alimentary Pharmacology \& Therapeutics 47, 332-345.

Li, L., Ning, Z., Zhang, X., Mayne, J., Cheng, K., Stintzi, A. and Figeys, D. (2020). RapidAIM: A culture-and metaproteomics-based Rapid Assay of Individual Microbiome responses to drugs. Microbiome 8,1-16.

Liu, C., Song, C.-Q., Yuan, Z.-F., Fu, Y., Chi, H., Wang, L.-H., Fan, S.-B., Zhang, K., Zeng, W.-F., He, S.-M. et al. (2014). pQuant improves quantitation by keeping out interfering signals and evaluating the accuracy of calculated ratios. Analytical chemistry $86,5286-5294$.

Livanos, A., Greiner, T., Vangay, P., Pathmasiri, W., Stewart, D., McRitchie, S., Li, H., Chung, J., Sohn, J., Kim, S. et al. (2016). Antibiotic-mediated gut microbiome perturbation accelerates development of type 1 diabetes in mice. Nat Microbiol 1: 16140.

Maier, L., Pruteanu, M., Kuhn, M., Zeller, G., Telzerow, A., Anderson, E. E., Brochado, A. R., Fernandez, K. C., Dose, H., Mori, H. et al. (2018). Extensive impact of nonantibiotic drugs on human gut bacteria. Nature 555, 623-628.

Maruvada, P., Leone, V., Kaplan, L. M. and Chang, E. B. (2017). The Human Microbiome and Obesity: Moving beyond Associations. Cell Host Microbe 22, 589-599. 
McClure, R., Balasubramanian, D., Sun, Y., Bobrovskyy, M., Sumby, P., Genco, C. A., Vanderpool, C. K. and Tjaden, B. (2013). Computational analysis of bacterial RNA-Seq data. Nucleic acids research 41, e140-e140.

McDonald, J. A., Fuentes, S., Schroeter, K., Heikamp-deJong, I., Khursigara, C. M., de Vos, W. M. and Allen-Vercoe, E. (2015). Simulating distal gut mucosal and luminal communities using packed-column biofilm reactors and an in vitro chemostat model. Journal of microbiological methods 108, 36-44.

Millard, P., Portais, J.-C. and Mendes, P. (2015). Impact of kinetic isotope effects in isotopic studies of metabolic systems. BMC systems biology 9, 1-13.

Mottino, A. D., Hoffman, T., Jennes, L. and Vore, M. (2000). Expression and localization of multidrug resistant protein mrp2 in rat small intestine. J Pharmacol Exp Ther 293, $717-23$.

Muth, T., Kohrs, F., Heyer, R., Benndorf, D., Rapp, E., Reichl, U., Martens, L. and Renard, B. Y. (2018). MPA portable: a stand-alone software package for analyzing metaproteome samples on the go. Analytical chemistry 90, 685-689.

Oberbach, A., Haange, S. B., Schlichting, N., Heinrich, M., Lehmann, S., Till, H., Hugenholtz, F., Kullnick, Y., Smidt, H., Frank, K., Seifert, J., Jehmlich, N. and von Bergen, M. (2017). Metabolic in Vivo Labeling Highlights Differences of Metabolically Active Microbes from the Mucosal Gastrointestinal Microbiome between High-Fat and Normal Chow Diet. J Proteome Res 16, 1593-1604.

Olsen, J. V., Macek, B., Lange, O., Makarov, A., Horning, S. and Mann, M. (2007). 
Higher-energy C-trap dissociation for peptide modification analysis. Nature methods 4 , 709-712.

Park, S. K. R., Aslanian, A., McClatchy, D. B., Han, X., Shah, H., Singh, M., Rauniyar, N., Moresco, J. J., Pinto, A. F., Diedrich, J. K. et al. (2014). Census 2: isobaric labeling data analysis. Bioinformatics 30, 2208-2209.

Pedrioli, P. G. (2010). Trans-proteomic pipeline: a pipeline for proteomic analysis. In Proteome Bioinformatics pp. 213-238. Springer.

Perkins, D. N., Pappin, D. J., Creasy, D. M. and Cottrell, J. S. (1999). Probability-based protein identification by searching sequence databases using mass spectrometry data. ELECTROPHORESIS: An International Journal 20,3551-3567.

Price, J. C., Guan, S., Burlingame, A., Prusiner, S. B. and Ghaemmaghami, S. (2010). Analysis of proteome dynamics in the mouse brain. Proceedings of the National Academy of Sciences 107, 14508-14513.

Ralls, M. W., Demehri, F. R., Feng, Y., Raskind, S., Ruan, C., Schintlmeister, A., Loy, A., Hanson, B., Berry, D., Burant, C. F. et al. (2016). Bacterial nutrient foraging in a mouse model of enteral nutrient deprivation: insight into the gut origin of sepsis. American Journal of Physiology-Gastrointestinal and Liver Physiology 311, G734-G743.

Rauniyar, N., McClatchy, D. B. and Yates III, J. R. (2013). Stable isotope labeling of mammals (SILAM) for in vivo quantitative proteomic analysis. Methods 61, 260-268.

Röst, H. L., Sachsenberg, T., Aiche, S., Bielow, C., Weisser, H., Aicheler, F., Andreotti, S., Ehrlich, H.-C., Gutenbrunner, P., Kenar, E. et al. (2016). OpenMS: a flexible open- 
source software platform for mass spectrometry data analysis. Nature methods 13, 741.

Rowland, I., Gibson, G., Heinken, A., Scott, K., Swann, J., Thiele, I. and Tuohy, K. (2018). Gut microbiota functions: metabolism of nutrients and other food components. European journal of nutrition 57, 1-24.

Sachsenberg, T., Herbst, F.-A., Taubert, M., Kermer, R., Jehmlich, N., von Bergen, M., Seifert, J. and Kohlbacher, O. (2015). MetaProSIP: automated inference of stable isotope incorporation rates in proteins for functional metaproteomics. Journal of proteome research 14, 619-627.

Sato, T. and Clevers, H. (2013). Growing self-organizing mini-guts from a single intestinal stem cell: mechanism and applications. Science 340, 1190-1194.

Senko, M. W., Beu, S. C. and McLaffertycor, F. W. (1995). Determination of monoisotopic masses and ion populations for large biomolecules from resolved isotopic distributions. Journal of the American Society for Mass Spectrometry 6, 229-233.

Shafin, K., Pesout, T., Lorig-Roach, R., Haukness, M., Olsen, H. E., Bosworth, C., Armstrong, J., Tigyi, K., Maurer, N., Koren, S. et al. (2020). Nanopore sequencing and the Shasta toolkit enable efficient de novo assembly of eleven human genomes. Nature Biotechnology ., 1-10.

Sleno, L. and Volmer, D. A. (2004). Ion activation methods for tandem mass spectrometry. Journal of mass spectrometry 39, 1091-1112. 
Slysz, G. W., Steinke, L., Ward, D. M., Klatt, C. G., Clauss, T. R., Purvine, S. O., Payne, S. H., Anderson, G. A., Smith, R. D. and Lipton, M. S. (2014). Automated data extraction from in situ protein-stable isotope probing studies. Journal of proteome research 13, 1200-1210.

Starr, A. E., Deeke, S. A., Li, L., Zhang, X., Daoud, R., Ryan, J., Ning, Z., Cheng, K., Nguyen, L. V., Abou-Samra, E. et al. (2018). Proteomic and metaproteomic approaches to understand host-microbe interactions. Analytical chemistry 90, 86-109.

Tang, W. H. and Hazen, S. L. (2017). The Gut Microbiome and Its Role in Cardiovascular Diseases. Circulation 135, 1008-1010.

Taubert, M., Vogt, C., Wubet, T., Kleinsteuber, S., Tarkka, M. T., Harms, H., Buscot, F., Richnow, H.-H., Von Bergen, M. and Seifert, J. (2012). Protein-SIP enables timeresolved analysis of the carbon flux in a sulfate-reducing, benzene-degrading microbial consortium. The ISME journal 6, 2291-2301.

Thompson, A., Schäfer, J., Kuhn, K., Kienle, S., Schwarz, J., Schmidt, G., Neumann, T. and Hamon, C. (2003). Tandem mass tags: a novel quantification strategy for comparative analysis of complex protein mixtures by MS/MS. Analytical chemistry 75, 1895-1904.

Tremlett, H., Bauer, K. C., Appel-Cresswell, S., Finlay, B. B. and Waubant, E. (2017). The gut microbiome in human neurological disease: A review. Ann Neurol 81, 369-382.

Verheggen, K., Ræder, H., Berven, F. S., Martens, L., Barsnes, H. and Vaudel, M. (2020). 
Anatomy and evolution of database search engines - a central component of mass spectrometry based proteomic workflows. Mass spectrometry reviews 39, 292-306.

Wang, Y., Ahn, T.-H., Li, Z. and Pan, C. (2013). Sipros/ProRata: a versatile informatics system for quantitative community proteomics. Bioinformatics 29, 2064-2065.

Webhofer, C., Zhang, Y., Brusis, J., Reckow, S., Landgraf, R., Maccarrone, G., Turck, C. W. and Filiou, M. D. (2013). 15N metabolic labeling: Evidence for a stable isotope effect on plasma protein levels and peptide chromatographic retention times. Journal of proteomics $88,27-33$.

Weisser, H. and Choudhary, J. S. (2017). Targeted feature detection for data-dependent shotgun proteomics. Journal of proteome research 16, 2964-2974.

Whipps, J., Lewis, K. and Cooke, R. (1988). Mycoparasitism and plant disease control. Fungi in biological control systems ., 161-187.

Wickham, H. (2016). ggplot2: elegant graphics for data analysis. Springer.

Xiao, L., Feng, Q., Liang, S., Sonne, S. B., Xia, Z., Qiu, X., Li, X., Long, H., Zhang, J., Zhang, D. et al. (2015). A catalog of the mouse gut metagenome. Nature biotechnology 33, 1103.

Young, W., Egert, M., Bassett, S. A. and Bibiloni, R. (2015). Detection of sialic acidutilising bacteria in a caecal community batch culture using RNA-based stable isotope probing. Nutrients 7, 2109-2124.

Zhang, X., Chen, W., Ning, Z., Mayne, J., Mack, D., Stintzi, A., Tian, R. and Figeys, D. 
(2017). Deep Metaproteomics Approach for the Study of Human Microbiomes. Anal Chem 89, 9407-9415.

Zhang, X., Deeke, S. A., Ning, Z., Starr, A. E., Butcher, J., Li, J., Mayne, J., Cheng, K., Liao, B., Li, L. et al. (2018a). Metaproteomics reveals associations between microbiome and intestinal extracellular vesicle proteins in pediatric inflammatory bowel disease. Nature communications 9, 1-14.

Zhang, X., Li, L., Mayne, J., Ning, Z., Stintzi, A. and Figeys, D. (2018b). Assessing the impact of protein extraction methods for human gut metaproteomics. J Proteomics 180, $120-127$. 
APPENDICES

A.1 Supplemental Figures 

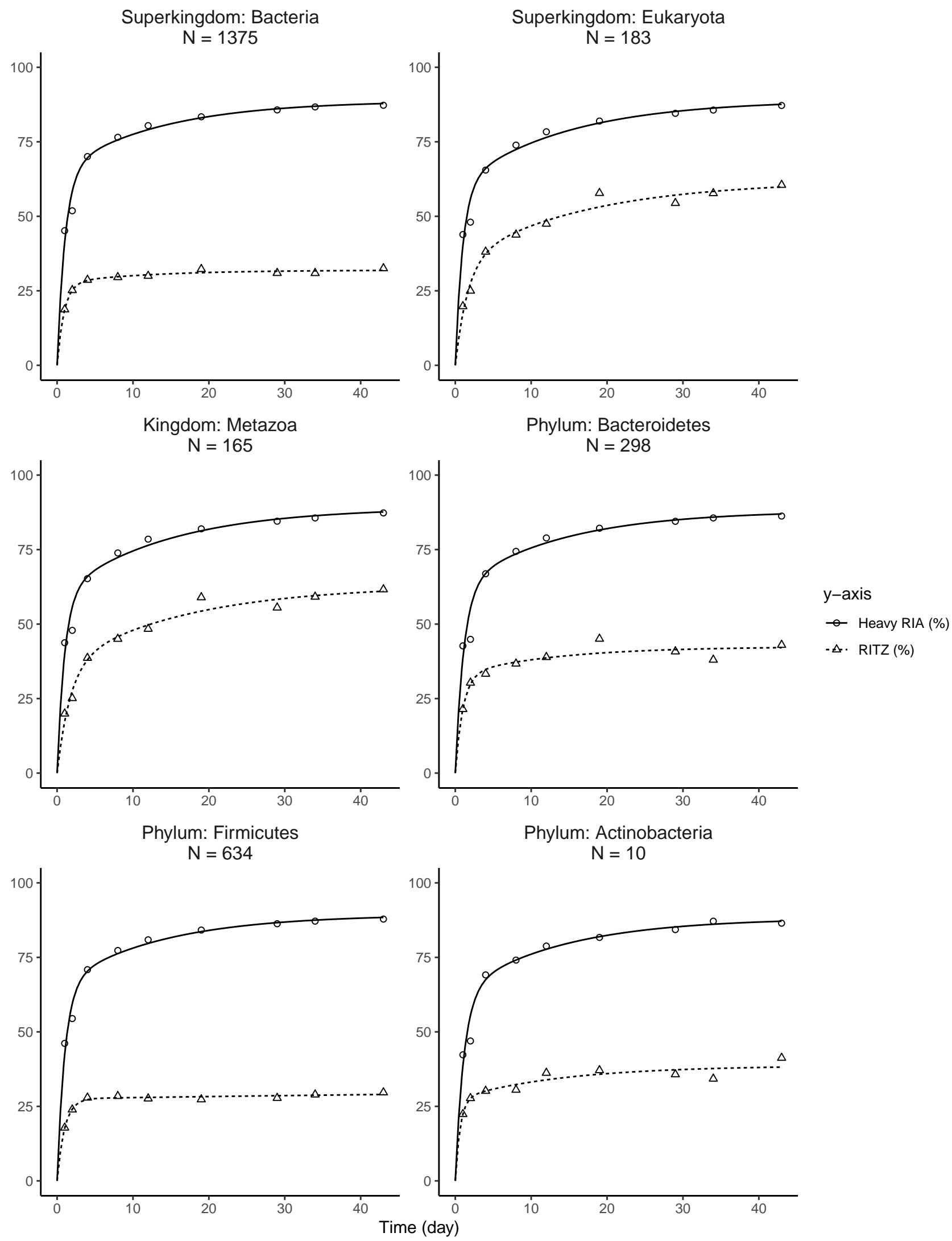
Phylum: Chordata

$\mathrm{N}=149$

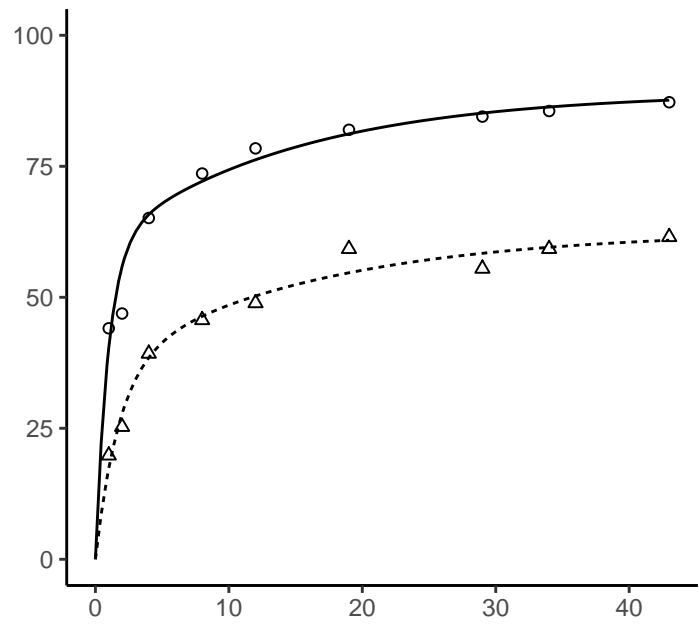

Phylum: Deferribacteres
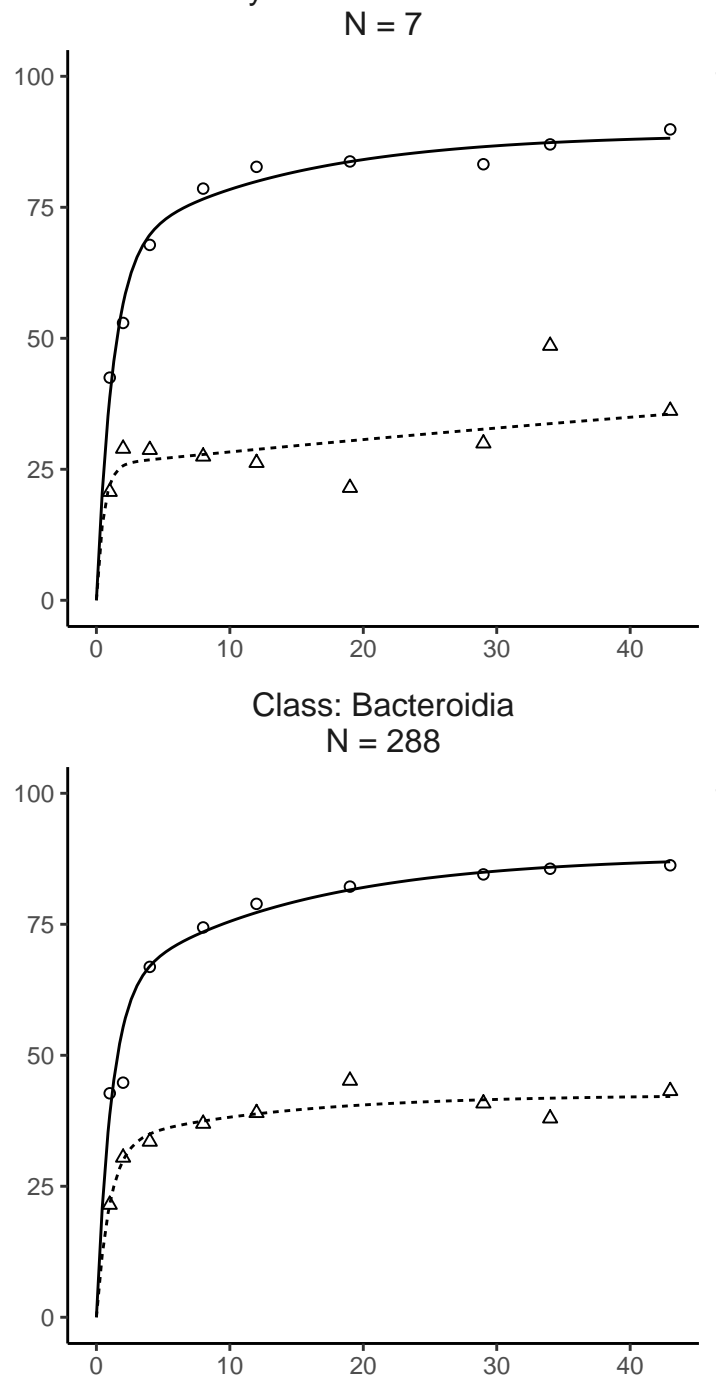

Phylum: Proteobacteria

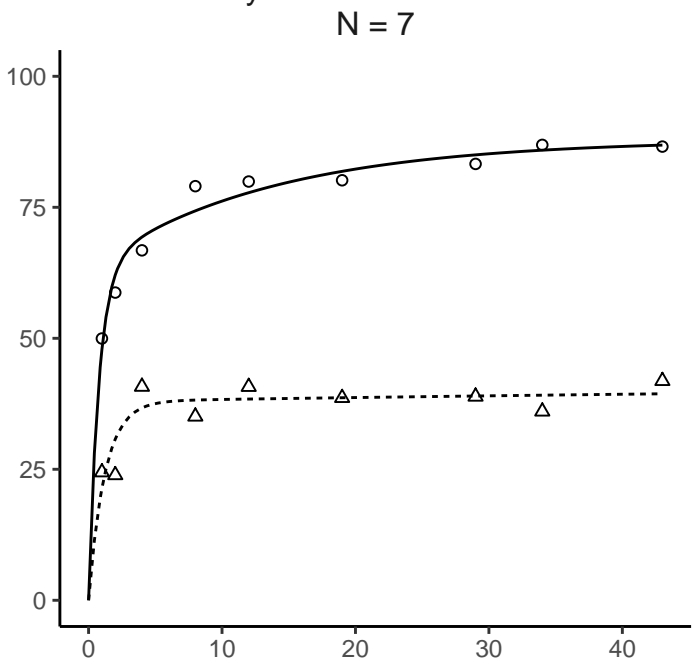

Phylum: Verrucomicrobia

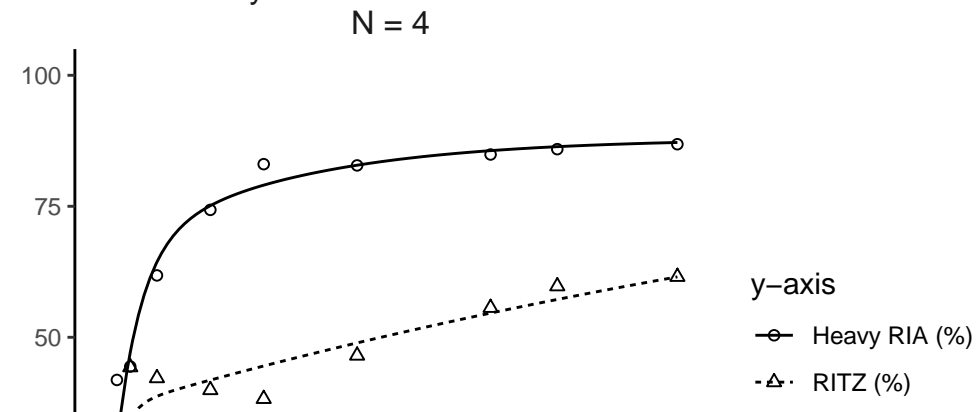

0

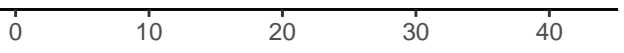

Class: Clostridia

$\mathrm{N}=338$

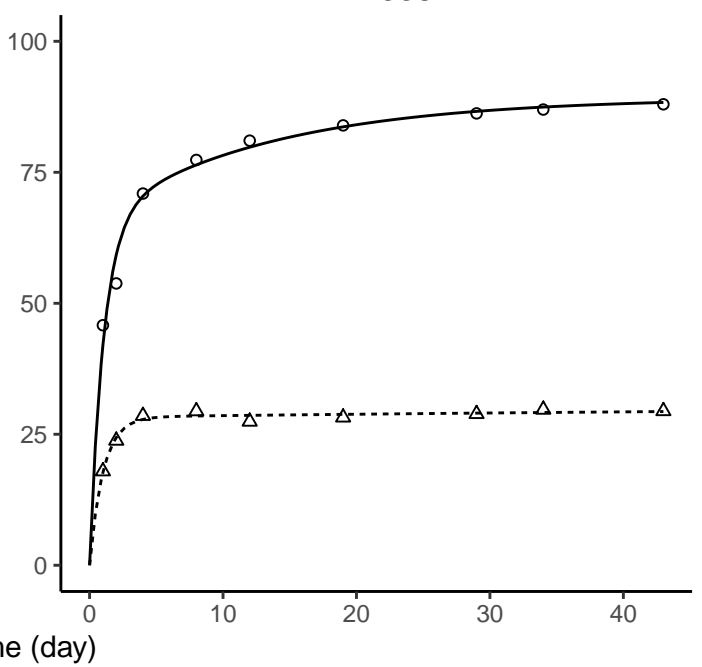



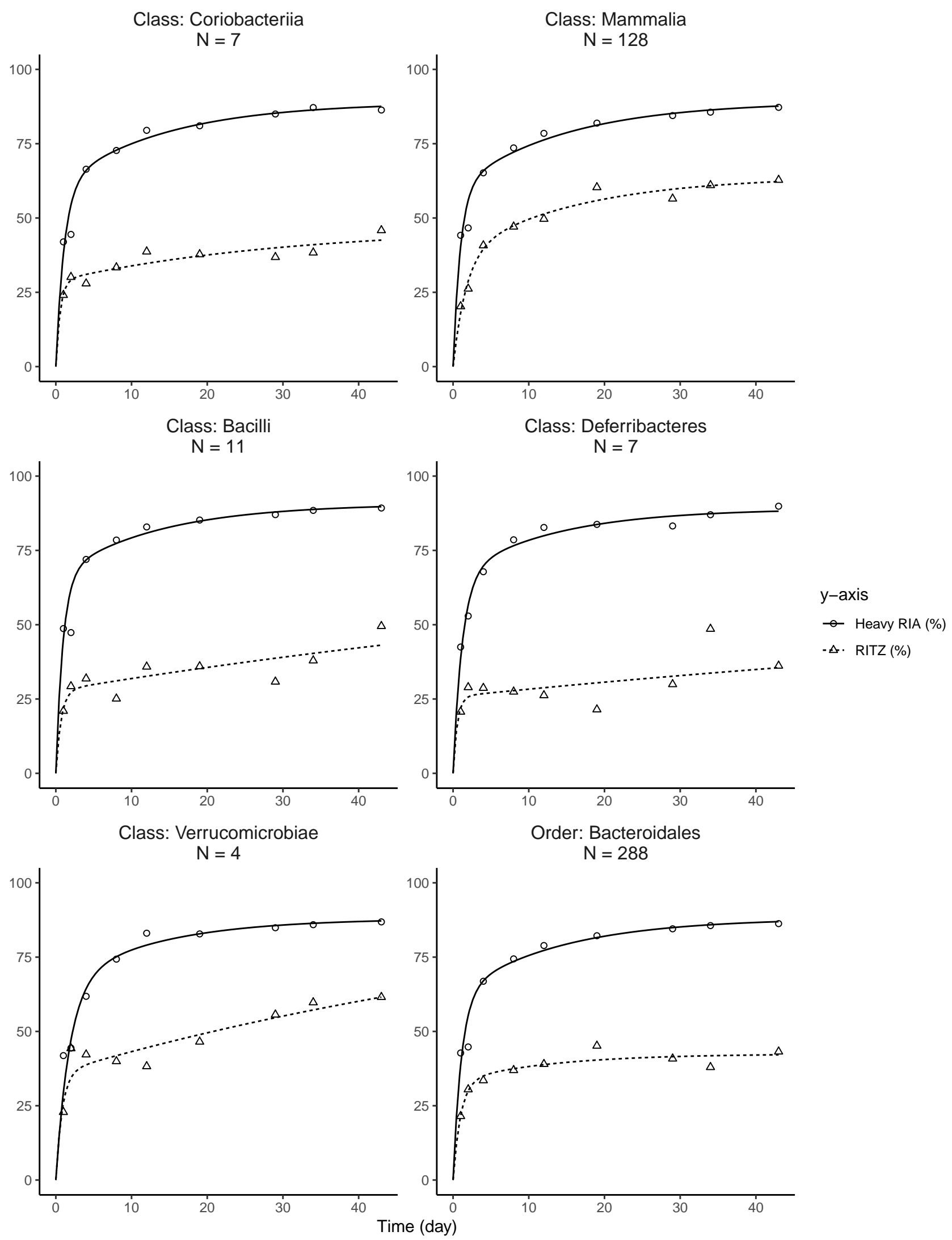

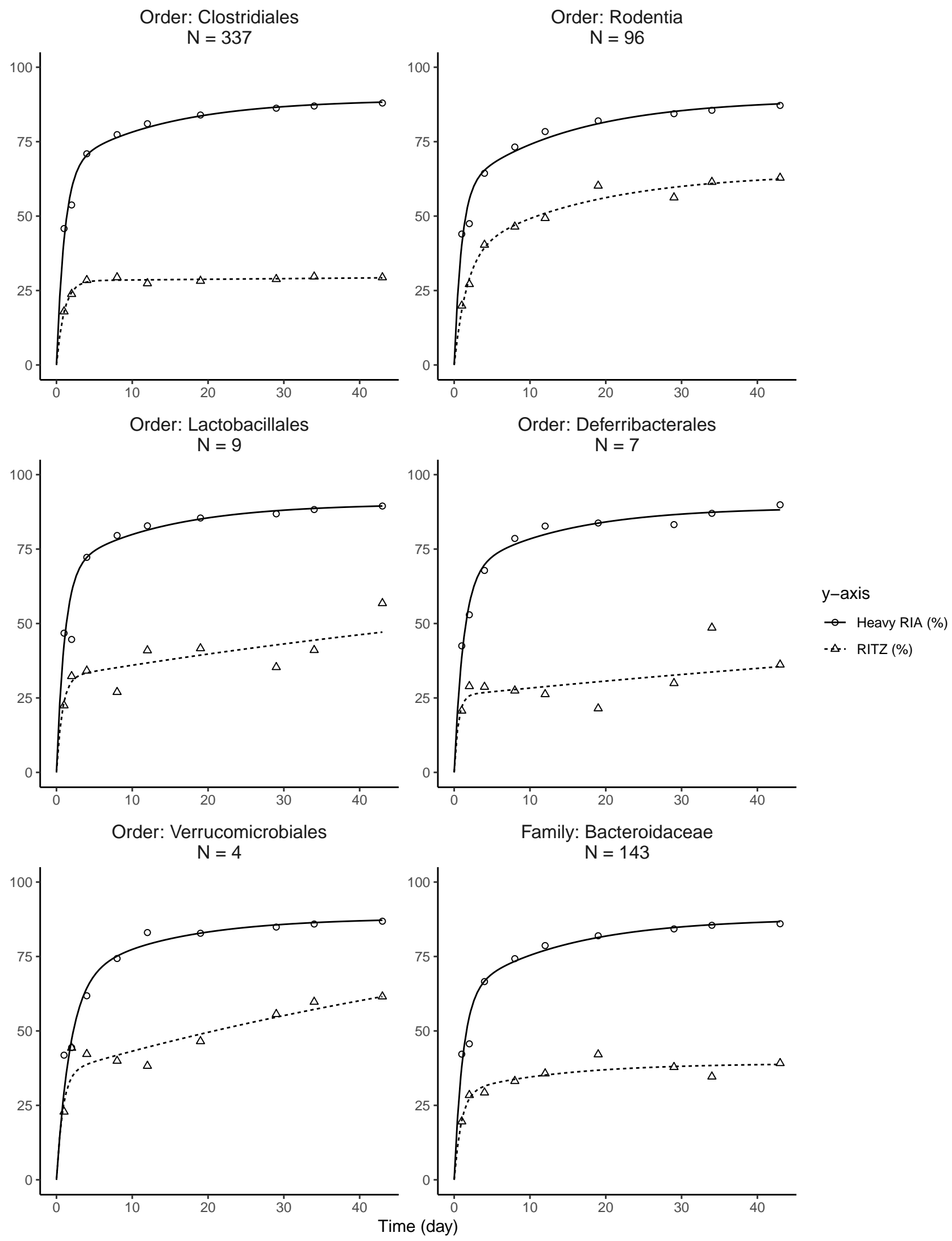
Family: Lachnospiraceae

$$
\mathrm{N}=90
$$

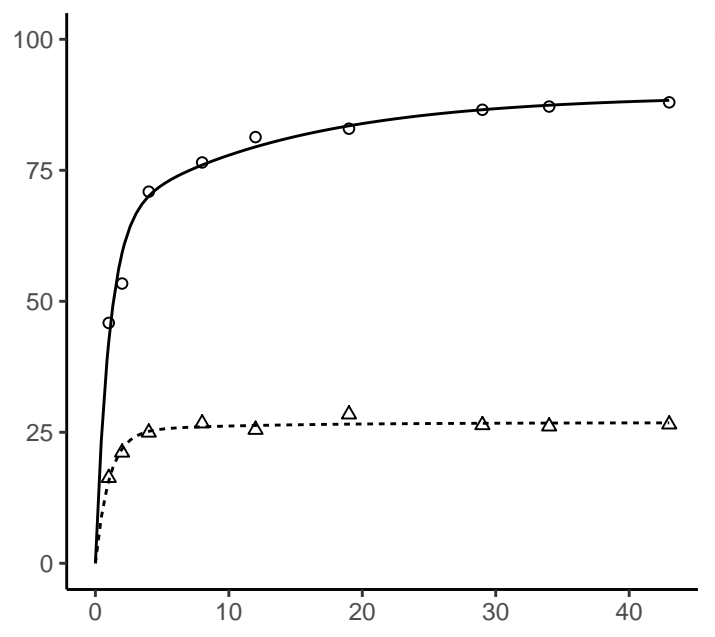

Family: Muridae

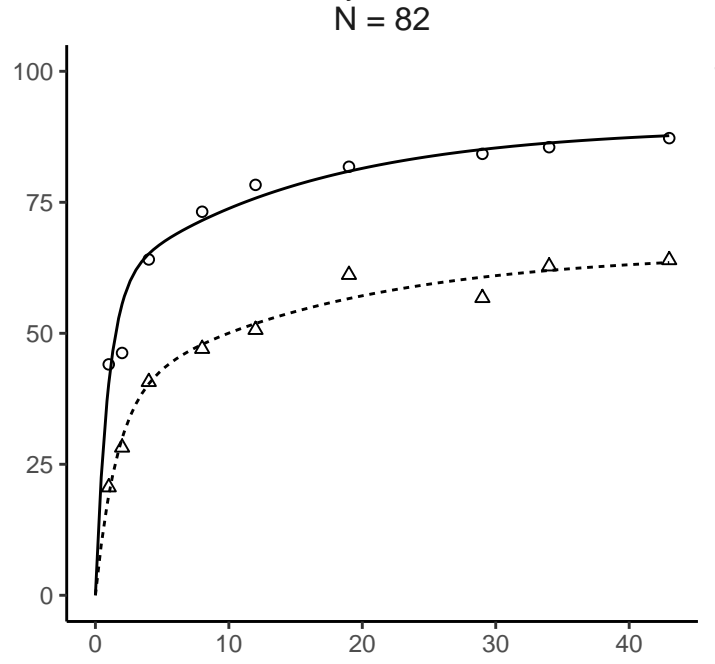

Family: Oscillospiraceae

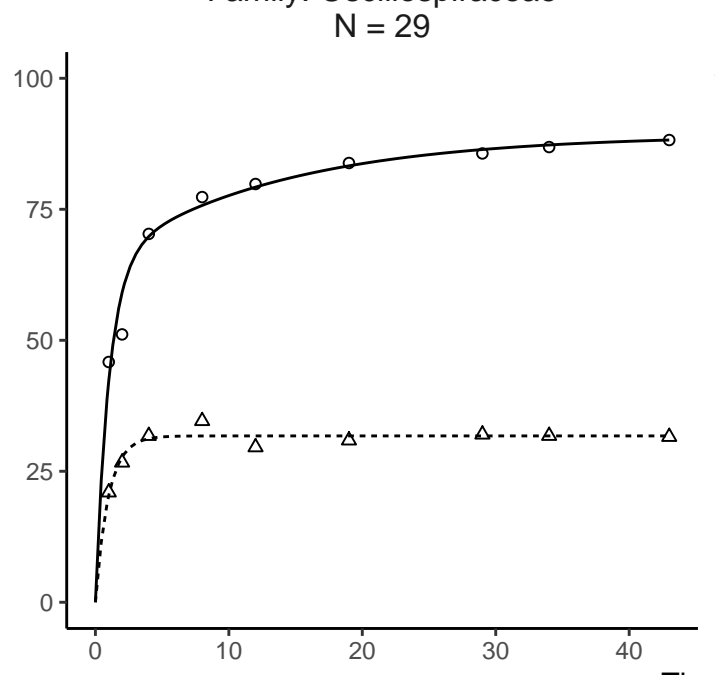

Family: Porphyromonadaceae

$\mathrm{N}=75$

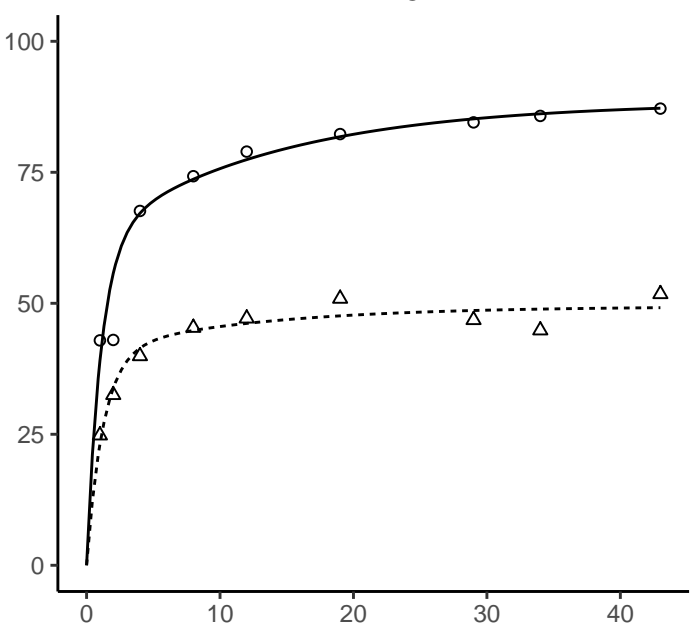

Family: Ruminococcaceae

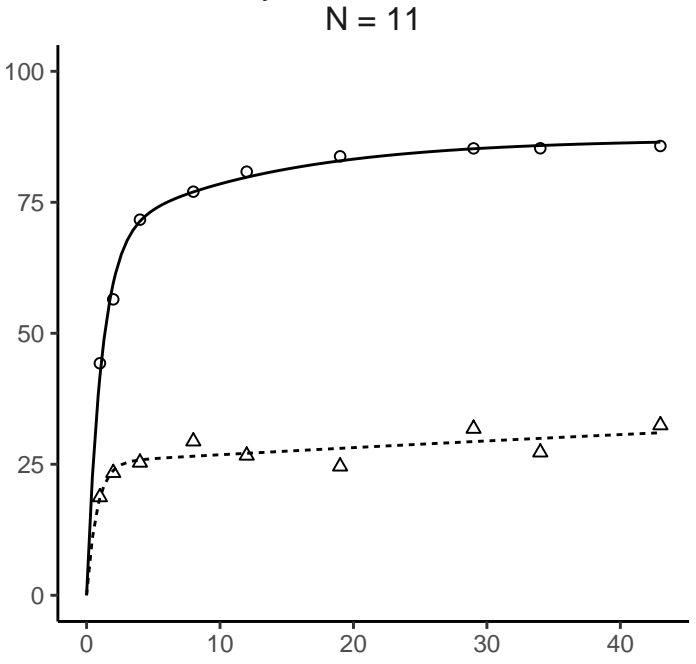

Family: Eubacteriaceae

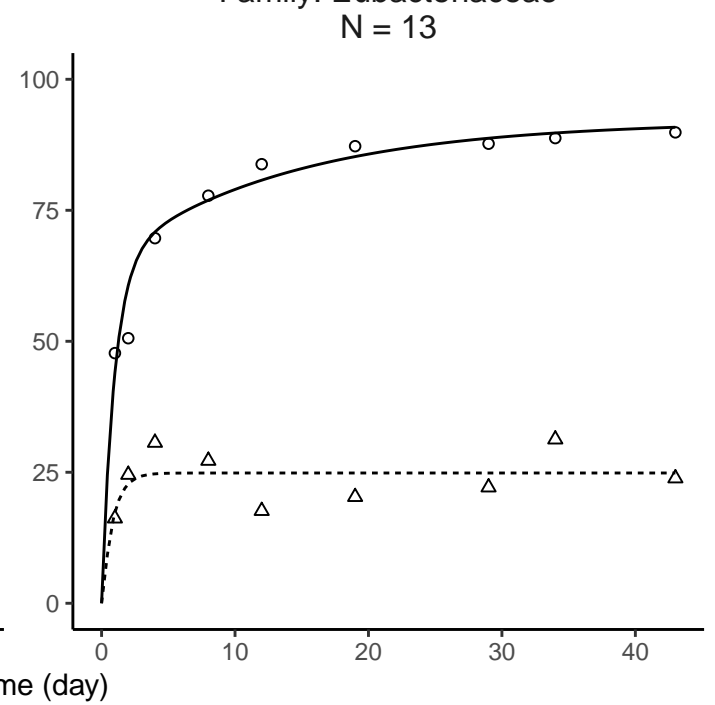

$y$-axis

- Heavy RIA (\%)

- $\Delta$. RITZ (\%) 
Family: Lactobacillaceae

$$
\mathrm{N}=8
$$

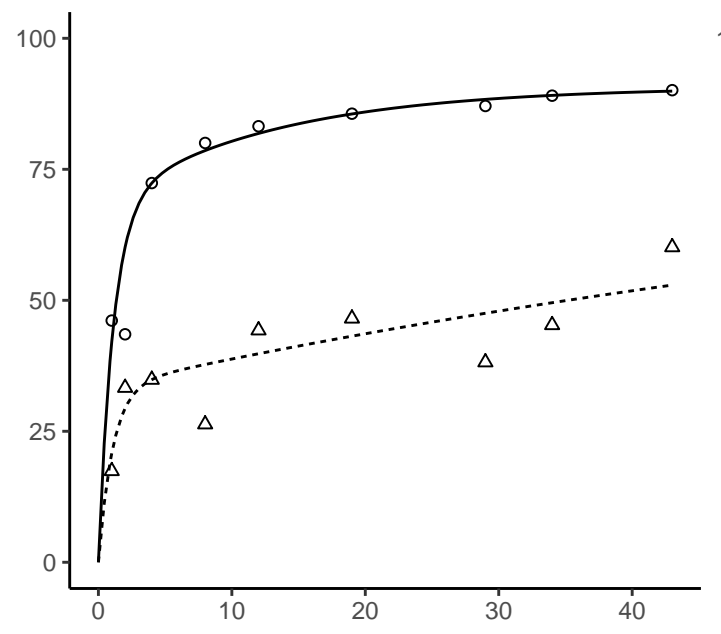

Family: Deferribacteraceae

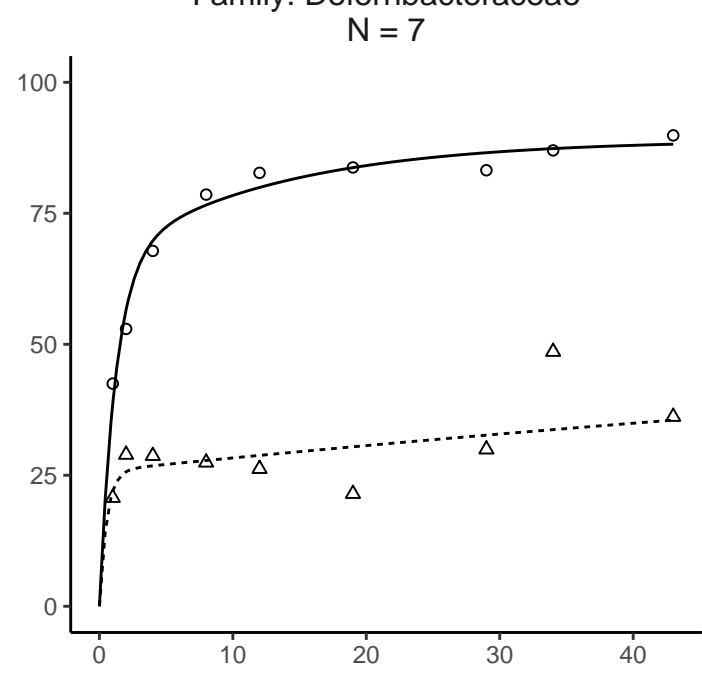

Genus: Bacteroides

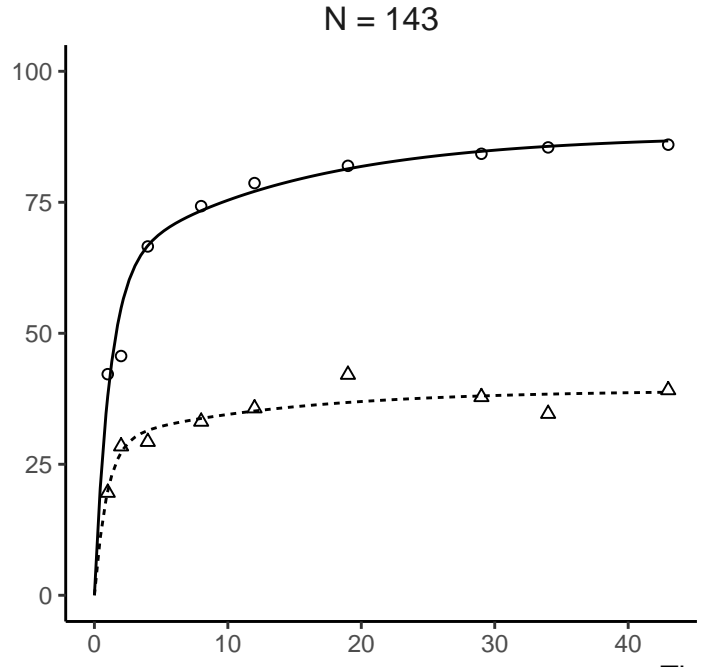

Family: Clostridiaceae

$\mathrm{N}=14$

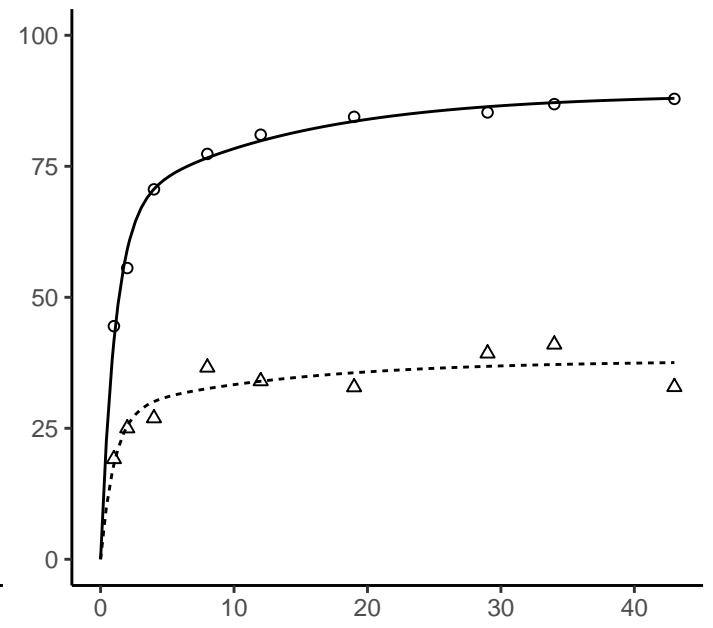

Family: Akkermansiaceae

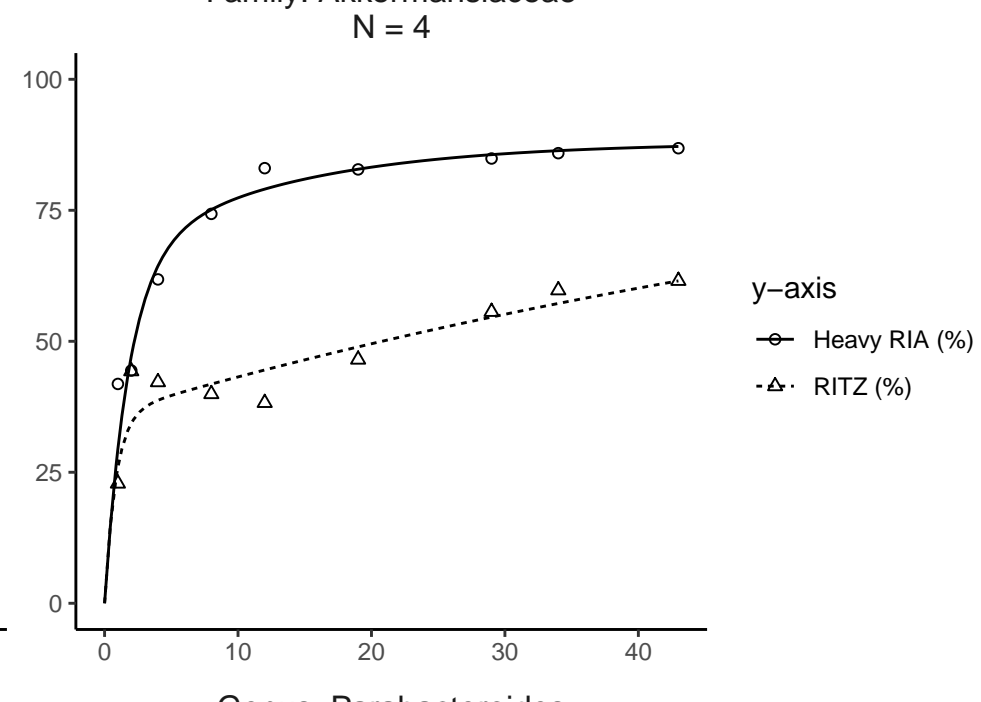



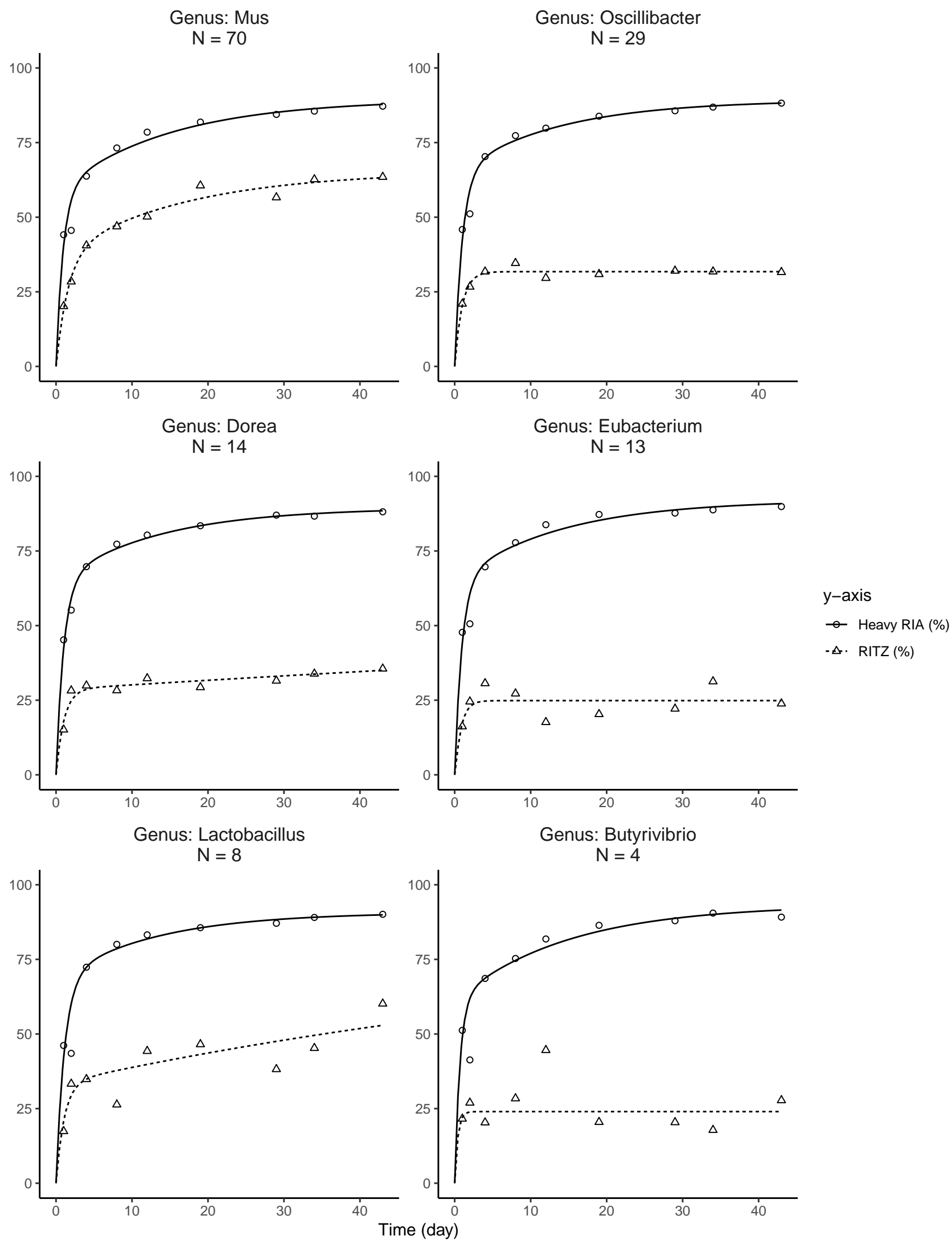

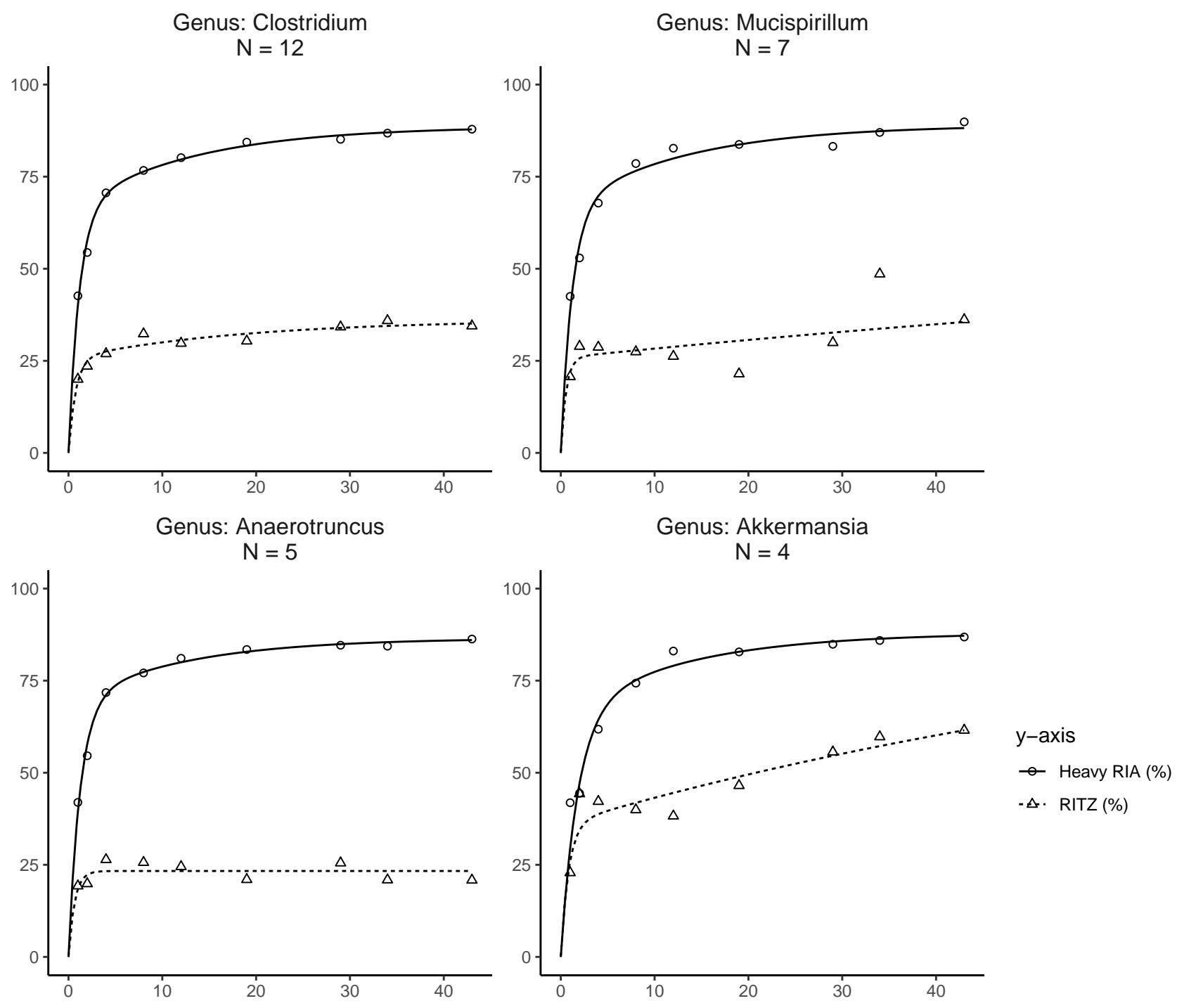

Species: Lachnospiraceae bacterium 28-4
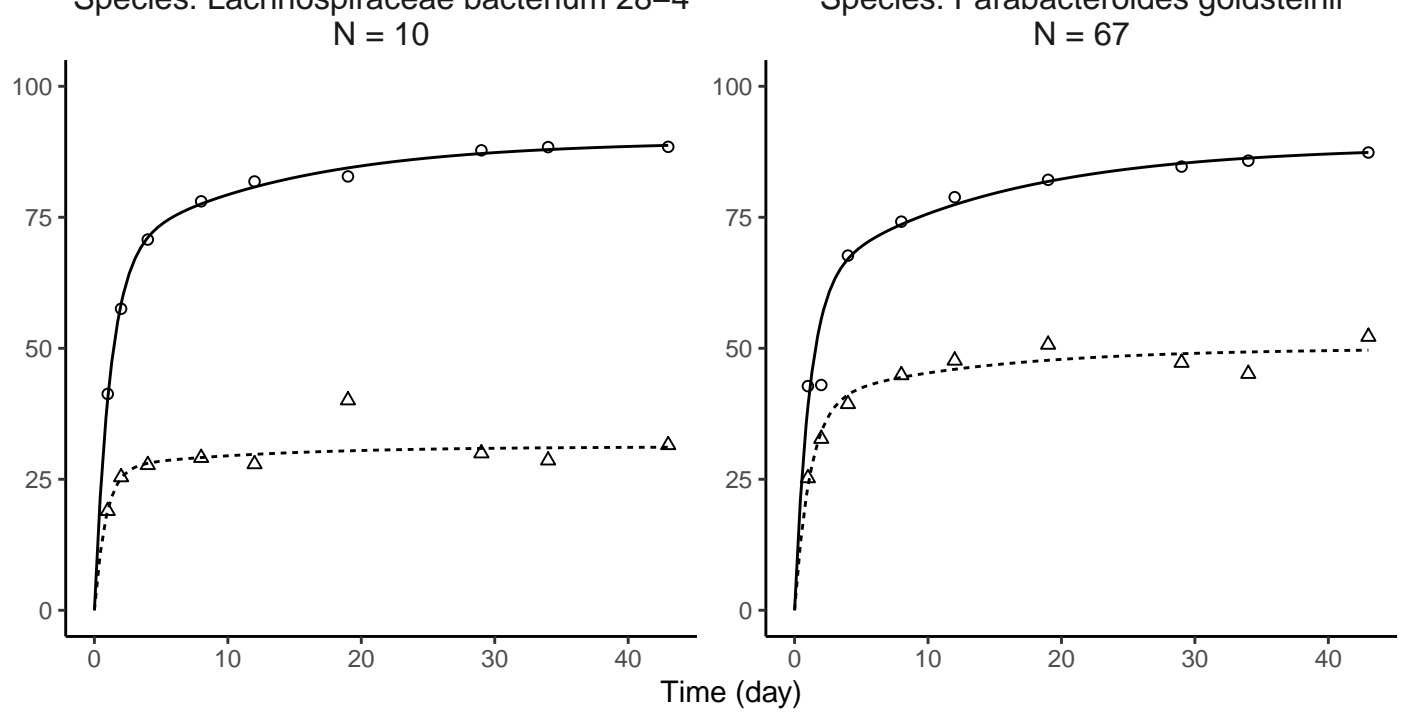

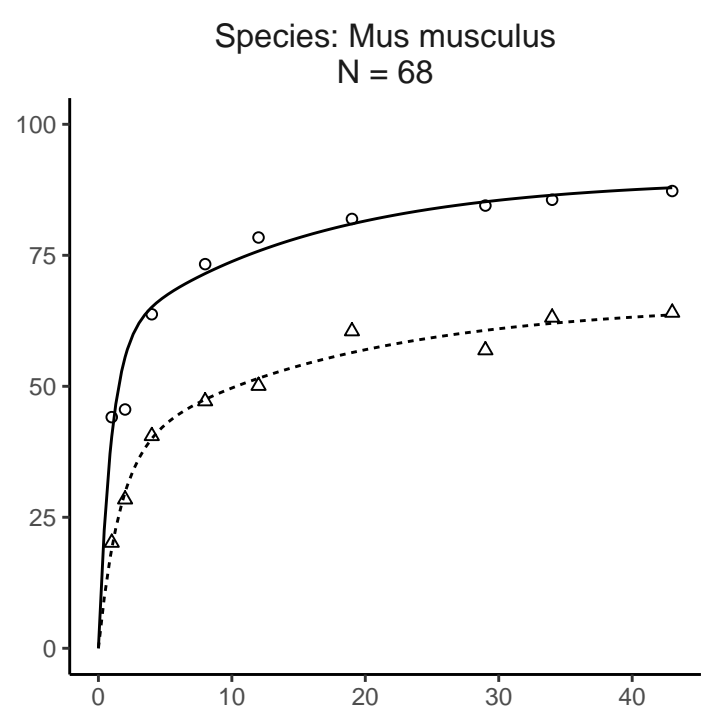

Species: Bacteroides vulgatus

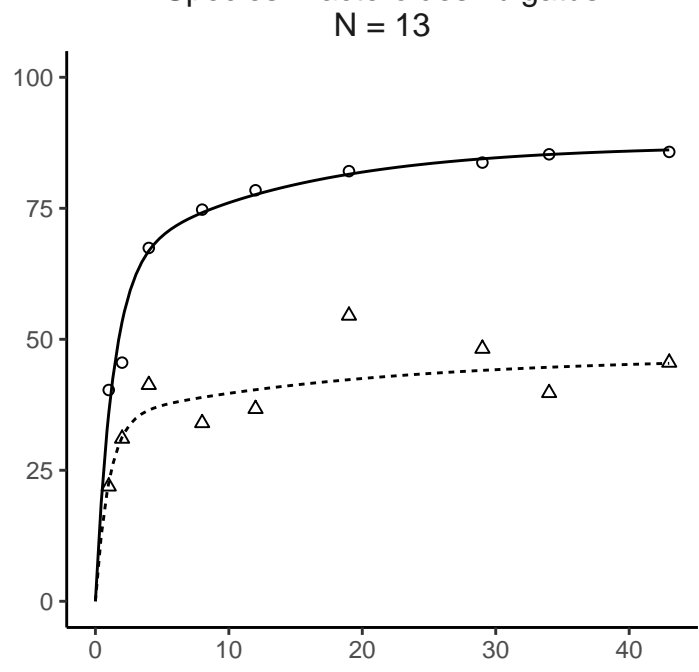

Species: Lachnospiraceae bacterium A4

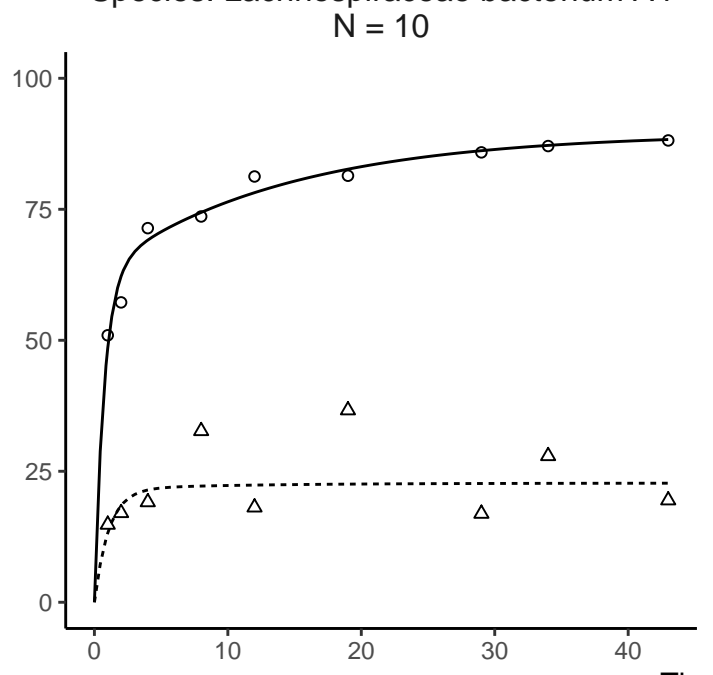

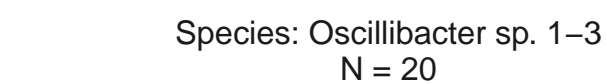
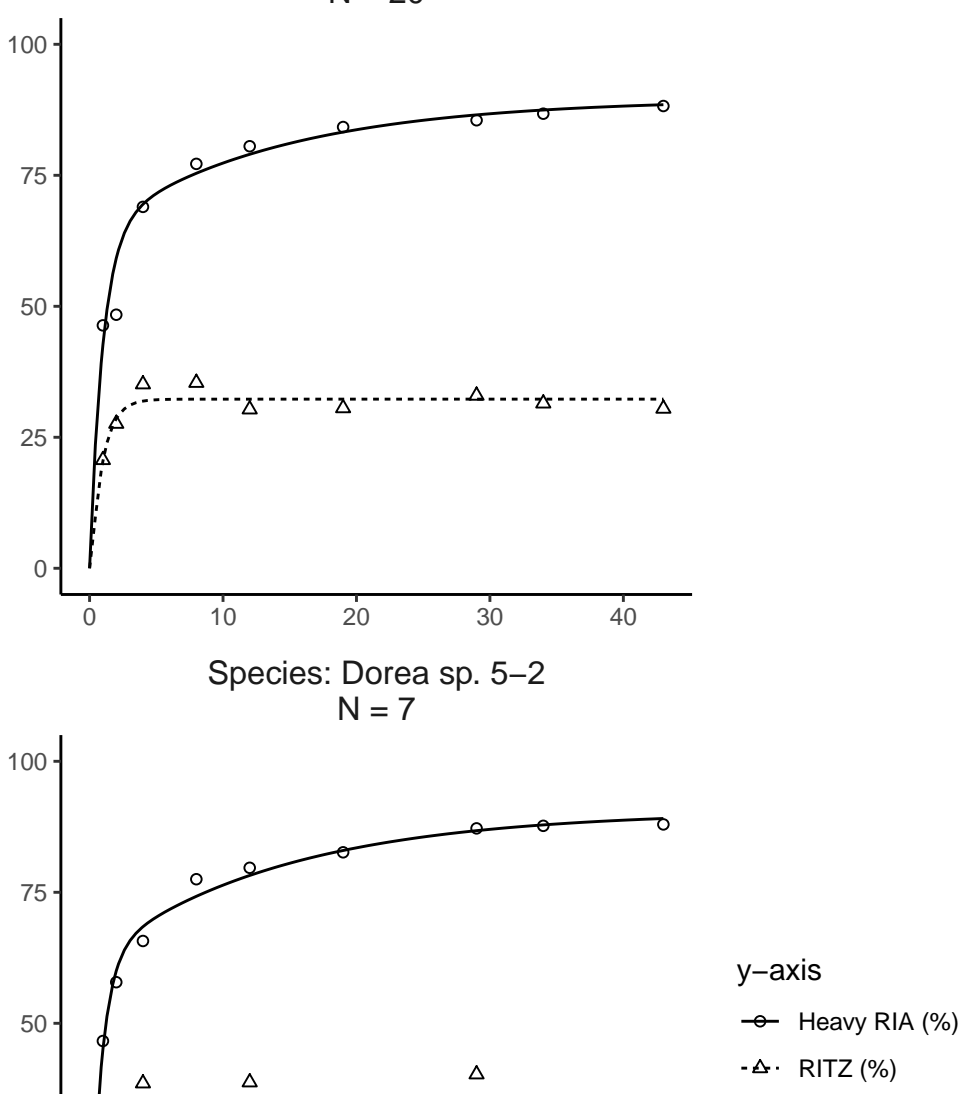

$$
0-
$$

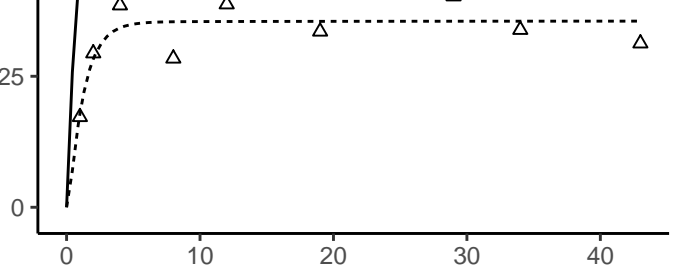

Species: Lachnospiraceae bacterium 10-1

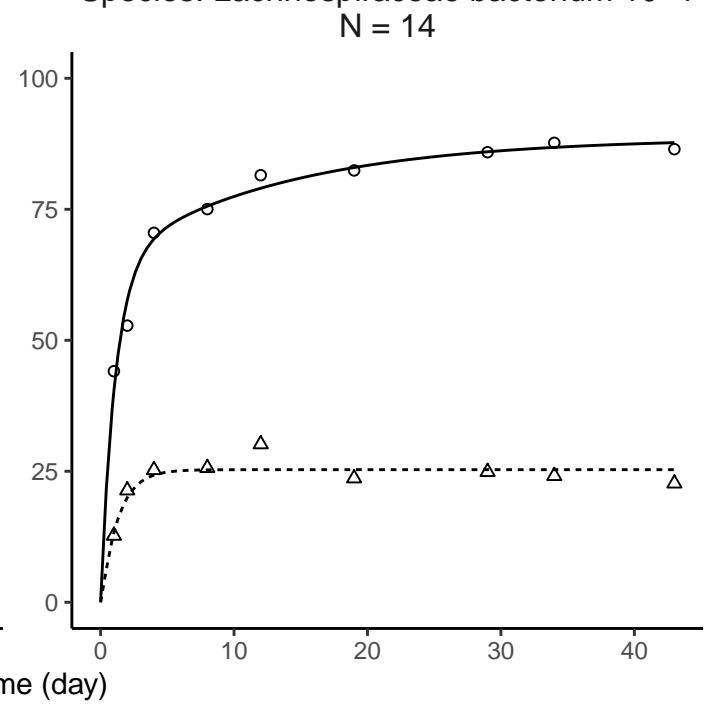




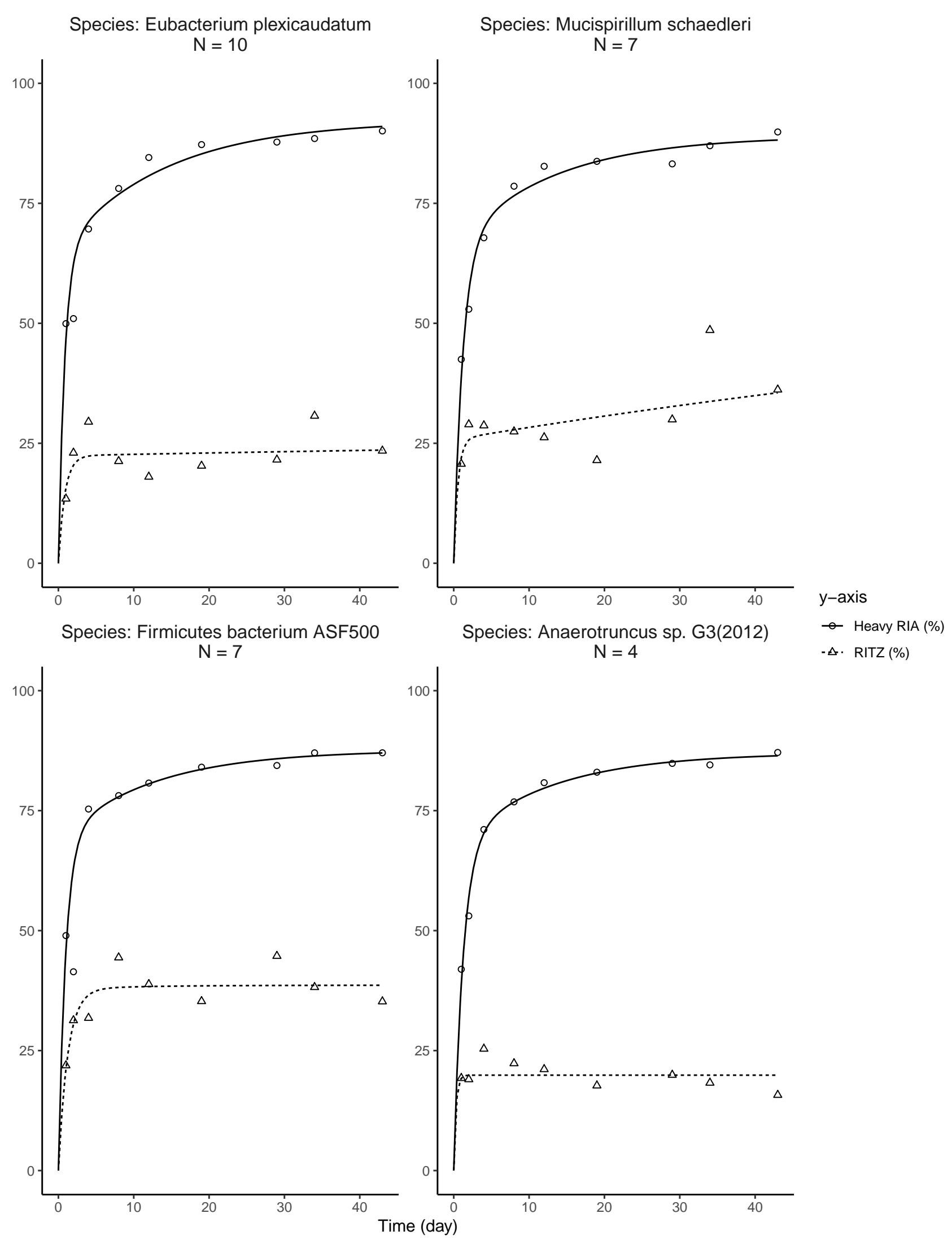


A.2 Supplemental Tables 
Table A.1: Average taxonomic RITZ from day 29 to 43

\begin{tabular}{|c|c|c|c|}
\hline Rank & $\mathrm{LCA}$ & RITZ mean (\%) & p.value \\
\hline Phylum & Firmicutes & 31.4 & 0 \\
\hline Phylum & Proteobacteria & 40.2 & $2.13 \times 10^{-18}$ \\
\hline Phylum & Bacteroidetes & 40.6 & 0 \\
\hline Phylum & Chordata & 60.2 & $5.38 \times 10^{-307}$ \\
\hline Phylum & Actinobacteria & 38.3 & $3.11 \times 10^{-17}$ \\
\hline Phylum & Euryarchaeota & 24.3 & 0.0183 \\
\hline Phylum & Verrucomicrobia & 58.2 & $5.01 \times 10^{-14}$ \\
\hline Phylum & Fusobacteria & 8.25 & 0.0379 \\
\hline Phylum & Deferribacteres & 27.4 & $4.17 \times 10^{-11}$ \\
\hline Phylum & Ascomycota & 27.7 & $6.96 \times 10^{-6}$ \\
\hline Phylum & Platyhelminthes & 30.6 & 0.0243 \\
\hline Phylum & Spirochaetes & 34.4 & 0.0407 \\
\hline Phylum & Cyanobacteria & 33.2 & 0.0167 \\
\hline Class & Clostridia & 31.9 & 0 \\
\hline Class & Bacteroidia & 40.6 & 0 \\
\hline Class & Mammalia & 62.7 & $1.87 \times 10^{-279}$ \\
\hline Class & Negativicutes & 10.3 & 0.00313 \\
\hline Class & Alphaproteobacteria & 41.9 & $1.43 \times 10^{-6}$ \\
\hline Class & Coriobacteriia & 40.4 & $2.75 \times 10^{-15}$ \\
\hline Class & Deltaproteobacteria & 60.5 & 0.00848 \\
\hline Class & Bacilli & 49.3 & $1.35 \times 10^{-19}$ \\
\hline Class & Thermococci & 24.3 & 0.0183 \\
\hline Class & Verrucomicrobiae & 58.2 & $5.01 \times 10^{-14}$ \\
\hline Class & Fusobacteriia & 8.25 & 0.0379 \\
\hline Class & Deferribacteres & 27.4 & $4.17 \times 10^{-11}$ \\
\hline Class & Actinobacteria & 33.6 & 0.000458 \\
\hline Class & Gammaproteobacteria & 33.9 & $7.68 \times 10^{-10}$ \\
\hline Class & Spirochaetia & 34.4 & 0.0407 \\
\hline Class & Flavobacteriia & 35.5 & 0.00621 \\
\hline Order & Clostridiales & 31.8 & 0 \\
\hline Order & Bacteroidales & 40.6 & 0 \\
\hline Order & Rodentia & 62.9 & $3.15 \times 10^{-228}$ \\
\hline Order & Selenomonadales & 10.3 & 0.00313 \\
\hline Order & Rhodobacterales & 34.8 & $1.01 \times 10^{-5}$ \\
\hline Order & Eggerthellales & 45.5 & $1.84 \times 10^{-7}$ \\
\hline Order & Lactobacillales & 53.5 & $2.3 \times 10^{-18}$ \\
\hline Order & Thermococcales & 24.3 & 0.0183 \\
\hline Order & Verrucomicrobiales & 58.2 & $5.01 \times 10^{-14}$ \\
\hline Order & Fusobacteriales & 8.25 & 0.0379 \\
\hline Order & Rhizobiales & 56.1 & 0.00621 \\
\hline Order & Deferribacterales & 27.4 & $4.17 \times 10^{-11}$ \\
\hline
\end{tabular}


Table A.1 - continued from previous page

\begin{tabular}{clll}
\hline Rank & & RITZ mean (\%) & \multicolumn{1}{c}{ p.value } \\
\hline Order & Desulfovibrionales & 88.2 & 0.00286 \\
Order & Xanthomonadales & 20.2 & 0.0355 \\
Order & Spirochaetales & 34.4 & 0.0407 \\
Order & Alteromonadales & 36.6 & 0.000228 \\
Order & Flavobacteriales & 35.5 & 0.00621 \\
Family & Lachnospiraceae & 30.7 & $3.73 \times 10^{-111}$ \\
Family & Bacteroidaceae & 38 & $1.47 \times 10^{-208}$ \\
Family & Rhodobacteraceae & 34.8 & $1.01 \times 10^{-5}$ \\
Family & Oscillospiraceae & 33 & $5.18 \times 10^{-35}$ \\
Family & Eggerthellaceae & 45.5 & $1.84 \times 10^{-7}$ \\
Family & Porphyromonadaceae & 46.7 & $1.44 \times 10^{-134}$ \\
Family & Muridae & 63.4 & $3.22 \times 10^{-202}$ \\
Family & Clostridiaceae & 42.6 & $2.49 \times 10^{-26}$ \\
Family & Lactobacillaceae & 54 & $6.03 \times 10^{-17}$ \\
Family & Thermococcaceae & 24.3 & 0.0183 \\
Family & Ruminococcaceae & 34 & $7.62 \times 10^{-15}$ \\
Family & Eubacteriaceae & 30 & $1.54 \times 10^{-12}$ \\
Family & Akkermansiaceae & 58.2 & $5.01 \times 10^{-14}$ \\
Family & Fusobacteriaceae & 8.25 & 0.0379 \\
Family & Bradyrhizobiaceae & 56.1 & 0.00621 \\
Family & Deferribacteraceae & 27.4 & $4.17 \times 10^{-11}$ \\
Family & Desulfovibrionaceae & 88.2 & 0.00286 \\
Family & Xanthomonadaceae & 20.2 & 0.0355 \\
Family & Spirochaetaceae & 34.4 & 0.0407 \\
Family & Flavobacteriaceae & 36.9 & 0.00589 \\
Genus & Bacteroides & 38 & $1.47 \times 10^{-208}$ \\
Genus & Oscillibacter & 33 & $5.18 \times 10^{-35}$ \\
Genus & Blautia & 44.5 & 0.00113 \\
Genus & Parabacteroides & 46.9 & $7.02 \times 10^{-133}$ \\
Genus & Clostridium & 40.9 & $3.48 \times 10^{-20}$ \\
Genus & Mus & 63.5 & $4.16 \times 10^{-171}$ \\
Genus & Lactobacillus & 53.5 & $1.21 \times 10^{-15}$ \\
Genus & Ruminiclostridium & 32.2 & 0.000126 \\
Genus & Roseburia & 36.6 & 0.000209 \\
Genus & Eubacterium & 29.4 & $3.57 \times 10^{-12}$ \\
Genus & Akkermansia & 58.2 & $5.01 \times 10^{-14}$ \\
Genus & Bradyrhizobium & 56.1 & 0.00621 \\
Genus & Herbinix & 23.8 & 0.0431 \\
Genus & Mucispirillum & 27.4 & $4.17 \times 10^{-11}$ \\
Genus & Lachnoclostridium & 19.9 & 0.000259 \\
Genus & Dorea & $0.24 \times 10^{-17}$ \\
Genus & Coprococcus & & 0.0306 \\
\hline
\end{tabular}

Continued on next page 
Table A.1 - continued from previous page

\begin{tabular}{clll}
\hline Rank & \multicolumn{1}{c}{ LCA } & RITZ mean (\%) & \multicolumn{1}{c}{ p.value } \\
\hline Genus & Anaerotruncus & 24.4 & $5.11 \times 10^{-7}$ \\
Genus & Butyrivibrio & 22.8 & 0.000168 \\
Genus & Flavonifractor & 37.3 & 0.00045 \\
Genus & Subdoligranulum & 41 & 0.00662 \\
Genus & Butyricicoccus & 71.8 & 0.00662 \\
Genus & Ruminococcus & 51.2 & 0.00013 \\
Genus & Enterorhabdus & 34.4 & 0.0493 \\
Genus & Treponema & 27.4 & 0.0407 \\
Species & Lachnospiraceae bacterium 10-1 & 31.1 & $3.62 \times 10^{-12}$ \\
Species & Lachnospiraceae bacterium A4 & $310^{-17}$ \\
Species & Lachnospiraceae bacterium 28-4 & 35.2 & $7.62 \times 10^{-15}$ \\
Species & Lachnospiraceae bacterium COE1 & 38.9 & 0.000236 \\
Species & Parabacteroides goldsteinii & 47.3 & $7.54 \times 10^{-120}$ \\
Species & Clostridium sp. ASF502 & 55.2 & $1.3 \times 10^{-12}$ \\
Species & Mus musculus & 64 & $7.41 \times 10^{-166}$ \\
Species & Lachnospiraceae bacterium 3-2 & 34.9 & 0.000187 \\
Species & Lachnospiraceae bacterium JC7 & 21 & 0.000461 \\
Species & Ruminiclostridium sp. KB18 & 28.6 & 0.00116 \\
Species & Eubacterium plexicaudatum & 29.2 & $1.08 \times 10^{-8}$ \\
Species & Oscillibacter sp. 1-3 & 34.7 & $8.04 \times 10^{-30}$ \\
Species & Akkermansia muciniphila & 57 & $2.46 \times 10^{-8}$ \\
Species & Lachnospiraceae bacterium M18-1 & 45 & $2.31 \times 10^{-7}$ \\
Species & Mucispirillum schaedleri & 27.4 & $4.17 \times 10^{-11}$ \\
Species & Dorea sp. 5-2 & 35.5 & $1.84 \times 10^{-9}$ \\
Species & Bacteroides vulgatus & 48.9 & $2.98 \times 10^{-26}$ \\
Species & Lachnospiraceae bacterium A2 & 41.5 & $9.46 \times 10^{-5}$ \\
Species & Firmicutes bacterium ASF500 & 43.3 & $2.98 \times 10^{-14}$ \\
Species & Anaerotruncus sp. G3(2012) & 21.9 & $9.51 \times 10^{-6}$ \\
Species & Flavonifractor plautii & 40.3 & 0.000455 \\
Species & Subdoligranulum variabile & 41 & 0.00662 \\
Species & Oscillibacter sp. KLE 1745 & 59.3 & 0.0209 \\
Species & Enterorhabdus caecimuris & 51.2 & 0.0493 \\
Species & Oscillibacter valericigenes & 29.1 & 0.00267 \\
Species & Butyrivibrio crossotus & 17.3 & 0.00128 \\
Species & Eubacterium sp. 14-2 & 32.3 & 0.000461 \\
Species & Coprococcus comes & 35 & 0.0306 \\
\hline & & & \\
& &
\end{tabular}


Table A.2: Average taxonomic RITZ from day 29 to 43

\begin{tabular}{|c|c|c|c|c|}
\hline Rank & LCA & NOG category & RITZ mean (\%) & p-value \\
\hline Phylum & Firmicutes & Lipid transport and metabolism & 28.4 & $8.23 \times 10^{-50}$ \\
\hline Phylum & Proteobacteria & Carbohydrate transport and metabolism & 35.7 & $6.85 \times 10^{-7}$ \\
\hline Phylum & Bacteroidetes & Carbohydrate transport and metabolism & 35 & $2.95 \times 10^{-6}$ \\
\hline Phylum & Chordata & Intracellular trafficking, secretion, and vesicular transport & 62.3 & $6.72 \times 10^{-37}$ \\
\hline Phylum & Firmicutes & Energy production and conversion & 27 & $2.04 \times 10^{-156}$ \\
\hline Phylum & Firmicutes & Carbohydrate transport and metabolism & 33.5 & $7.33 \times 10^{-222}$ \\
\hline Phylum & Bacteroidetes & Cell wall/membrane/envelope biogenesis & 42.6 & $1.54 \times 10^{-51}$ \\
\hline Phylum & Firmicutes & Cell motility & 32.7 & $3.04 \times 10^{-45}$ \\
\hline Phylum & Proteobacteria & Amino acid transport and metabolism & 42.8 & $3.26 \times 10^{-5}$ \\
\hline Phylum & Firmicutes & Amino acid transport and metabolism & 36.2 & $8.73 \times 10^{-83}$ \\
\hline Phylum & Firmicutes & Translation, ribosomal structure and biogenesis & 31.1 & $1.07 \times 10^{-107}$ \\
\hline Phylum & Bacteroidetes & Inorganic ion transport and metabolism & 42.9 & $3.45 \times 10^{-84}$ \\
\hline Phylum & Firmicutes & Posttranslational modification, protein turnover, chaperones & 27.6 & $3 \times 10^{-34}$ \\
\hline Phylum & Actinobacteria & Amino acid transport and metabolism & 41.6 & $1.16 \times 10^{-11}$ \\
\hline Phylum & Firmicutes & Secondary metabolites biosynthesis, transport and catabolism & 35.3 & 0.0155 \\
\hline Phylum & Chordata & Inorganic ion transport and metabolism & 62.3 & $9.16 \times 10^{-36}$ \\
\hline Phylum & Firmicutes & Nucleotide transport and metabolism & 43 & $6.45 \times 10^{-22}$ \\
\hline Phylum & Chordata & Amino acid transport and metabolism & 56.5 & $1.38 \times 10^{-69}$ \\
\hline Phylum & Chordata & Posttranslational modification, protein turnover, chaperones & 56 & $4.63 \times 10^{-89}$ \\
\hline Phylum & Firmicutes & Cell wall/membrane/envelope biogenesis & 29.2 & 0.0386 \\
\hline Phylum & Chordata & Signal transduction mechanisms & 65.9 & $9.49 \times 10^{-44}$ \\
\hline Phylum & Firmicutes & Replication, recombination and repair & 31.6 & 0.00661 \\
\hline Phylum & Bacteroidetes & Energy production and conversion & 37.2 & $8.75 \times 10^{-19}$ \\
\hline Phylum & Verrucomicrobia & Translation, ribosomal structure and biogenesis & 67.2 & $3.14 \times 10^{-6}$ \\
\hline Phylum & Fusobacteria & Lipid transport and metabolism & 8.25 & 0.0355 \\
\hline Phylum & Verrucomicrobia & Carbohydrate transport and metabolism & 59.1 & $6.67 \times 10^{-6}$ \\
\hline Phylum & Chordata & Cytoskeleton & 41.6 & $3.46 \times 10^{-5}$ \\
\hline Phylum & Bacteroidetes & Intracellular trafficking, secretion, and vesicular transport & 37.8 & $3.61 \times 10^{-23}$ \\
\hline Phylum & Chordata & Carbohydrate transport and metabolism & 53.8 & $1.92 \times 10^{-25}$ \\
\hline Phylum & Bacteroidetes & Lipid transport and metabolism & 28.3 & 0.0466 \\
\hline Phylum & Bacteroidetes & Posttranslational modification, protein turnover, chaperones & 37.5 & 0.027 \\
\hline Phylum & Bacteroidetes & Amino acid transport and metabolism & 50.6 & $1.71 \times 10^{-8}$ \\
\hline Phylum & Actinobacteria & Energy production and conversion & 35.7 & $4.37 \times 10^{-6}$ \\
\hline Phylum & Chordata & Defense mechanisms & 87.3 & $1.56 \times 10^{-20}$ \\
\hline Phylum & Bacteroidetes & Translation, ribosomal structure and biogenesis & 35.5 & $1.52 \times 10^{-6}$ \\
\hline Phylum & Firmicutes & Transcription & 36 & 0.000898 \\
\hline Phylum & Proteobacteria & Energy production and conversion & 52.6 & 0.000205 \\
\hline Phylum & Firmicutes & Inorganic ion transport and metabolism & 22.4 & $1.24 \times 10^{-7}$ \\
\hline Phylum & Deferribacteres & Energy production and conversion & 31.9 & $1.29 \times 10^{-6}$ \\
\hline Phylum & Chordata & Extracellular structures & 61.5 & $1.33 \times 10^{-16}$ \\
\hline Phylum & Chordata & Secondary metabolites biosynthesis, transport and catabolism & 50.9 & 0.00664 \\
\hline Phylum & Chordata & Energy production and conversion & 51.3 & $1.63 \times 10^{-10}$ \\
\hline Phylum & Actinobacteria & Translation, ribosomal structure and biogenesis & 20 & 0.00066 \\
\hline Phylum & Ascomycota & Carbohydrate transport and metabolism & 27.3 & 0.000707 \\
\hline Phylum & Firmicutes & Intracellular trafficking, secretion, and vesicular transport & 47 & 0.00333 \\
\hline Phylum & Proteobacteria & Lipid transport and metabolism & 30.7 & 0.00367 \\
\hline Class & Clostridia & Lipid transport and metabolism & 26.3 & $1.41 \times 10^{-22}$ \\
\hline Class & Bacteroidia & Carbohydrate transport and metabolism & 37.3 & $7.4 \times 10^{-6}$ \\
\hline Class & Mammalia & Intracellular trafficking, secretion, and vesicular transport & 69.3 & $1.42 \times 10^{-28}$ \\
\hline Class & Clostridia & Energy production and conversion & 26.9 & $2.12 \times 10^{-94}$ \\
\hline Class & Clostridia & Carbohydrate transport and metabolism & 35.1 & $1.6 \times 10^{-144}$ \\
\hline Class & Bacteroidia & Cell wall/membrane/envelope biogenesis & 43.3 & $3.59 \times 10^{-48}$ \\
\hline Class & Clostridia & Cell motility & 34 & $4.94 \times 10^{-34}$ \\
\hline Class & Alphaproteobacteria & Amino acid transport and metabolism & 37.2 & 0.00125 \\
\hline Class & Clostridia & Translation, ribosomal structure and biogenesis & 35.2 & $9.49 \times 10^{-44}$ \\
\hline Class & Bacteroidia & Inorganic ion transport and metabolism & 42.4 & $1.21 \times 10^{-73}$ \\
\hline Class & Clostridia & Posttranslational modification, protein turnover, chaperones & 26.2 & $1.55 \times 10^{-22}$ \\
\hline Class & Coriobacteriia & Amino acid transport and metabolism & 45.2 & $2.38 \times 10^{-12}$ \\
\hline Class & Clostridia & Amino acid transport and metabolism & 35.4 & $1.6 \times 10^{-30}$ \\
\hline Class & Clostridia & Secondary metabolites biosynthesis, transport and catabolism & 35.3 & 0.0155 \\
\hline Class & Mammalia & Inorganic ion transport and metabolism & 64.5 & $5.16 \times 10^{-34}$ \\
\hline Class & Clostridia & Nucleotide transport and metabolism & 42.9 & $8.8 \times 10^{-9}$ \\
\hline Class & Mammalia & Amino acid transport and metabolism & 56.6 & $1.96 \times 10^{-58}$ \\
\hline Class & Mammalia & Posttranslational modification, protein turnover, chaperones & 57.6 & $5.69 \times 10^{-81}$ \\
\hline Class & Bacilli & Translation, ribosomal structure and biogenesis & 49.5 & $2.4 \times 10^{-5}$ \\
\hline Class & Mammalia & Signal transduction mechanisms & 65.9 & $9.49 \times 10^{-44}$ \\
\hline Class & Bacteroidia & Energy production and conversion & 37.3 & $8.85 \times 10^{-19}$ \\
\hline Class & Verrucomicrobiae & Translation, ribosomal structure and biogenesis & 67.2 & $3.14 \times 10^{-6}$ \\
\hline Class & Fusobacteriia & Lipid transport and metabolism & 8.25 & 0.0355 \\
\hline Class & Verrucomicrobiae & Carbohydrate transport and metabolism & 59.1 & $6.67 \times 10^{-6}$ \\
\hline Class & Mammalia & Cytoskeleton & 41.6 & $3.46 \times 10^{-5}$ \\
\hline Class & Bacteroidia & Intracellular trafficking, secretion, and vesicular transport & 38.7 & $3.09 \times 10^{-23}$ \\
\hline Class & Mammalia & Carbohydrate transport and metabolism & 59.2 & $3.26 \times 10^{-21}$ \\
\hline Class & Bacteroidia & Lipid transport and metabolism & 28.3 & 0.0466 \\
\hline Class & Bacteroidia & Posttranslational modification, protein turnover, chaperones & 37.5 & 0.027 \\
\hline Class & Bacteroidia & Amino acid transport and metabolism & 50.6 & $1.71 \times 10^{-8}$ \\
\hline Class & Coriobacteriia & Energy production and conversion & 35.7 & $4.37 \times 10^{-6}$ \\
\hline Class & Bacilli & Carbohydrate transport and metabolism & 54.2 & $3.07 \times 10^{-14}$ \\
\hline Class & Mammalia & Defense mechanisms & 87.3 & $1.56 \times 10^{-20}$ \\
\hline Class & Clostridia & Transcription & 38.8 & 0.00493 \\
\hline
\end{tabular}


Table A.2 - continued from previous page

\begin{tabular}{|c|c|c|c|c|}
\hline Rank & LCA & NOG category & RITZ mean (\%) & p-value \\
\hline Class & Deltaproteobacteria & Energy production and conversion & 42 & 0.00531 \\
\hline Class & Clostridia & Inorganic ion transport and metabolism & 20.4 & $2.76 \times 10^{-5}$ \\
\hline Class & Deferribacteres & Energy production and conversion & 31.9 & $1.29 \times 10^{-6}$ \\
\hline Class & Bacteroidia & Translation, ribosomal structure and biogenesis & 32 & $7.75 \times 10^{-8}$ \\
\hline Class & Mammalia & Extracellular structures & 61.5 & $1.33 \times 10^{-16}$ \\
\hline Class & Bacilli & Energy production and conversion & 41.6 & 0.0093 \\
\hline Class & Mammalia & Secondary metabolites biosynthesis, transport and catabolism & 50.9 & 0.00664 \\
\hline Class & Gammaproteobacteria & Carbohydrate transport and metabolism & 34.4 & 0.00085 \\
\hline Class & Actinobacteria & Translation, ribosomal structure and biogenesis & 20 & 0.00066 \\
\hline Class & Gammaproteobacteria & Amino acid transport and metabolism & 32.2 & 0.00219 \\
\hline Class & Clostridia & Cell wall/membrane/envelope biogenesis & 29.2 & 0.0386 \\
\hline Order & Clostridiales & Lipid transport and metabolism & 26.3 & $1.41 \times 10^{-22}$ \\
\hline Order & Bacteroidales & Carbohydrate transport and metabolism & 37.3 & $7.4 \times 10^{-6}$ \\
\hline Order & Rodentia & Intracellular trafficking, secretion, and vesicular transport & 77 & $6.99 \times 10^{-15}$ \\
\hline Order & Clostridiales & Energy production and conversion & 26.9 & $2.12 \times 10^{-94}$ \\
\hline Order & Clostridiales & Carbohydrate transport and metabolism & 35 & $4.36 \times 10^{-144}$ \\
\hline Order & Bacteroidales & Cell wall/membrane/envelope biogenesis & 43.3 & $3.59 \times 10^{-48}$ \\
\hline Order & Clostridiales & Cell motility & 34 & $4.94 \times 10^{-34}$ \\
\hline Order & Rhodobacterales & Amino acid transport and metabolism & 37.2 & 0.00125 \\
\hline Order & Clostridiales & Translation, ribosomal structure and biogenesis & 35 & $3.56 \times 10^{-42}$ \\
\hline Order & Bacteroidales & Inorganic ion transport and metabolism & 42.4 & $1.21 \times 10^{-73}$ \\
\hline Order & Clostridiales & Posttranslational modification, protein turnover, chaperones & 26.2 & $1.55 \times 10^{-22}$ \\
\hline Order & Eggerthellales & Amino acid transport and metabolism & 45.5 & $1.99 \times 10^{-7}$ \\
\hline Order & Clostridiales & Amino acid transport and metabolism & 35.4 & $1.6 \times 10^{-30}$ \\
\hline Order & Clostridiales & Secondary metabolites biosynthesis, transport and catabolism & 35.3 & 0.0155 \\
\hline Order & Rodentia & Inorganic ion transport and metabolism & 63.7 & $7.4 \times 10^{-27}$ \\
\hline Order & Clostridiales & Nucleotide transport and metabolism & 42.9 & $8.8 \times 10^{-9}$ \\
\hline Order & Rodentia & Amino acid transport and metabolism & 53.8 & $2.89 \times 10^{-39}$ \\
\hline Order & Rodentia & Posttranslational modification, protein turnover, chaperones & 56.8 & $2.63 \times 10^{-71}$ \\
\hline Order & Lactobacillales & Translation, ribosomal structure and biogenesis & 49.5 & $2.4 \times 10^{-5}$ \\
\hline Order & Rodentia & Signal transduction mechanisms & 67.2 & $2.19 \times 10^{-44}$ \\
\hline Order & Bacteroidales & Energy production and conversion & 37.3 & $8.85 \times 10^{-19}$ \\
\hline Order & Verrucomicrobiales & Translation, ribosomal structure and biogenesis & 67.2 & $3.14 \times 10^{-6}$ \\
\hline Order & Fusobacteriales & Lipid transport and metabolism & 8.25 & 0.0355 \\
\hline Order & Verrucomicrobiales & Carbohydrate transport and metabolism & 59.1 & $6.67 \times 10^{-6}$ \\
\hline Order & Bacteroidales & Intracellular trafficking, secretion, and vesicular transport & 38.7 & $3.09 \times 10^{-23}$ \\
\hline Order & Rodentia & Carbohydrate transport and metabolism & 63.9 & $4.01 \times 10^{-20}$ \\
\hline Order & Bacteroidales & Lipid transport and metabolism & 28.3 & 0.0466 \\
\hline Order & Bacteroidales & Posttranslational modification, protein turnover, chaperones & 37.5 & 0.027 \\
\hline Order & Bacteroidales & Amino acid transport and metabolism & 50.6 & $1.71 \times 10^{-8}$ \\
\hline Order & Lactobacillales & Carbohydrate transport and metabolism & 58.9 & $1.32 \times 10^{-13}$ \\
\hline $\begin{array}{l}\text { Order } \\
\text { Order }\end{array}$ & $\begin{array}{l}\text { Rodentia } \\
\text { Clostridiales }\end{array}$ & $\begin{array}{l}\text { Defense mechanisms } \\
\text { Transcription }\end{array}$ & $\begin{array}{l}87.3 \\
38.8\end{array}$ & $\begin{array}{l}1.56 \times 10^{-20} \\
0.00493\end{array}$ \\
\hline Order & Clostridiales & Inorganic ion transport and metabolism & 20.4 & $2.76 \times 10^{-5}$ \\
\hline Order & Deferribacterales & Energy production and conversion & 31.9 & $1.29 \times 10^{-6}$ \\
\hline Order & Bacteroidales & Translation, ribosomal structure and biogenesis & 32 & $7.75 \times 10^{-8}$ \\
\hline Order & Rodentia & Extracellular structures & 58 & $1.18 \times 10^{-12}$ \\
\hline Order & Lactobacillales & Energy production and conversion & 41.6 & 0.0093 \\
\hline Order & Rodentia & Secondary metabolites biosynthesis, transport and catabolism & 50.9 & 0.00664 \\
\hline Order & Clostridiales & Cell wall/membrane/envelope biogenesis & 29.2 & 0.0386 \\
\hline Family & Lachnospiraceae & Lipid transport and metabolism & 25.2 & $2.17 \times 10^{-5}$ \\
\hline Family & Bacteroidaceae & Carbohydrate transport and metabolism & 42.2 & 0.019 \\
\hline Family & Lachnospiraceae & Energy production and conversion & 22.5 & $2.73 \times 10^{-31}$ \\
\hline Family & Lachnospiraceae & Carbohydrate transport and metabolism & 32.5 & $1.86 \times 10^{-36}$ \\
\hline Family & Bacteroidaceae & Cell wall/membrane/envelope biogenesis & 41.6 & $4.94 \times 10^{-34}$ \\
\hline Family & Lachnospiraceae & Cell motility & 39.9 & $2.4 \times 10^{-16}$ \\
\hline Family & Rhodobacteraceae & Amino acid transport and metabolism & 37.2 & 0.00125 \\
\hline Family & Bacteroidaceae & Inorganic ion transport and metabolism & 42.6 & $6.93 \times 10^{-43}$ \\
\hline Family & Lachnospiraceae & Posttranslational modification, protein turnover, chaperones & 25.9 & $1.71 \times 10^{-6}$ \\
\hline Family & Eggerthellaceae & Amino acid transport and metabolism & 45.5 & $1.99 \times 10^{-7}$ \\
\hline Family & Porphyromonadaceae & Inorganic ion transport and metabolism & 36.7 & $1.77 \times 10^{-9}$ \\
\hline Family & Clostridiaceae & Carbohydrate transport and metabolism & 40.5 & $8.88 \times 10^{-8}$ \\
\hline Family & Muridae & Inorganic ion transport and metabolism & 64.9 & $8.05 \times 10^{-21}$ \\
\hline Family & Clostridiaceae & Nucleotide transport and metabolism & 54.2 & 0.000202 \\
\hline Family & Muridae & Amino acid transport and metabolism & 54.3 & $1.97 \times 10^{-37}$ \\
\hline Family & Muridae & Posttranslational modification, protein turnover, chaperones & 57 & $6.38 \times 10^{-68}$ \\
\hline Family & Lactobacillaceae & Translation, ribosomal structure and biogenesis & 49.5 & $2.4 \times 10^{-5}$ \\
\hline Family & Muridae & Signal transduction mechanisms & 69.2 & $5.68 \times 10^{-39}$ \\
\hline Family & Eubacteriaceae & Carbohydrate transport and metabolism & 39.2 & $3.46 \times 10^{-5}$ \\
\hline Family & Akkermansiaceae & Translation, ribosomal structure and biogenesis & 67.2 & $3.14 \times 10^{-6}$ \\
\hline Family & Fusobacteriaceae & Lipid transport and metabolism & 8.25 & 0.0355 \\
\hline Family & Oscillospiraceae & Lipid transport and metabolism & 27.5 & $4.87 \times 10^{-5}$ \\
\hline Family & Akkermansiaceae & Carbohydrate transport and metabolism & 59.1 & $6.67 \times 10^{-6}$ \\
\hline Family & Lachnospiraceae & Translation, ribosomal structure and biogenesis & 43.5 & $1.74 \times 10^{-9}$ \\
\hline Family & Lachnospiraceae & Amino acid transport and metabolism & 36.5 & $1.35 \times 10^{-8}$ \\
\hline Family & Muridae & Carbohydrate transport and metabolism & 67.9 & $1.75 \times 10^{-21}$ \\
\hline Family & Clostridiaceae & Translation, ribosomal structure and biogenesis & 41.2 & 0.00846 \\
\hline Family & Bacteroidaceae & Amino acid transport and metabolism & 50.3 & $3.75 \times 10^{-6}$ \\
\hline Family & Oscillospiraceae & Energy production and conversion & 28.8 & $8.16 \times 10^{-6}$ \\
\hline Family & Eubacteriaceae & Cell motility & 20.1 & 0.0269 \\
\hline Family & Lactobacillaceae & Carbohydrate transport and metabolism & 61.5 & $9.2 \times 10^{-14}$ \\
\hline
\end{tabular}


Table A.2 - continued from previous page

\begin{tabular}{|c|c|c|c|c|}
\hline Rank & LCA & NOG category & RITZ mean (\%) & p-value \\
\hline Family & Muridae & Defense mechanisms & 87 & $3.55 \times 10^{-15}$ \\
\hline Family & Oscillospiraceae & Amino acid transport and metabolism & 38.8 & $5.92 \times 10^{-5}$ \\
\hline Family & Oscillospiraceae & Carbohydrate transport and metabolism & 33.5 & $2.42 \times 10^{-21}$ \\
\hline Family & Bacteroidaceae & Intracellular trafficking, secretion, and vesicular transport & 36.4 & $2.2 \times 10^{-15}$ \\
\hline Family & Oscillospiraceae & Cell motility & 38.6 & 0.00142 \\
\hline Family & Bacteroidaceae & Energy production and conversion & 39.1 & $2.66 \times 10^{-14}$ \\
\hline Family & Clostridiaceae & Cell motility & 52 & $1.49 \times 10^{-6}$ \\
\hline Family & Clostridiaceae & Energy production and conversion & 25.1 & 0.000889 \\
\hline Family & Eubacteriaceae & Energy production and conversion & 27.5 & 0.00188 \\
\hline Family & Ruminococcaceae & Amino acid transport and metabolism & 42.7 & 0.0233 \\
\hline Family & Ruminococcaceae & Carbohydrate transport and metabolism & 47.3 & $1.51 \times 10^{-6}$ \\
\hline Family & Eubacteriaceae & Translation, ribosomal structure and biogenesis & 33.9 & 0.00235 \\
\hline Family & Deferribacteraceae & Energy production and conversion & 31.9 & $1.29 \times 10^{-6}$ \\
\hline Family & Clostridiaceae & Posttranslational modification, protein turnover, chaperones & 27.1 & 0.0224 \\
\hline Family & Muridae & Extracellular structures & 58 & $1.18 \times 10^{-12}$ \\
\hline Family & Porphyromonadaceae & Cell wall/membrane/envelope biogenesis & 48.1 & $8.97 \times 10^{-12}$ \\
\hline Family & Porphyromonadaceae & Energy production and conversion & 37.3 & 0.00267 \\
\hline Family & Lactobacillaceae & Energy production and conversion & 41.6 & 0.0093 \\
\hline Family & Muridae & Secondary metabolites biosynthesis, transport and catabolism & 50.9 & 0.00664 \\
\hline Family & Bacteroidaceae & Translation, ribosomal structure and biogenesis & 31.8 & 0.000105 \\
\hline Family & Oscillospiraceae & Inorganic ion transport and metabolism & 25 & 0.00567 \\
\hline Family & Muridae & Intracellular trafficking, secretion, and vesicular transport & 82.1 & $1.96 \times 10^{-11}$ \\
\hline Genus & Bacteroides & Carbohydrate transport and metabolism & 42.2 & 0.019 \\
\hline Genus & Bacteroides & Cell wall/membrane/envelope biogenesis & 41.6 & $4.94 \times 10^{-34}$ \\
\hline Genus & Bacteroides & Inorganic ion transport and metabolism & 42.6 & $6.93 \times 10^{-43}$ \\
\hline Genus & Parabacteroides & Inorganic ion transport and metabolism & 36.7 & $1.77 \times 10^{-9}$ \\
\hline Genus & Clostridium & Carbohydrate transport and metabolism & 40.7 & $3.63 \times 10^{-7}$ \\
\hline Genus & Mus & Inorganic ion transport and metabolism & 64.2 & $2.84 \times 10^{-20}$ \\
\hline Genus & Mus & Amino acid transport and metabolism & 55.9 & $1.89 \times 10^{-32}$ \\
\hline Genus & Mus & Posttranslational modification, protein turnover, chaperones & 55.1 & $5.92 \times 10^{-56}$ \\
\hline Genus & Lactobacillus & Translation, ribosomal structure and biogenesis & 47.2 & 0.000274 \\
\hline Genus & Mus & Signal transduction mechanisms & 70.7 & $3.52 \times 10^{-33}$ \\
\hline Genus & Roseburia & Cell motility & 47.2 & 0.000777 \\
\hline Genus & Eubacterium & Carbohydrate transport and metabolism & 39.2 & $3.46 \times 10^{-5}$ \\
\hline Genus & Akkermansia & Translation, ribosomal structure and biogenesis & 67.2 & $3.14 \times 10^{-6}$ \\
\hline Genus & Oscillibacter & Lipid transport and metabolism & 27.5 & $4.87 \times 10^{-5}$ \\
\hline Genus & Akkermansia & Carbohydrate transport and metabolism & 59.1 & $6.67 \times 10^{-6}$ \\
\hline Genus & Lachnoclostridium & Carbohydrate transport and metabolism & 16 & 0.00128 \\
\hline Genus & Dorea & Carbohydrate transport and metabolism & 32.9 & 0.000657 \\
\hline Genus & Dorea & Energy production and conversion & 38 & $2.98 \times 10^{-7}$ \\
\hline Genus & Mus & Carbohydrate transport and metabolism & 66.6 & $2.05 \times 10^{-17}$ \\
\hline Genus & Bacteroides & Amino acid transport and metabolism & 50.3 & $3.75 \times 10^{-6}$ \\
\hline Genus & Oscillibacter & Energy production and conversion & 28.8 & $8.16 \times 10^{-6}$ \\
\hline Genus & Eubacterium & Cell motility & 20.1 & 0.0269 \\
\hline Genus & Lactobacillus & Carbohydrate transport and metabolism & 61.5 & $9.2 \times 10^{-14}$ \\
\hline Genus & Mus & Defense mechanisms & 87 & $3.55 \times 10^{-15}$ \\
\hline Genus & Oscillibacter & Amino acid transport and metabolism & 38.8 & $5.92 \times 10^{-5}$ \\
\hline Genus & Oscillibacter & Carbohydrate transport and metabolism & 33.5 & $2.42 \times 10^{-21}$ \\
\hline Genus & Bacteroides & Intracellular trafficking, secretion, and vesicular transport & 36.4 & $2.2 \times 10^{-15}$ \\
\hline Genus & Oscillibacter & Cell motility & 38.6 & 0.00142 \\
\hline Genus & Bacteroides & Energy production and conversion & 39.1 & $2.66 \times 10^{-14}$ \\
\hline Genus & Clostridium & Cell motility & 46.4 & $3.56 \times 10^{-7}$ \\
\hline Genus & Clostridium & Energy production and conversion & 21.8 & 0.00125 \\
\hline Genus & Eubacterium & Energy production and conversion & 22.8 & 0.000397 \\
\hline Genus & Flavonifractor & Energy production and conversion & 31.8 & 0.00938 \\
\hline Genus & Subdoligranulum & Carbohydrate transport and metabolism & 41 & 0.00629 \\
\hline Genus & Eubacterium & Translation, ribosomal structure and biogenesis & 33.9 & 0.00235 \\
\hline Genus & Mucispirillum & Energy production and conversion & 31.9 & $1.29 \times 10^{-6}$ \\
\hline Genus & Roseburia & Carbohydrate transport and metabolism & 27.4 & 0.00353 \\
\hline Genus & Dorea & Amino acid transport and metabolism & 34 & 0.00568 \\
\hline Genus & Parabacteroides & Cell wall/membrane/envelope biogenesis & 48.1 & $8.97 \times 10^{-12}$ \\
\hline Genus & Anaerotruncus & Carbohydrate transport and metabolism & 36.2 & $8.74 \times 10^{-5}$ \\
\hline Genus & Lactobacillus & Energy production and conversion & 41.6 & 0.0093 \\
\hline Genus & Mus & Secondary metabolites biosynthesis, transport and catabolism & 50.9 & 0.00664 \\
\hline Genus & Bacteroides & Translation, ribosomal structure and biogenesis & 31.8 & 0.000105 \\
\hline Genus & Enterorhabdus & Amino acid transport and metabolism & 51.2 & 0.0476 \\
\hline Genus & Ruminococcus & Carbohydrate transport and metabolism & 67.1 & 0.0106 \\
\hline Genus & Oscillibacter & Inorganic ion transport and metabolism & 25 & 0.00567 \\
\hline Genus & Mus & Intracellular trafficking, secretion, and vesicular transport & 82.1 & $1.96 \times 10^{-11}$ \\
\hline Genus & Butyrivibrio & Carbohydrate transport and metabolism & 26.4 & 0.00423 \\
\hline Genus & Mus & Extracellular structures & 50.3 & $2.55 \times 10^{-7}$ \\
\hline Genus & Dorea & Posttranslational modification, protein turnover, chaperones & 33.5 & 0.04 \\
\hline Species & Lachnospiraceae bacterium A4 & Carbohydrate transport and metabolism & 31.7 & $5.56 \times 10^{-6}$ \\
\hline Species & Lachnospiraceae bacterium 28-4 & Cell motility & 40.7 & 0.0036 \\
\hline Species & Lachnospiraceae bacterium COE1 & Carbohydrate transport and metabolism & 34.8 & 0.00099 \\
\hline Species & Parabacteroides goldsteinii & Inorganic ion transport and metabolism & 37.7 & $1.01 \times 10^{-8}$ \\
\hline Species & Clostridium sp. ASF502 & Carbohydrate transport and metabolism & 50.6 & 0.000129 \\
\hline Species & Lachnospiraceae bacterium $28-4$ & Energy production and conversion & 20.8 & $7.7 \times 10^{-10}$ \\
\hline Species & Mus musculus & Inorganic ion transport and metabolism & 64.2 & $2.84 \times 10^{-20}$ \\
\hline Species & Mus musculus & Amino acid transport and metabolism & 55.9 & $1.89 \times 10^{-32}$ \\
\hline Species & Mus musculus & Posttranslational modification, protein turnover, chaperones & 55.1 & $5.92 \times 10^{-56}$ \\
\hline Species & Lachnospiraceae bacterium 3-2 & Carbohydrate transport and metabolism & 35.4 & 0.00664 \\
\hline Species & Mus musculus & Signal transduction mechanisms & 70.7 & $3.52 \times 10^{-33}$ \\
\hline
\end{tabular}


Table A.2 - continued from previous page

\begin{tabular}{|c|c|c|c|c|}
\hline Rank & LCA & NOG category & RITZ mean (\%) & p-value \\
\hline Species & Oscillibacter sp. $1-3$ & Lipid transport and metabolism & 34.1 & 0.000157 \\
\hline Species & Akkermansia muciniphila & Carbohydrate transport and metabolism & 59 & $3.97 \times 10^{-5}$ \\
\hline Species & Lachnospiraceae bacterium M18-1 & Translation, ribosomal structure and biogenesis & 46.6 & 0.00664 \\
\hline Species & Dorea sp. 5-2 & Carbohydrate transport and metabolism & 32.2 & 0.0246 \\
\hline Species & Lachnospiraceae bacterium A4 & Amino acid transport and metabolism & 38.8 & 0.000523 \\
\hline Species & Mus musculus & Carbohydrate transport and metabolism & 66.6 & $2.05 \times 10^{-17}$ \\
\hline Species & Lachnospiraceae bacterium A4 & Lipid transport and metabolism & 8.05 & 0.0463 \\
\hline Species & Lachnospiraceae bacterium M18-1 & Cell motility & 38.3 & 0.000453 \\
\hline Species & Eubacterium plexicaudatum & Cell motility & 20.1 & 0.0269 \\
\hline Species & Lachnospiraceae bacterium A2 & Carbohydrate transport and metabolism & 45.1 & 0.000758 \\
\hline Species & Lachnospiraceae bacterium 28-4 & Carbohydrate transport and metabolism & 41.9 & $2.14 \times 10^{-7}$ \\
\hline Species & Eubacterium plexicaudatum & Carbohydrate transport and metabolism & 40.7 & 0.0014 \\
\hline Species & Mus musculus & Defense mechanisms & 85.6 & $4.35 \times 10^{-12}$ \\
\hline Species & Firmicutes bacterium ASF500 & Amino acid transport and metabolism & 37.9 & 0.00362 \\
\hline Species & Lachnospiraceae bacterium A4 & Energy production and conversion & 21.7 & 0.00117 \\
\hline Species & Firmicutes bacterium ASF500 & Cell motility & 39.7 & 0.0259 \\
\hline Species & Oscillibacter sp. 1-3 & Cell motility & 38.6 & 0.00142 \\
\hline Species & Bacteroides vulgatus & Cell wall/membrane/envelope biogenesis & 53.5 & $7.01 \times 10^{-22}$ \\
\hline Species & Firmicutes bacterium ASF500 & Carbohydrate transport and metabolism & 44.8 & $1.58 \times 10^{-9}$ \\
\hline Species & Lachnospiraceae bacterium 10-1 & Energy production and conversion & 14.5 & $2.41 \times 10^{-6}$ \\
\hline Species & Oscillibacter sp. $1-3$ & Carbohydrate transport and metabolism & 34.6 & $3.99 \times 10^{-19}$ \\
\hline Species & Oscillibacter sp. 1-3 & Energy production and conversion & 34.5 & 0.000193 \\
\hline Species & Flavonifractor plautii & Energy production and conversion & 31.8 & 0.00938 \\
\hline Species & Subdoligranulum variabile & Carbohydrate transport and metabolism & 41 & 0.00629 \\
\hline Species & Mucispirillum schaedleri & Energy production and conversion & 31.9 & $1.29 \times 10^{-6}$ \\
\hline Species & Lachnospiraceae bacterium A4 & Cell motility & 46.9 & 0.00114 \\
\hline Species & Firmicutes bacterium ASF500 & Energy production and conversion & 56.5 & 0.0246 \\
\hline Species & Dorea sp. 5-2 & Amino acid transport and metabolism & 31.8 & 0.0144 \\
\hline Species & Parabacteroides goldsteinii & Cell wall/membrane/envelope biogenesis & 44.8 & $2.95 \times 10^{-6}$ \\
\hline Species & Anaerotruncus sp. G3(2012) & Carbohydrate transport and metabolism & 32.8 & 0.012 \\
\hline Species & Mus musculus & Secondary metabolites biosynthesis, transport and catabolism & 50.9 & 0.00664 \\
\hline Species & Enterorhabdus caecimuris & Amino acid transport and metabolism & 51.2 & 0.0476 \\
\hline Species & Oscillibacter valericigenes & Amino acid transport and metabolism & 32.3 & 0.00166 \\
\hline Species & Oscillibacter sp. 1-3 & Inorganic ion transport and metabolism & 25 & 0.00567 \\
\hline Species & Mus musculus & Intracellular trafficking, secretion, and vesicular transport & 82.1 & $1.96 \times 10^{-11}$ \\
\hline Species & Lachnospiraceae bacterium 10-1 & Cell motility & 34.7 & 0.00155 \\
\hline Species & Lachnospiraceae bacterium A4 & Translation, ribosomal structure and biogenesis & 38.1 & 0.00813 \\
\hline Species & Eubacterium plexicaudatum & Energy production and conversion & 23.4 & 0.0266 \\
\hline Species & Lachnospiraceae bacterium $10-1$ & Carbohydrate transport and metabolism & 32.7 & $5.64 \times 10^{-6}$ \\
\hline Species & Firmicutes bacterium ASF500 & Lipid transport and metabolism & 33.6 & 0.0135 \\
\hline Species & Dorea sp. $5-2$ & Energy production and conversion & 37.5 & 0.0147 \\
\hline Species & Dorea sp. 5-2 & Posttranslational modification, protein turnover, chaperones & 33.5 & 0.04 \\
\hline
\end{tabular}


Table A.3: Hygeometric tests for over-representation of taxa in RIA hierarchical clusters

\begin{tabular}{llll}
\hline \multicolumn{1}{c}{ name } & group & \multicolumn{1}{c}{ RIAC1 } & RIAC2 \\
\hline Bacteroidaceae & Family & $2.29 \times 10^{-11}$ & \\
Bacteroidales & Order & $2.34 \times 10^{-25}$ & \\
Bacteroides & Genus & $2.29 \times 10^{-11}$ & \\
Bacteroides vulgatus & Species & 0.0223 & \\
Bacteroidetes & Phylum & $1.63 \times 10^{-26}$ & \\
Bacteroidia & Class & $2.34 \times 10^{-25}$ & \\
Chordata & Phylum & 0.00658 & \\
Clostridia & Class & & 0.0164 \\
Clostridiales & Order & & 0.0178 \\
Firmicutes & Phylum & & $1.17 \times 10^{-7}$ \\
Lachnospiraceae & Family & & 0.039 \\
Mammalia & Class & 0.0118 & \\
Muridae & Family & 0.006 & \\
Mus & Genus & 0.00465 & \\
Mus musculus & Species & 0.00232 & \\
Parabacteroides & Genus & $2.63 \times 10^{-9}$ & \\
Parabacteroides goldsteinii & Species & $2.46 \times 10^{-9}$ & \\
Porphyromonadaceae & Family & $4.75 \times 10^{-9}$ & \\
Rodentia & Order & 0.0356 & \\
\hline
\end{tabular}


Table A.4: Hygeometric tests for over-representation of taxa in RITZ hierarchical clusters

\begin{tabular}{|c|c|c|c|c|c|}
\hline name & group & RITZC1 & RITZC2 & RITZC3 & RITZC4 \\
\hline Akkermansia & Genus & & & 0.0029 & \\
\hline $\begin{array}{l}\text { Akkermansia } \\
\text { muciniphila }\end{array}$ & Species & & & 0.0125 & \\
\hline Akkermansiaceae & Family & & & 0.0029 & \\
\hline Bacteroidaceae & Family & & 0.00516 & 0.00213 & \\
\hline Bacteroidales & Order & & & $2.21 \times 10^{-18}$ & \\
\hline Bacteroides & Genus & & 0.00516 & 0.00213 & \\
\hline $\begin{array}{l}\text { Bacteroides } \\
\text { vulgatus }\end{array}$ & Species & & & 0.0012 & \\
\hline Bacteroidetes & Phylum & & & $2.7 \times 10^{-18}$ & \\
\hline Bacteroidia & Class & & & $2.21 \times 10^{-18}$ & \\
\hline Chordata & Phylum & & & $1.78 \times 10^{-13}$ & $4.32 \times 10^{-49}$ \\
\hline Clostridia & Class & 0.0188 & & & \\
\hline Clostridiales & Order & 0.0172 & & & \\
\hline Clostridium & Genus & & & & 0.0363 \\
\hline $\begin{array}{l}\text { Clostridium sp. } \\
\text { ASF502 }\end{array}$ & Species & & & & 0.0227 \\
\hline Coriobacteriia & Class & & 0.0308 & & \\
\hline Firmicutes & Phylum & $2.62 \times 10^{-5}$ & 0.0398 & & \\
\hline Lachnospiraceae & Family & 0.00085 & & & \\
\hline $\begin{array}{l}\text { Lachnospiraceae } \\
\text { bacterium } 10-1\end{array}$ & Species & 0.0156 & & & \\
\hline Mammalia & Class & & & $5.64 \times 10^{-11}$ & $1.22 \times 10^{-48}$ \\
\hline Muridae & Family & & & $2.05 \times 10^{-9}$ & $1.76 \times 10^{-32}$ \\
\hline Mus & Genus & & & $4.1 \times 10^{-8}$ & $4.25 \times 10^{-27}$ \\
\hline Mus musculus & Species & & & $1.31 \times 10^{-7}$ & $1.71 \times 10^{-26}$ \\
\hline Oscillibacter & Genus & & 0.00327 & & \\
\hline $\begin{array}{l}\text { Oscillibacter sp. } \\
1-3\end{array}$ & Species & & $2.19 \times 10^{-5}$ & & \\
\hline Oscillospiraceae & Family & & 0.00327 & & \\
\hline Parabacteroides & Genus & & & $4.18 \times 10^{-21}$ & \\
\hline $\begin{array}{l}\text { Parabacteroides } \\
\text { goldsteinii }\end{array}$ & Species & & & $2.13 \times 10^{-19}$ & \\
\hline \multicolumn{4}{|c|}{ Porphyromonadace amily } & $1.18 \times 10^{-20}$ & \\
\hline Rodentia & Order & & & $5.09 \times 10^{-10}$ & $3 \times 10^{-35}$ \\
\hline Verrucomicrobia & Phylum & & & 0.0029 & \\
\hline Verrucomicrobiae & Class & & & 0.0029 & \\
\hline Verrucomicrobiale & sOrder & & & 0.0029 & \\
\hline
\end{tabular}


Curriculum Vitae 


\section{AREAS OF EXPERTISE}

Reporting skills

Academic research

Cell and molecular techniques

Synthesis and separation

techniques.

Analytical techniques

Bioinformatics

Statistics

Software Development

C++

$R$ language

PROFESSIONAL

Bachelor's degree in

Biochemistry

PERSONAL SKILLS

Initiative

Highly competent

Efficient with computers

\section{Patrick Smyth}

\section{Resume}

\author{
PERSONAL SUMMARY
}

I have excellent research potential and an ability to actively contribute to the research project's goals as well as a good standard of written English and French. I can interact with all researchers in a constructive, creative and professional manner.

I possess expertise in cell and molecular techniques, synthesis and separation techniques, analytical techniques, biostatistics/informatics, software development, and metaproteomics.

PROFESSIONAL EXPERIENCE

\section{University of Ottawa}

MASTERS THESIS September 2018 - December 2020

Developed a software to identify and analyze partially labeled peptides for microbiome dynamics. My research led to a publication in the journal of analytical chemistry (DOI: 10.1021/acs.analchem.0c02070). I was supervised by Dr. Daniel Figeys.

Duties:

- Building and testing software using $\mathrm{R}$ and $\mathrm{C}++$.

- Performing the most current and effective methods in bioinformatics and biostatistics for analysis of big data.

- Analyzing gut microbiome data.

- Making presentable figures about research findings.

- Frequent participation in conferences and professional workshops.

- Searching and summarizing literature related to research.

- Producing a final thesis report and presenting the research findings to lab colleagues and committee members.

\section{University of Ottawa}

TECHNOMISE PROGRAM January 2018 - December 2020

I participated in various professional development workshops and seminars related to gut microbiome research as part of the TECHNOMISE program. Such workshops include an omics bootcamp and classes on project management, team building, and communication skills.

\section{ASMS}

Conference presentation July 2020

Presented a 10-minute talk related to my research at the American Society for Mass Spectrometry (ASMS) conference.

ISMB

Poster presentation June 2020

Presented a poster and an 8-minute short talk at ISMB 2020 


\section{KEY SKILLS AND COMPETENCIES}

\section{analytical chemistry}

- Knowledgeable in the study of activity, chemical equilibrium and titration curves on complexation and neutralization systems.

- Treatment of analytical data.

AREAS OF EXPERTISE

Reporting skills

Academic research

Cell and molecular techniques

Synthesis and separation

techniques.

Analytical techniques

Bioinformatics

Statistics

Software Development

C++

$R$ language

PROFESSIONAL

Bachelor's degree

PERSONAL SKILLS

Initiative

Highly competent

Efficient with computers
- Applying methods for precipitation and solubility product.

\section{Spectroscopy}

- Knowledgeable in the principles of UV-vis-, IR-, NMR-, CDspectroscopy, and mass spectrometry, as well as their spectral analysis.

\section{Chemistry}

- Knowledgeable in various synthesis techniques

- Liquid and gas chromatography, including other separation and purification techniques.

\section{Cell and molecular biology}

- Knowledgeable in the amplification and preparation of DNA constructs.

- Expression and purification of desired proteins.

- Separation of protein, DNA, and RNA.

- Cell culture techniques.

\section{Bioinformatics}

- Knowledgeable in algorithms for the classification and analysis of big data.

- Machine Learning and AI

- Metaproteomics

- Software Development.

- Biostatistics (Particularly in Bayesian inference)

ACADEMIC QUALIFICATIONS

BSc (Hons) Biochemistry

Concordia University 2013 - 2018 